\title{
Exposing the dark sector with future $Z$ factories
}

\author{
Jia Liu, ${ }^{1,2, \dagger}$ Lian-Tao Wang, ${ }^{1,3,4, \dagger}$ Xiao-Ping Wang, ${ }^{5,2, *}$ and Wei Xue ${ }^{6,7, \S}$ \\ ${ }^{1}$ Enrico Fermi Institute, The University of Chicago, 5640 S Ellis Avenue, Chicago, Illinois 60637, USA \\ ${ }^{2}$ PRISMA Cluster of Excellence and Mainz Institute for Theoretical Physics, \\ Johannes Gutenberg University, 55099 Mainz, Germany \\ ${ }^{3}$ Department of Physics, The University of Chicago, 5640 S Ellis Avenue, Chicago, Illinois 60637, USA \\ ${ }^{4}$ Kavli Institute for Cosmological Physics, The University of Chicago, \\ 5640 S Ellis Avenue, Chicago, Illinois 60637, USA \\ ${ }^{5}$ High Energy Physics Division, Argonne National Laboratory, Argonne, Illinois 60439 \\ ${ }^{6}$ Theoretical Physics Department, CERN, CH-1211 Geneva 23, Switzerland \\ ${ }^{7}$ Center for Theoretical Physics, Massachusetts Institute of Technology, \\ Cambridge, Massachusetts 02139, USA
}

(Received 9 January 2018; revised manuscript received 20 April 2018; published 31 May 2018)

\begin{abstract}
We investigate the prospects of searching dark sector models via exotic $Z$-boson decay at future $e^{+} e^{-}$ colliders with Giga $Z$ and Tera $Z$ options. Four general categories of dark sector models, Higgs portal dark matter, vector-portal dark matter, inelastic dark matter, and axionlike particles, are considered. Focusing on channels motivated by the dark sector models, we carry out a model-independent study of the sensitivities of $Z$ factories in probing exotic decays. The limits on branching ratios of the exotic $Z$ decay are typically $\mathcal{O}\left(10^{-6}-10^{-8.5}\right)$ for the Giga $Z$ and $\mathcal{O}\left(10^{-7.5}-10^{-11}\right)$ for the Tera $Z$, and they are compared with the projection for the high luminosity LHC. We demonstrate that future $Z$ factories can provide its unique and leading sensitivity and highlight the complementarity with other experiments, including the indirect and direct dark matter search limits and the existing collider limits. Future $Z$ factories will play a leading role in uncovering the hidden sector of the Universe in the future.
\end{abstract}

DOI: 10.1103/PhysRevD.97.095044

\section{INTRODUCTION}

Searching for dark sector particles, including dark matter (DM) itself and other associated states, is a central goal of many experimental programs around the world. In the mass range between $\mathrm{MeV}$ and $\mathrm{TeV}$, collider searches remain a crucial method to look for these hidden particles. Since the dark sector particles typically only have weak couplings with the Standard Model, colliders with higher luminosity are natural places to lead this quest. Recently, there have been a couple of proposals for future $Z$ factories based on circular $e^{+} e^{-}$colliders, including FCC-ee and CEPC [1-4], which are considering both Giga- $Z$ and Tera- $Z$ options. Giga $Z$ (Tera $Z$ ) means running the electron collider at $Z$-pole energy and accumulating $10^{9}\left(10^{12}\right) Z$ 's. Given the

\footnotetext{
*Corresponding author. xia.wang@anl.gov

†iuj1@uchicago.edu

*liantaow@uchicago.edu

§weixue@mit.edu

Published by the American Physical Society under the terms of the Creative Commons Attribution 4.0 International license. Further distribution of this work must maintain attribution to the author(s) and the published article's title, journal citation, and DOI. Funded by SCOAP.
}

measured cross section of hadronic $Z$ is $30.5 \mathrm{nb}$ [5], the integrated luminosity for Giga $Z\left(10^{9} Z\right)$ and Tera $Z\left(10^{12} Z\right.$ in the plan of FCC-ee) are $22.9 \mathrm{fb}^{-1}$ and $22.9 \mathrm{ab}^{-1}$, respectively. In this paper, we give projections on the sensitivities of $Z$-factory searches to a set of $Z$ rare decay channels inspired by the dark sector models.

A coupling between $Z$ and dark sector states, dubbed as a "portal," is quite generic in dark sector models. We can classify the portals based on the type of operators through which they are implemented, as follows (for recent reviews, see Refs. [6-8]):

(i) Marginal operators: Higgs portal [9-16] and vectorportal DM models [17-22], in which the dark sector interacts with the $Z$ boson via SM Higgs mixing or gauge boson mixing. The signal is exotic $Z$ decay into SM final states with missing energy.

(ii) Dimension-5 operators: Axionlike particles (ALPs) [23-35], with anomalous coupling to a $Z$ boson and photon. The signal is exotic $Z$ decay into ALPs and a photon.

(iii) Higher-dimensional operators: Magnetic inelastic DM and Rayleigh DM models [36-40], in which the dark sector interacts with $Z$ via a magnetic dipole or Rayleigh operator. The signal is exotic $Z$ decay into a photon and missing energy. 
In addition to using exotic decay measurements to probe these models, we also compare the reach with direct and indirect dark matter detection experiments, current limits from collider searches, and estimated sensitivities of the high luminosity run of the LHC (HL-LHC). Our results demonstrate that the $Z$-factory measurement will provide the leading sensitivities in many cases. We also include thermal relic abundance, with the understanding that it should serve as an interesting benchmark point, rather than a strict limit.

There have been previous works on constraining the dark sector related new physics from $Z$ properties at future $e^{+} e^{-}$ colliders, including a dark photon [41,42]; sterile neutrino model [43,44]; $Z$ invisible width, e.g., Ref. [45]; rare SM $Z$ decays [46-50]; and light $C P$-odd Higgs bosons and supersymmetric models [51-55]. Recently, this topic has been addressed for some specific models [56-60]. Large Electron-Positron collider (LEP) has also searched for exotic $Z$ decays into light Higgs [61,62], two light Higgs in the minimal supersymmetric Standard Model [63], photon and missing energy [64-66], and three photons [67]. There are also direct searches for DM particles at LEP-II via mono photon final states [68].

In Sec. II, we briefly outline the DM indirect and direct searches and the DM relic abundance, in order to compare with the Z-decay searches in Sec. III. Section III focuses on well-defined and representative dark sector models to illustrate the power of exotic $Z$-decay search at a $Z$ factory. Certainly, we cannot cover all the dark sector models for exotic $Z$ decay. Therefore, we list the possible topologies for exotic $Z$ decay according to the final states and number of resonances in Sec. IV. For each decay topology, we comment on the origin of possible UV models, provide the appropriate cuts for each topology, and present the sensitivity on the exotic Z-decay branching ratio (BR). In Sec. V, we conclude.

\section{DM RELIC ABUNDANCE AND INDIRECT AND DIRECT DETECTION}

In this section, we briefly describe the inputs from DM direct detection, indirect detection, and relic abundance employed in this study.

DM direct detection experiments look for DM collision with nuclei in the detector, which leaves visible energy in terms of the phonon, electron, and photon signals. We are interested in the kind of collision which provides a spinindependent cross section with nuclei, where Xenon type experiments such as XENON1T [69], LUX [70], and PANDAX-II [71] provide the best sensitivity for large DM mass. For small DM mass, e.g., $<5 \mathrm{GeV}$, CRESST-II [72] and CDMSlite [73] provide better sensitivity; because their nuclei are lighter than Xenon, they can obtain more energy transferred from light DM collisions.

DM indirect detection experiments search for DM annihilation products like photons, electrons, positrons, and antiprotons from astrophysical sources. We consider the gamma-ray line searches by Fermi-LAT [74], continuous gamma-ray limits from dwarf galaxies [75], and $e^{ \pm}$flux measurements from AMS-02 [76]. And we consider constraints from the cosmic microwave background (CMB), where DM annihilation products heat and ionize the plasma during the recombination epoch [77]. When the DM annihilation cross section is proportional to the DM velocity square $v^{2}$, dubbed the "p-wave cross section," the constraints from the indirect detection are negligible. If DM annihilates into two photons, the leading constraints from indirect detection normally are the gamma-ray line and CMB searches.

The relic abundance $\Omega h^{2}=0.12$ from the Planck Collaboration [77] is used in this paper as a benchmark point. We assume a standard thermal freeze-out. Therefore, the relic abundance only depends on the thermal average of the DM annihilation cross section $\sigma v{ }^{1}$

\section{HIDDEN SECTOR MODELS AND EXOTIC $Z$ DECAYS}

In this section, we discuss several classes of wellmotivated dark sector models, such as Higgs portal DM, vector-portal DM, inelastic DM, and ALPs. These models can be probed by the exotic $Z$ decays in future $e^{+} e^{-} Z$ factories. It is a demonstration of the capability of the $e^{+} e^{-}$ collider as a new physics search machine and a novel intensity frontier experiment.

For each model, we point out how it could be probed by the exotic $Z$ decays. The existing limits from cosmology, astrophysics, and colliders are presented and compared with the reach of the $Z$ factories. If the model contains a dark matter candidate, we will derive the DM relic density by assuming thermal production. The limits from exotic $Z$ decay are obtained from the general analysis presented in detail in Sec. IV. A.

\section{A. Higgs portal fermionic DM}

The Higgs portal is a particular simple possibility to extend the Standard Model and link it with hidden sectors. After discovering the Higgs, searching for another fundamental scalar will help us improve our understanding of electroweak symmetry breaking. Interestingly, the other scalar is potentially related to some enigma in cosmology, such as baryogenesis and DM. Here, we will study the discovery potential of this scalar and its hidden sector by using the exotic $Z$ decays from a future $e^{+} e^{-}$collider.

\section{Model}

We start with a fermionic DM, $\chi$, interacting with a singlet real scalar $S$. $S$ couples to SM via the Higgs portal, and $\mathrm{DM} \chi$ is stable due to the $U(1)_{\chi}$ symmetry $[15,78-84]$.

\footnotetext{
${ }^{1}$ As a caveat, this choice relies on the assumption of the standard thermal freeze-out. Some nonthermal process or other interesting model building of the hidden sector for $\mathrm{GeV}$ DM can give us a different dark matter relic density predication, which is not the focus of this paper.
} 
The general Lagrangian of the simplified model is written down as follows [15]:

$$
\begin{aligned}
\mathcal{L}= & \frac{1}{2} \partial_{\mu} S \partial^{\mu} S-\frac{\mu_{S}^{2}}{2} S^{2}-\frac{\lambda_{3}}{6} S^{3}-\frac{\lambda_{4}}{24} S^{4}-\lambda_{1}\left(H^{\dagger} H\right) S \\
& -\lambda_{2}\left(H^{\dagger} H\right) S^{2}+\bar{\chi}\left(i \not-m_{\chi}^{0}\right) \chi-y_{\chi} S \bar{\chi} \chi+\left|D_{\mu} H\right|^{2} \\
& -\mu_{H}^{2}\left(H^{\dagger} H\right)-\lambda_{H}\left(H^{\dagger} H\right)^{2} .
\end{aligned}
$$

We assume $\mu_{H}^{2}<0$ and $\mu_{S}^{2}<0$, which trigger spontaneous symmetry breaking of the SM and hidden sector. The treelevel vacuum stability condition requires $\lambda_{H}>0, \lambda_{4}>0$; and if $\lambda_{2}<0,\left|\lambda_{2}\right|>\sqrt{\lambda_{H} \lambda_{4} / 24}$ should be satisfied. In the broken phase, the Higgs and the singlet scalar obtain their vacuum expectation values (vevs) $v_{H}$ and $v_{S}$, respectively,

$$
H=\frac{1}{\sqrt{2}}\left(v_{H}+h\right), \quad S=v_{S}+s .
$$

Accordingly, the DM mass $m_{\chi}^{0}$ is shifted to $m_{\chi}=m_{\chi}^{0}+y_{\chi} v_{S}$, which is treated as a free parameter here. Adding the extrema condition that $\partial_{s} V=0$ and $\partial_{h} V=0$, where $V$ is the scalar potential, we will have the mass matrix of $s$ and $h$,

$$
\begin{aligned}
& M_{11}^{2}=2 \lambda_{H} v_{H}^{2}, \\
& M_{12}^{2}=M_{21}^{2}=\left(\lambda_{1}+2 \lambda_{2} v_{S}\right) v_{H}, \\
& M_{22}^{2}=-\frac{\lambda_{1} v_{H}^{2}}{2 v_{S}}+\frac{\lambda_{3} v_{S}}{2}+\frac{\lambda_{4} v_{S}^{2}}{3} .
\end{aligned}
$$

The scalar mass eigenstates $\tilde{h}$ and $\tilde{s}$ are obtained via the following rotation,

$$
\left(\begin{array}{l}
\tilde{h} \\
\tilde{s}
\end{array}\right)=\left(\begin{array}{cc}
\cos \alpha & -\sin \alpha \\
\sin \alpha & \cos \alpha
\end{array}\right)\left(\begin{array}{l}
h \\
s
\end{array}\right),
$$

where

$$
\tan (2 \alpha)=\frac{2 M_{12}^{2}}{M_{22}^{2}-M_{11}^{2}} .
$$

The masses of $\tilde{h}$ and $\tilde{s}$ are

$m_{\tilde{h}, \tilde{s}}^{2}=\frac{1}{2}\left(M_{11}^{2}+M_{22}^{2} \pm \sqrt{\left(M_{11}^{2}-M_{22}^{2}\right)^{2}+4\left(M_{12}^{2}\right)^{2}}\right)$.

Let us pause here to count the relevant free parameters for the scalars. There are nine parameters including $\mu_{S}, \mu_{H}$, $\lambda_{1,2,3,4}, \lambda_{H}$, and two vevs $v_{H}$ and $v_{S}$. The extrema conditions eliminate two of them: $\mu_{S}$ and $\mu_{H}$. By changing to the mass eigenstate basis, the five physical observables are $m_{\tilde{h}}, m_{\tilde{s}}$, $v_{H}, v_{S}$, and mixing angle $\sin \alpha$, which are determined by seven parameters. Without losing generality, we set the coefficients $\lambda_{1}$ and $\lambda_{3}$ appearing in odd terms of $S$ to be 0 , which can be achieved by adding some additional quantum number or $Z_{2}$ symmetry for $S$. Having observed that the Higgs mass $m_{\tilde{h}}=125 \mathrm{GeV}$ and $v_{H}=246 \mathrm{GeV}$, this leads to three final free parameters $m_{\tilde{s}}, v_{S}$, and $\sin \alpha$.

The decay rates and branching ratios relevant to the scalar searches are presented below. In the case that $m_{\tilde{h}}>2 m_{\tilde{s}}$, the SM Higgs decays to two $\tilde{s}$ with decay width

$$
\begin{aligned}
\Gamma(\tilde{h} \rightarrow \tilde{s} \tilde{s})= & \frac{\sin ^{2} \alpha \cos ^{2} \alpha}{32 \pi} \sqrt{1-\frac{4 m_{\tilde{s}}^{2}}{m_{\tilde{h}}^{2}}}\left(1+2 \frac{m_{\tilde{s}}^{2}}{m_{\tilde{h}}^{2}}\right)^{2} \\
& \times \frac{m_{\tilde{h}}^{3}\left(\cos \alpha v_{H}-\sin \alpha v_{S}\right)^{2}}{v_{H}^{2} v_{S}^{2}} .
\end{aligned}
$$

The singlet scalar $\tilde{s}$ can decay to pair of DM if kinematically allowed. This is the missing energy signal in the collider. The decay width is

$$
\Gamma(\tilde{s} \rightarrow \bar{\chi} \chi)=\frac{y_{\chi}^{2} \cos ^{2} \alpha}{8 \pi} m_{\tilde{s}}\left(1-\frac{4 m_{\chi}^{2}}{m_{\tilde{s}}^{2}}\right)^{3 / 2} .
$$

The SM Higgs $\tilde{h}$ can also decay to DM pair, with a similar decay width of $\tilde{s}$ by changing $\cos ^{2} \alpha$ to $\sin ^{2} \alpha$ and $m_{\tilde{s}}$ to $m_{\tilde{h}}$,

$$
\Gamma(\tilde{h} \rightarrow \bar{\chi} \chi)=\frac{y_{\chi}^{2} \sin ^{2} \alpha}{8 \pi} m_{\tilde{h}}\left(1-\frac{4 m_{\chi}^{2}}{m_{\tilde{h}}^{2}}\right)^{3 / 2} .
$$

In this model, the invisible decay branching ratios for $\tilde{s}$ and $\tilde{h}$ are

$$
\mathrm{BR}(\tilde{s} \rightarrow \mathrm{inv})=\frac{\Gamma(\tilde{s} \rightarrow \bar{\chi} \chi)}{\Gamma(\tilde{s} \rightarrow \bar{\chi} \chi)+\sin ^{2} \alpha \Gamma_{\tilde{h}, \text { tot }}^{\mathrm{SM}}\left(m_{\tilde{s}}\right)},
$$

$$
\operatorname{BR}(\tilde{h} \rightarrow \text { inv })=\frac{\Gamma(\tilde{h} \rightarrow \bar{\chi} \chi)+\Gamma(\tilde{h} \rightarrow \tilde{s} \tilde{s}) \mathrm{BR}^{2}(\tilde{s} \rightarrow \text { inv })}{\Gamma(\tilde{h} \rightarrow \bar{\chi} \chi)+\Gamma(\tilde{h} \rightarrow \tilde{s} \tilde{s})+\cos ^{2} \alpha \Gamma_{\tilde{h}, \text { tot }}^{\mathrm{SM}}}
$$

The mass of the singlet scalar relevant for the study of exotic $Z$ decays is $m_{\tilde{s}} \lesssim m_{Z}$. If $m_{\chi}<\frac{1}{2} m_{\tilde{s}}$, the singlet decays to DM, leading to missing energy signals.

\section{DM relic abundance, indirect and direct searches, and collider constraints}

Relic abundance and indirect detection.-In this model, the s-channel annihilation $\bar{\chi} \chi \rightarrow \bar{f} f$ is the dominant process for the thermal DM freeze-out. This process is p-wave suppressed, because the mediator is $C P$ even, while the initial state is $C P$ odd [85]. The analytic expression for the cross section can be written as 


$$
\begin{aligned}
\sigma v(\bar{\chi} \chi \rightarrow \bar{f} f)= & \frac{N_{C}}{8 \pi} \sin ^{2} \alpha \cos ^{2} \alpha y_{\chi}^{2} y_{f}^{2} \\
& \times \frac{\left(1-4 \frac{m_{f}^{2}}{s}\right)^{3 / 2}\left(s-4 m_{\chi}^{2}\right)\left(m_{\tilde{h}}^{2}-m_{\tilde{s}}^{2}\right)^{2}}{\left(\left(s-m_{\tilde{h}}^{2}\right)^{2}+m_{\tilde{h}}^{2} \Gamma_{\tilde{h}}^{2}\right)\left(\left(s-m_{\tilde{s}}^{2}\right)^{2}+m_{\tilde{s}}^{2} \Gamma_{\tilde{s}}^{2}\right)},
\end{aligned}
$$

where $y_{f} \equiv m_{f} / v$ and $s$ is the center-of-mass energy square. From this expression, it is clear that the annihilation cross section is p-wave from the term $s-4 m_{\chi}^{2} \propto v_{\text {rel }}^{2}$. As a result, DM indirect detection cannot put strong limits on this model, since the velocity dispersion of the galaxies is relatively slow. However, the temperature during DM freeze-out is relatively high, and $v_{\text {rel }} \simeq 1 / 3$. Therefore, the p-wave suppression is not dramatic during this period.

In the DM relic abundance calculation, we consider the fermions in the final states if the annihilations are kinetically allowed. In the mass range of $m_{b}<m_{\chi}<m_{Z} / 2$, the final states of quarks (b and c) and the $\tau$ lepton are included. The computation of the thermal relic density is restricted to $m_{\chi}>1.5 \mathrm{GeV}$. For the smaller DM mass, QCD nonperturbative effects and some hadronic channels should be considered. To avoid other limits, we choose $m_{\chi}$ close to $m_{\tilde{s}} / 2$ in Fig. 2. For the nonresonance case, relic abundance does not lead to competitive limits.

Direct detection.-The DM $\chi$ scattering with nuclei is mediated by t-channel scalar $\tilde{s}$ and $\tilde{h}$, which give the possibility to detect DM via spin-independent direct detection. The spin-independent scattering cross section with a nucleon is [86]

$$
\sigma_{S I}=\frac{\mu_{n}^{2} f_{n}^{2} m_{n}^{2}}{\pi v_{H}^{2}} g_{\chi}^{2} \sin ^{2} \alpha \cos ^{2} \alpha\left(\frac{1}{m_{\tilde{h}}^{2}-m_{\tilde{S}}^{2}}\right)^{2},
$$

where $\mu_{n}$ is the reduced mass between the DM and nucleon, $f_{n} \approx 0.3$ is the Higgs-nucleon coupling, and $m_{n}$ is the nucleon mass. We compare $\sigma_{S I}$ with the limits from XENON1T [69], LUX [70], PANDAX-II [71], and CRESST-II [72] as well as CDMSlite [73] for low mass $\mathrm{DM}$, and show the constraints in Fig. 2. The limits drop around $m_{\chi} \sim 10 \mathrm{GeV}$, because below this mass Xenon scintillators loose their sensitivity and CDMSlite becomes the dominant one.

Existing collider constraints.-The current LHC limits from the run I combination of ATLAS and CMS data constrain $\operatorname{BR}(h \rightarrow$ inv $) \leq 0.23$ at $95 \%$ C.L. $[87,88]$. Following the $\tilde{h}$ invisible decay branching ratio in Eq. (11), the limits on mixing angle $\sin \alpha$ are given in Fig. 2, labeled as " $\mathrm{BR}_{\mathrm{inv}}^{\tilde{h}}<0.23$." We also add the HL-LHC $\left(3 \mathrm{ab}^{-1}\right)$ and future $e^{+} e^{-}$collider projections on invisible Higgs search, which leads to $95 \%$ C.L. limits $\mathrm{BR}_{\text {inv }}^{\tilde{h}} \lesssim$ $0.08-0.16[89,90]$ and $\mathrm{BR}_{\text {inv }}^{\tilde{h}} \lesssim 0.003$ [4,91]. Moreover, the global fit to Higgs data at the LHC 7 and $8 \mathrm{TeV}$ runs can constrain the single scaling factor to Higgs interactions, and this gives $\sin \alpha<0.33$ [92], which is also added in Fig. 2, labeled as " $\tilde{h}$ current global fit (LHC)." The HL-LHC can extend the reach to $\sin \alpha<0.28(0.20)$ using $300 \mathrm{fb}^{-1}$ $\left(3 \mathrm{ab}^{-1}\right)$ luminosity [93].

At LEP-II, a low mass Higgs has been searched in the $e^{+} e^{-} \rightarrow Z \rightarrow Z^{*} h$ channel, where $Z$ decays visibly and $h$ decays invisibly, with an integrated luminosity of $\sim 114 \mathrm{pb}^{-1}$ [61]. The Higgs bremsstrahlung process $\mathrm{Zh}$ is also used at higher $\sqrt{s}$ to set a limit on heavier Higgs up to $114.4 \mathrm{GeV}$ [94-96]. The searches can put constraints on $\sin \alpha$ for the similar process $Z \tilde{s}$, which we give in Fig. 2 and are labeled as "LEP-Zs-inv." For the on-shell production of $Z \tilde{s}$ at FCC-ee, the sensitivity on $\sin \alpha$ has been estimated to be $\sim 0.03$ for $m_{\tilde{s}}<100 \mathrm{GeV}$ [97]. The precision measurement of the Higgs bremsstrahlung cross section $\sigma(Z h)$ can reach the accuracy of $\mathcal{O}(0.3 \%-0.7 \%)$ expected from $5-10 \mathrm{ab}^{-1}[1,4,98]$, which can probe the scalar mixing down to $0.055-0.084$ [97], labeled as " $\delta \sigma(Z h) . "$

\section{Prospects from exotic $Z$ decay}

Exotic Z-decay sensitivity.-For the sensitivity at a Giga (Tera) $Z$ factory, we study the process $Z \rightarrow \tilde{s} Z^{*} \rightarrow$ $(\bar{\chi} \chi)+\ell^{+} \ell^{-}$, with the Feynman diagram in Fig. 1, where $\tilde{s}$ decays to DM particles and off-shell $Z^{*}$ goes to lepton pairs. We set constraints on $\sin \alpha$ using this process and plot them in Fig. 2. The previous LEP experiment [61] has searched the similar channel with $Z^{*}$ decay to both hadronic and leptonic channels. The details of the simulations and cuts are given in Sec. IV. D, where the limit on the exotic decay BR has been calculated. After calculating the exotic decay BR, one can translate the constraints of decay BR to physical variable $\sin \alpha$. We have compared our analysis with the LEP and found good agreement. To be more specific, given that "LEP-Zs-inv" has also worked on the $Z$ pole with an integrated luminosity $114 \mathrm{pb}^{-1}$, we normalize our result to the same luminosity and find the constraint is similar to the LEP.

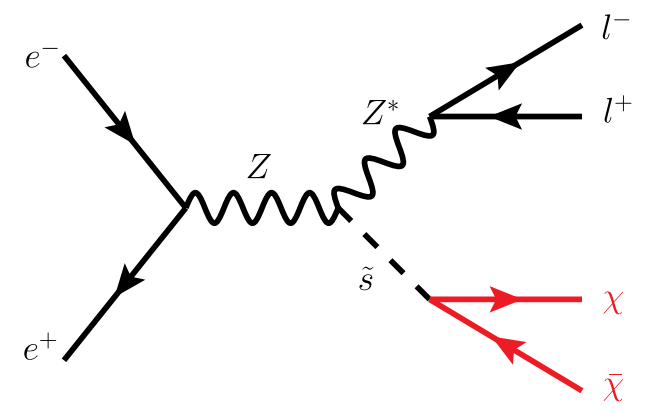

FIG. 1. The Feynman diagram for exotic $Z$ decay $Z \rightarrow \tilde{s} Z^{*} \rightarrow(\bar{\chi} \chi)+\ell^{+} \ell^{-}$. Note the $Z$ is produced on shell and followed by a three-body decay $\tilde{s} \ell^{+} \ell^{-}$, and the parentheses for $\bar{\chi} \chi$ indicate they are from the decay of a resonance. 

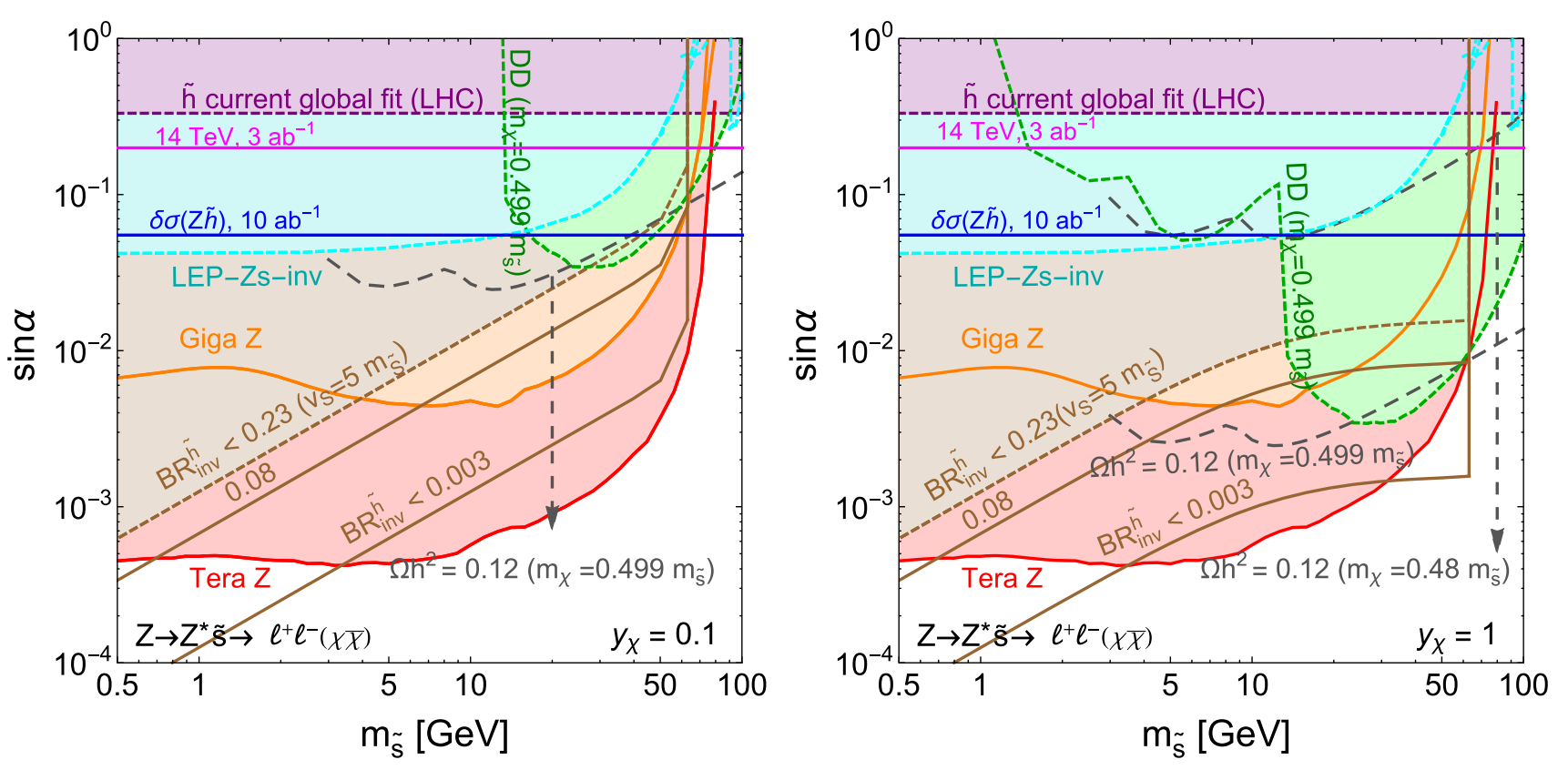

FIG. 2. The $95 \%$ C.L. sensitivity for $\sin \alpha$ from exotic $Z$ decay $Z \rightarrow \tilde{s} Z^{*} \rightarrow(\bar{\chi} \chi)+\ell^{+} \ell^{-}$at Giga (Tera) $Z$ factory, with $y_{\chi}=0.1(1)$ in the left (right) panels. We also compare with limits from DM direct detection, relic abundance, the invisible Higgs BR from the LHC [87,88] $\left(\mathrm{BR}_{\mathrm{inv}}^{\tilde{h}}<0.23\right)$, the high luminosity $\left(3 \mathrm{ab}^{-1}\right)$ LHC projection $\left(\mathrm{BR}_{\mathrm{inv}}^{\tilde{h}} \lesssim 0.08-0.16\right)$ [89,90] and future $e^{+} e^{-}$collider $\left(\mathrm{BR}_{\mathrm{inv}}^{\tilde{h}} \lesssim 0.003\right)$ [4,91], current and future Higgs global fits from ( $h$ current global fit) [92,93] with purple and magenta lines, low mass Higgs searches in invisible channels (LEP-Zs-inv) [61,94-96], and precision measurement of $\sigma(Z h)[\delta \sigma(Z h)][1,4,98]$. The dashed (solid) lines are for existing constraints (future prospects).

In the SM, Higgs can decay to a diphoton or $Z \gamma$ via a top loop and W loop. Due to the mixing between $\tilde{s}$ and $\tilde{h}$, the monophoton process $Z \rightarrow \gamma \tilde{s} \rightarrow \gamma(\bar{\chi} \chi)$ is possible. We have checked this process following the cuts in Sec. IV. B and found its constraint on $\sin \alpha$ is about 1 order of magnitude weaker than $Z \rightarrow \tilde{s} Z^{*} \rightarrow(\bar{\chi} \chi)+\ell^{+} \ell^{-}$. The main reason is monophoton decay is loop suppressed. Furthermore, the monophoton background is higher than the $\ell^{+} \ell^{-}+\not E$ background. Therefore, we do not put the constraint from the monophoton in Fig. 2.

Summary.-From Fig. 2, we see the relic abundance provides constraints on $\sin \alpha$ only in the fine-tuned scenario with $2 m_{\chi} \sim m_{\tilde{s}}$. The indirect detection does not provide limits because it is p-wave suppressed. The direct detection provides a useful constraint, which is not sensitive to the resonant mass of $m_{\tilde{s}} \sim 2 m_{\chi}$. At the same time, it depends on the size of the Yukawa coupling $y_{\chi}$. The existing and future Higgs global fit from the LHC does not provide competitive limits in comparison with precision measurement of $\sigma(Z h)$, while the invisible decay BR of SM Higgs provides a pretty good limit down to $\sin \alpha \sim \mathcal{O}\left(10^{-2}-10^{-3}\right)$ via the existing LHC data. At the HL-LHC $\left(3 \mathrm{ab}^{-1}\right)$, the reach of the invisible BR is about $0.08-0.16[89,90]$, which provides only a moderate improvement of the limit. The future sensitivity of $\mathrm{BR}_{\mathrm{inv}}^{h}$ is expected to reach $\sim 0.003$ at the future $e^{+} e^{-}$collider [1,4,91], which can improve the limits by a factor of $\sim 8.7$.

The proposed exotic $Z$ decay $Z \rightarrow \tilde{s} Z^{*} \rightarrow($ inv $)+\ell^{+} \ell^{-}$ can cover $\sin \alpha$ down to $\sim 10^{-2}\left(10^{-3}\right)$ for Giga $Z$ (Tera $Z$ ), and such constraints do not rely much on the values of $y_{\chi}$ and $\chi$ mass. The constraints from exotic $Z$ decay are superior than most of the existing and future searches, and only the invisible SM Higgs decay search at the future Higgs factories can provide competitive limits.

\section{B. Vector portal DM}

The vector portal, as another simple extension of the SM physics, employs a massive $U(1)$ dark photon connecting the SM sector and the hidden sector [17-22]. The searches for vector-portal DM and the vector field itself have attracted worldwide efforts (see the reviews in Refs. [6-8] and references therein). Various experiments, such as fixed target and $e^{+} e^{-}$and $p p$ colliders, are aiming to find such a dark photon, especially utilizing its coupling to $\ell^{+} \ell^{-}$. Aside from decaying to SM fermions, the invisible decays of the dark photon are directly related to DM, which can be searched by the radiative return process, meson decay, and missing energy events in scattering processes [6-8].

The dark photon $A^{\prime}$, as a $U(1)$ gauge field in the hidden sector, can mix with the SM hypercharge $U(1)_{Y}$ field $B_{\mu}$ through a renormalizable operator, 
$\mathcal{L}=-\frac{1}{4} B_{\mu \nu} B^{\mu \nu}-\frac{1}{4} A^{\prime}{ }_{\mu \nu} A^{\prime \mu \nu}+\frac{\epsilon}{2 c_{W}} B_{\mu \nu} A^{\prime \mu \nu}+\frac{1}{2} m_{A^{\prime}}^{2} A^{\prime \mu} A^{\prime}{ }_{\mu}$,

where $\epsilon$ is the kinetic mixing parameter and $c_{W}$ is the cosine of the weak angle. The mass of the dark photon, $m_{A^{\prime}}$, can be obtained from the Higgs mechanism in the dark sector. Interestingly, this underlying mechanism is related to our previous Higgs portal DM. We ignore here (possibly interesting) dynamics of the dark Higgs. ${ }^{2}$ We can always rotate away the kinetic mixing terms and work in the mass eigenstate basis. The rotation is nonunitary and is written down up to $\mathcal{O}\left(\epsilon^{2}\right)$ [99],

$$
\left(\begin{array}{c}
Z_{\mu} \\
A_{\mu} \\
A_{\mu}^{\prime}
\end{array}\right)=\left(\begin{array}{ccc}
1 & 0 & \frac{m_{A^{\prime}}^{2} t_{W}}{-m_{A^{\prime}}^{2} m_{Z}^{2}} \epsilon \\
0 & 1 & \epsilon \\
\frac{m_{Z}^{2} t_{W}}{m_{A^{\prime}}^{2}-m_{Z}^{2}} \epsilon & 0 & 1
\end{array}\right)\left(\begin{array}{c}
\tilde{Z}_{\mu} \\
\tilde{A}_{\mu} \\
\tilde{A}_{\mu}^{\prime}
\end{array}\right),
$$

where $t_{W}$ is the tangent of the weak angle. This formula does not apply to the region where the $A^{\prime}$ mass is pretty close to the mass of the $Z$ boson. In the rest of the paper, we work on the mass eigenstates of these gauge fields; without ambiguities, $\tilde{A}$ and $\tilde{A}^{\prime}$ are used to represent the mass eigenstates. After this rotation, the way that the currents couple to gauge fields is changed, and the interactions between vectors and currents up to $\mathcal{O}\left(\epsilon^{2}\right)$ are written as follows:

$$
\begin{aligned}
\mathcal{L}_{\text {int }}= & \tilde{Z}_{\mu}\left(g J_{Z}^{\mu}-g_{D} \frac{m_{Z}^{2} t_{W}}{m_{Z}^{2}-m_{A^{\prime}}^{2}} \epsilon J_{D}^{\mu}\right) \\
& +\tilde{A}_{\mu}^{\prime}\left(g_{D} J_{D}^{\mu}+g \frac{m_{A^{\prime}}^{2} t_{W}}{m_{Z}^{2}-m_{A^{\prime}}^{2}} \epsilon J_{Z}^{\mu}+e \epsilon J_{\mathrm{em}}^{\mu}\right) \\
& +\tilde{A}_{\mu} e J_{\mathrm{em} .}^{\mu} .
\end{aligned}
$$

The massless photon $\tilde{A}$ couples to the electromagnetic current $J_{\mathrm{em}}$. The dark photon couples to dark $U(1)$ currents $J_{D}$; after the field rotates, a $\epsilon$ suppressed coupling to $J_{\mathrm{em}}$ and $J_{Z}$ arises. The $\tilde{Z}$ boson couples to $J_{Z}$ and has the coupling to the dark currents with $\epsilon$ suppression.

\section{Scalar vector-portal DM}

Model.-In this model, we introduce a complex scalar as DM, charged under the $U(1)_{D}$, and this scalar DM interacts with the SM particles via the dark photon $A^{\prime}$. The relevant interactions can be written as follows:

\footnotetext{
${ }^{2}$ The mass of $A^{\prime}$ usually needs the Higgs mechanism to break $U(1)_{D}$ and obtain a vev; therefore, it requires a complex scalar $\phi$ charged under $U(1)_{D}$. It naturally provides an exotic $Z$-decay signature $Z \rightarrow A^{\prime} \phi$ from $Z-A^{\prime}$ mixing.
}

$\mathcal{L}_{S}=\left(\partial_{\mu} S+i g_{D} A^{\prime}{ }_{\mu} S\right)^{*}\left(\partial^{\mu} S+i g_{D} A^{\prime \mu} S\right)-m_{S}^{2} S^{*} S$.

For $m_{S}^{2}>0$ and considering $Z_{2}$ symmetry, $\langle S\rangle=0$, and $S$ is stable. From Eqs. (15) and (16), it is clear that there is coupling between $Z, A^{\prime}$, and $S$,

$$
\mathcal{L}_{S} \supset g_{D}^{2} S^{*} S\left(\tilde{A}^{\prime}{ }_{\mu}+\epsilon \frac{m_{Z}^{2} t_{W}}{\left(m_{A^{\prime}}^{2}-m_{Z}^{2}\right)} \tilde{Z}_{\mu}\right)^{2}
$$

which can provide an interesting signal for the exotic $Z$ decay, $\tilde{Z} \rightarrow \tilde{A}^{\prime} S^{*} S$, from the leading $\epsilon$ terms in the Lagrangian. To have this signal, we must have this process kinematically allowed, $m_{\tilde{A}^{\prime}}+2 m_{S}<m_{Z}$. We will focus on the region where $m_{S}>\frac{1}{2} m_{\tilde{A}^{\prime}}$, such that the $\tilde{A}^{\prime}$ decay dominantly to SM particles, rather than invisible DM pair. ${ }^{3}$

The spontaneous symmetry breaking through dark Higgs $\phi$ is a simple mechanism to give mass to $S$ and $A^{\prime}$. The difference from $S$ is that there is no exact $Z_{2}$ symmetry to make $\phi$ stable, but the Lagrangian is similar to Eq. (17),

$$
\begin{aligned}
\mathcal{L}_{\phi}= & \left(\partial_{\mu} \phi+i g_{D} A^{\prime}{ }_{\mu} \phi\right)^{*}\left(\partial^{\mu} \phi+i g_{D} A^{\prime \mu} \phi\right) \\
& +\lambda_{2} S^{*} S \Phi^{*} \Phi-\mu_{\phi}^{2}|\phi|^{2}-\frac{\lambda_{4}}{4}|\phi|^{4} .
\end{aligned}
$$

After symmetry breaking $\langle\phi\rangle \neq 0, A^{\prime}$ and $S$ get their mass. When $\phi$ is much heavier than $S$ and $A^{\prime}$, it can be integrated out, and Eq. (17) is enough to describe the process related to DM and various searches. When the $\phi$ mass is smaller than or comparable to the mass of $S$ and $A^{\prime}, \phi$ need to be considered. In this case, $\phi$ can be produced at a collider and decay back to $2 S$ or $2 \tilde{A}^{\prime}$.

$D M$ relic abundance and indirect detection.-If $m_{S}>m_{\tilde{A}^{\prime}}$, the dominant process controlling the freeze-out is $S S^{*} \rightarrow \tilde{A}^{\prime} \tilde{A}^{\prime}$. The thermal cross section is

$\sigma v\left(S S^{*} \rightarrow \tilde{A}^{\prime} \tilde{A}^{\prime}\right)=\frac{g_{D}^{4}}{16 \pi m_{S}^{2}} \frac{\left(8-8 y^{2}+3 y^{4}\right) \sqrt{1-y^{2}}}{\left(2-y^{2}\right)^{2}}$,

where $y \equiv m_{\tilde{A}^{\prime}} / m_{S}$. By taking $s \rightarrow 4 m_{S}^{2}$, the leading term tells us that this process is s-wave. This thermal cross section is not related to $\epsilon$, since the $\tilde{A}^{\prime}$ are produced on shell. On the other hand, in the regime where $m_{S}<m_{\tilde{A}^{\prime}}$, the dominant process is $S^{*} S \rightarrow \tilde{A}^{\prime} / Z \rightarrow \bar{f} f$ via the off-shell Z and $\tilde{A}^{\prime}$, and the thermal cross sections for $\sigma v\left(S S^{*} \rightarrow f \bar{f}\right)$ are given in Sec. VI. Since the thermal cross section is proportional to $\epsilon^{2}$, the relic abundance will rely on the size of the kinetic mixing. This can set the target for the search of exotic $Z$ decay. Without loss of generality, we will restrict to $m_{S}=0.8 m_{\tilde{A}^{\prime}}$ in the parameter space, to compare

${ }^{3}$ This assumption can be relaxed, and the constraints should be rescaled according to the branching ratio of $\tilde{A}^{\prime}$ to $\mathrm{SM}$ particles. 

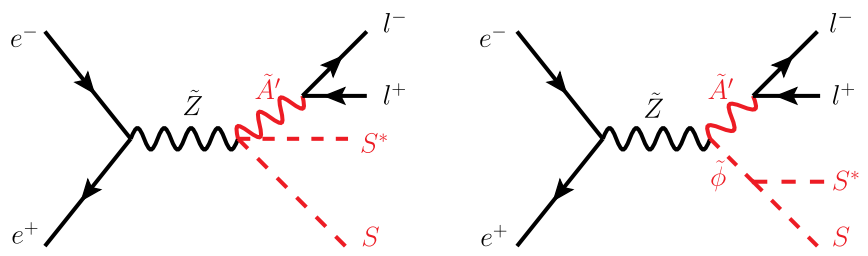

FIG. 3. The Feynman diagrams for the three-body decay process $\tilde{Z} \rightarrow \tilde{A}^{\prime} S S^{*} \rightarrow\left(\ell^{-} \ell^{+}\right) E$ from the vector-portal model with scalar DM and the Higgs bremsstrahlung process $\tilde{Z} \rightarrow$ $\tilde{A}^{\prime} \tilde{\phi} \rightarrow\left(\ell^{-} \ell^{+}\right)(\mathscr{E})$.

various limits from the complementary experiments, shown in Fig. 4.

$S^{*} S \rightarrow \tilde{A}^{\prime} / Z \rightarrow \bar{f} f$ is $\mathrm{p}$-wave suppressed, which can be understood from the $C P$ symmetry of the initial state [85]. As we discussed before, the p-wave annihilation has the suppressed signal of the indirect detection. Therefore, the corresponding limit is negligible.

Direct detection.-The scattering of $S$ off nuclei is mediated by t-channel $\tilde{A}^{\prime}$ and $\tilde{Z}$. Interestingly, the contribution from $\tilde{Z}$ exchange has been canceled by the one from $\tilde{A}^{\prime}$ coupling to the $J_{Z}$ current [97]; hence, only $\tilde{A}^{\prime}$ coupling to the $J_{\mathrm{em}}$ current should be considered, which can be seen directly from Eq. (16). Therefore, the spin-independent scattering cross section for $S$ and the nucleon has a simple expression and is given below,

$$
\sigma_{n}^{\mathrm{SI}} \simeq \frac{e^{2} g_{D}^{2} \epsilon^{2} \mu_{S n}^{2}}{2 \pi m_{\tilde{A}^{\prime}}^{4}}
$$

where $\mu_{S n}=m_{S} m_{n} /\left(m_{S}+m_{n}\right)$ is the reduced mass of dark matter $S$ and nucleon $n$ and $e$ is the electron charge. We add

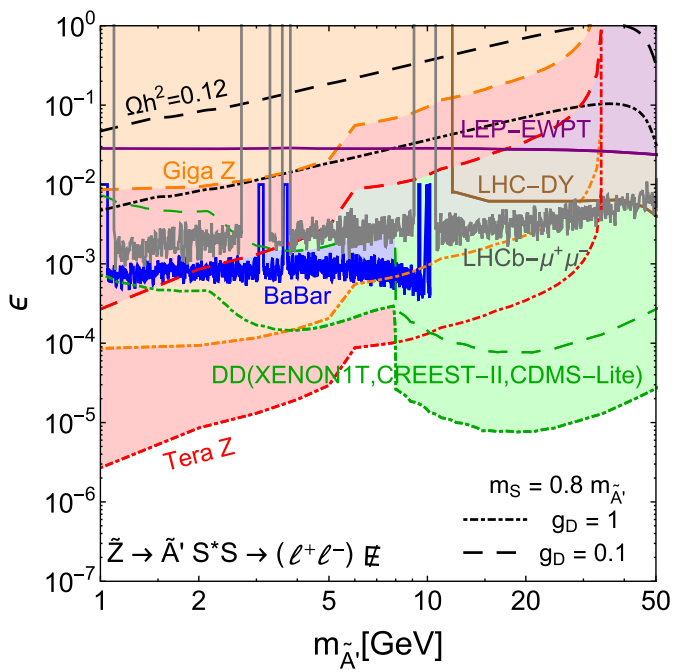

the direct detection constraints as the green shaded area in Fig. 4.

Existing collider limits.-Focusing on the region of $m_{\tilde{A}^{\prime}}<2 m_{S}$, the decay mode of the dark photon, $\tilde{A}^{\prime} \rightarrow \ell^{+} \ell^{-}$, is the key channel to look for in the experiments: beam-dump, fixed target, collider, and rare meson decay. In Fig. 4, we present the constraints from the experiments having the leading limits currently. There are also limits from the LEP via electroweak precision observables [41]. For constraints from the LHC, the inclusive Drell-Yan process $p p \rightarrow \tilde{A}^{\prime} \rightarrow \ell^{+} \ell^{-}$can be used to constrain $\epsilon$ with the LHC $8 \mathrm{TeV}$ data $[100,101]$, which provides a stronger bound than the electroweak precision bounds $[42,102,103]$. For low mass $m_{\tilde{A}^{\prime}} \sim \mathcal{O}(\mathrm{GeV})$, the limits from the $B$ factory are the leading ones from measuring visible decay products of the dark photon, such as BABAR 2014 [104] having the limits of $\epsilon \lesssim 10^{-3}$. Recently, the LHCb [105] performed a dark photon search using the inclusive dimuon data. This will give the leading constraints in the mass window of $(10 \mathrm{GeV}, 50 \mathrm{GeV})$.

Exotic Z-decay search.-The first process we consider is the three-body decay $\tilde{Z} \rightarrow \tilde{A}^{\prime} S^{*} S \rightarrow\left(\ell^{+} \ell^{-}\right) \mathscr{E}$ shown in the left panel of Fig. 3. The limit on the exotic Z-decay branching ratio is given in Sec. IV. D. Here, we take the mass range of $\tilde{A}^{\prime}, m_{S}<m_{\tilde{A}^{\prime}}<2 m_{S}$, such that $\tilde{A}^{\prime}$ will not dominantly decay to invisible DMs, and DM relic density depends on the kinetic mixing $\epsilon$. To constrain kinetic mixing coupling $\epsilon$, we fix the other relevant parameters, $g_{D}=0.1(1)$ and the mass ratio $m_{S} / m_{\tilde{A}^{\prime}}=0.8$. The corresponding limit for $\epsilon$ as a function of $m_{\tilde{A}^{\prime}}$ is given in Fig. 4. The range of $m_{\tilde{A}^{\prime}}$ starts from $1 \mathrm{GeV}$. For smaller masses, other constraints like beam dump experiments become

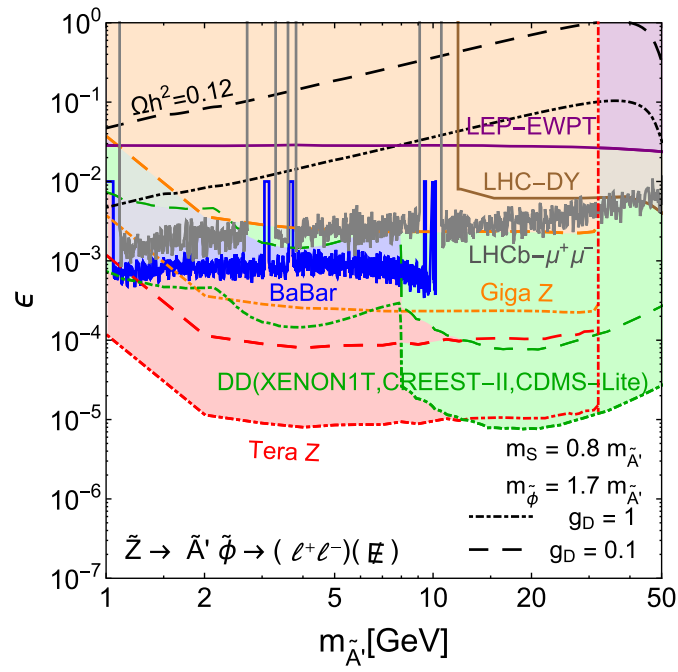

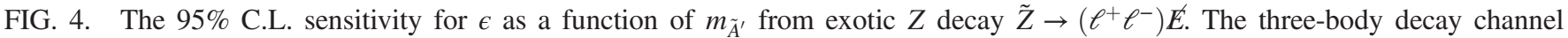
$\tilde{Z} \rightarrow \tilde{A}^{\prime} S^{*} S \rightarrow\left(\ell^{+} \ell^{-}\right) E^{\prime}$ is shown in the left panel, while the two-body cascade decay channel $\tilde{Z} \rightarrow \tilde{A}^{\prime} \tilde{\phi} \rightarrow\left(\ell^{+} \ell^{-}\right)\left(\mathscr{E}^{6}\right)$ is shown in the right panel. We take $g_{D}=0.1$ and $1, m_{S}=0.8 m_{\tilde{K}}$. The constraints from exotic $Z$ decay are labeled as Giga (Tera) $Z$, and also we show an illustrative line for LEP luminosity $114 \mathrm{pb}^{-1}$. We also show limits from relic abundance, direct detection, and existing collider searches for comparison. 
quite strong. Moreover, the exotic $Z$ search begins to lose its efficiency due to the small separation of the lepton pair from $\tilde{A}^{\prime}$ decay.

In addition to the three-body decay topology, we can also have the two-body cascade decay $\tilde{Z} \rightarrow \tilde{A}^{\prime} \tilde{\phi} \rightarrow$ $\left(\ell^{+} \ell^{-}\right)\left(S^{*} S\right)$, shown in the right panel of Fig. 3. This channel has resonance in both the lepton pair invariant mass and invisible mass. We still consider the regime $m_{S}<m_{\tilde{A}^{\prime}}<2 m_{S}$. Therefore, $\tilde{A}^{\prime}$ decays into lepton pairs. We assume $m_{\tilde{\phi}}<2 m_{\tilde{A}^{\prime}}$ and very small Higgs portal mixing, so that $\tilde{\phi}$ decay to SM particles via Higgs mixing can be neglected. Therefore, the decay branching ratio of $\tilde{\phi} \rightarrow S^{*} S$ is $\sim 100 \%$. In the right panel of Fig. 4, we constrain $\epsilon$ as a function of $m_{\tilde{A}^{\prime}}$, with $m_{S}=0.8 m_{\tilde{A}^{\prime}}$ and $m_{\tilde{\phi}}=1.7 m_{\tilde{A}^{\prime}}$. Given that $\tilde{\phi}$ has negligible coupling to the SM sector, the relic abundance, indirect detection, and direct detection are similar to the left panel of Fig. 4.

Summary.-As shown in Fig. 4, the LEP electroweak precision test, LHC Drell-Yan, BABAR radiative return, and $\mathrm{LHCb}$ dimuon inclusive searches can provide the direct constraints on $\epsilon$. For $m_{\tilde{A}^{\prime}}<10 \mathrm{GeV}, B A B A R$ bounds $\epsilon \lesssim 10^{-3}$, while LHC Drell-Yan and LHCb provide complementary limits $\epsilon \gtrsim 5 \times 10^{-3}$ for $m_{\tilde{A}^{\prime}}>10 \mathrm{GeV}$. The LEP electroweak precision test is the weakest constraint among the three.

The hint from the DM relic abundance and the constraints from direct detection and exotic $Z$ decay rely on coupling $g_{D}$. For a fixed $m_{\tilde{A}^{\prime}}$, the DM annihilation cross section and direct detection scattering cross section are proportional to $g_{D}^{2}$. The coupling for the four-point vertex $\tilde{Z}_{\mu} \tilde{A}^{\prime \mu} S^{*} S$ is proportional to $\epsilon g_{D}^{2}$, while the coupling for the three-point vertex $\tilde{Z}_{\mu} \tilde{A}^{\prime \mu} \tilde{\phi}$ is proportional to $\epsilon g_{D} m_{\tilde{A}^{\prime}}$. Therefore, the three-body decay width is proportional to $g_{D}^{4}$, while the two-body cascade decay width is proportional to $g_{D}^{2}$. For $g_{D}=1$, we see Tera $Z$ could provide the strongest bounds at low $m_{\tilde{A}^{\prime}}$, while direct detection provides comparable limits to exotic $Z$ decay at high $m_{\tilde{A}^{\prime}}$.

In comparison with the three-body cascade decay, one might expect a better constraint from two-body cascade decay because there are resonances in both the lepton pair and missing energy in this topology, while three-body decay only has one resonance in the lepton pair. This intuition is indeed correct as the sensitivity on the exotic decay BR is better for two-body cascade decay than three-body decay in Sec. IV. D, but the difference is not significant. The limits on $\epsilon$ in Fig. 4 involve more parameters and couplings, which modify the dependence of $m_{\tilde{A}^{\prime}}$.

For $m_{\tilde{A}^{\prime}} \sim 1 \mathrm{GeV}$, three-body decay loses less efficiency from the lepton separation requirement than two-body cascade decay, since the energy of $\tilde{A}^{\prime}$ in three-body decay is generally softer than in two-body cascade decay.

In summary, the exotic $Z$-decay search in both topologies can provide good reach in $\epsilon$, which is complementary to and competitive with other constraints.

\section{2. (Inelastic) vector-portal fermionic DM}

For vector-portal fermionic DM, we consider the inelastic DM model here. The constrains and future collider search of $Z$ decays are similar to magnetic inelastic dark matter, which will be explored in Sec. III. C.

Starting from the fermionic DM charged under the dark sector $U(1)_{D}$, we can write down its Dirac mass term $m_{D} \bar{\chi} \chi$, and its Majorana mass is obtained through Yukawa interaction with a scalar $\Phi$. The Lagrangian is

$$
\begin{aligned}
\mathcal{L}_{F}= & \bar{\chi} i \not \partial \chi+g_{D} \bar{\chi} A_{\mu}^{\prime} \gamma^{\mu} \chi-m_{D} \bar{\chi} \chi \\
& +\left(\Phi^{*}\left(y_{L} \bar{\chi}^{c} P_{L} \chi+y_{R} \bar{\chi}^{c} P_{R} \chi\right)+\text { H.c. }\right) .
\end{aligned}
$$

The ratio of $U(1)_{D}$ charge of $\Phi$ and $\chi$ equals 2. Once $\Phi$ gets a vev, the DM $\chi$ gets Majorana mass along with its Dirac mass.

As a result, the Dirac fermion splits itself into two Majorana fermions, which provide the DM $\chi_{1}$ and its excited state $\chi_{2}$, dubbed "inelastic dark matter" (IDM) $[106,107]$.

We work with the Weyl spinor and analyze the interactions for $\chi_{1}$ and $\chi_{2}$. If we write $\chi=\left\{\eta, \xi^{\dagger}\right\}$, the mass term is given as $[106,108]$

$$
-\mathcal{L}_{F} \supset \frac{1}{2}\left(\begin{array}{ll}
\eta & \xi
\end{array}\right)\left(\begin{array}{cc}
m_{\eta} & m_{D} \\
m_{D} & m_{\xi}
\end{array}\right)\left(\begin{array}{l}
\eta \\
\xi
\end{array}\right)+\text { H.c. },
$$

where $m_{\eta}=-\sqrt{2} y_{L} v_{D}$ and $m_{\xi}=-\sqrt{2} y_{R}^{*} v_{D}$. The mass matrix can be diagonalized by a rotation,

$$
\left(\begin{array}{l}
\eta \\
\xi
\end{array}\right)=\left(\begin{array}{cc}
\cos \beta & \sin \beta \\
-\sin \beta & \cos \beta
\end{array}\right)\left(\begin{array}{l}
\chi_{1} \\
\chi_{2}
\end{array}\right)
$$

where $\tan 2 \beta=2 m_{D} /\left(m_{\xi}-m_{\eta}\right)$. The masses of $\chi_{1}$ and $\chi_{2}$ are

$$
m_{\chi_{1}, \chi_{2}}=\frac{1}{2}\left(m_{\eta}+m_{\xi} \mp \sqrt{\left(m_{\eta}-m_{\xi}\right)^{2}+4 m_{D}^{2}}\right) .
$$

The vector current of the DM couples to $U(1)_{D}$ gauge field $A^{\prime}$. We can write both of them in the mass basis as follows,

$$
\begin{aligned}
\mathcal{L}_{F} \supset & A^{\prime \mu} J_{\mu}=\left(\tilde{A}^{\prime \mu}+t_{W} \epsilon \frac{m_{Z}^{2}}{\left(m_{A^{\prime}}^{2}-m_{Z}^{2}\right)} \tilde{Z}^{\mu}\right)\left(\eta^{\dagger} \bar{\sigma}^{\mu} \eta-\xi^{\dagger} \bar{\sigma}^{\mu} \xi\right) \\
= & \left(\tilde{A}^{\prime \mu}+t_{W} \epsilon \frac{m_{Z}^{2}}{\left(m_{A^{\prime}}^{2}-m_{Z}^{2}\right)} \tilde{Z}^{\mu}\right) \\
& \times\left(\frac{1}{x}\left(\chi_{1}^{\dagger} \bar{\sigma}^{\mu} \chi_{1}-\chi_{2}^{\dagger} \bar{\sigma}^{\mu} \chi_{2}\right)\right. \\
& \left.-\frac{2 m_{D}}{\left(m_{\xi}-m_{\eta}\right) x}\left(\chi_{1}^{\dagger} \bar{\sigma}^{\mu} \chi_{2}+\chi_{1}^{\dagger} \bar{\sigma}^{\mu} \chi_{2}\right)\right),
\end{aligned}
$$

where we have defined $x \equiv \sqrt{1+4 m_{D}^{2} /\left(m_{\xi}-m_{\eta}\right)^{2}}$. The scalar interaction with DM can be written as 


$$
\begin{aligned}
\mathcal{L}_{F} & \supset \frac{-1}{2}\left(1+\frac{\phi}{v_{D}}\right)\left(m_{\eta} \eta \eta+m_{\xi} \xi \xi\right)+\text { H.c. } \\
= & \frac{-1}{2}\left(1+\frac{\cos \alpha \tilde{\phi}-\sin \alpha \tilde{h}}{v_{D}}\right) \\
& \times\left(\frac{1}{2}\left(m_{\xi}+m_{\eta}+\frac{-m_{\xi}+m_{\eta}}{x}\right) \chi_{1} \chi_{1}\right. \\
& \left.+\frac{1}{2}\left(m_{\xi}+m_{\eta}+\frac{m_{\xi}-m_{\eta}}{x}\right) \chi_{2} \chi_{2}-\frac{2 m_{D}}{x} \chi_{1} \chi_{2}\right) .
\end{aligned}
$$

There are two interesting parameter regions for this model. In the first one, the Majorana mass is much larger than its Dirac mass, $m_{\eta}, m_{\xi} \gg m_{D}$, such that the mixing angle $\beta$ is small and the masses of $\chi_{1}$ and $\chi_{2}$ have small corrections to its Majorana masses, where $m_{\chi_{1}} \approx$ $m_{\eta}+m_{D}^{2} /\left(m_{\eta}-m_{\xi}\right)$ and $m_{\chi_{2}} \approx m_{\xi}+m_{D}^{2} /\left(m_{\xi}-m_{\eta}\right)$. The interactions with the vector boson and scalar are mainly diagonal, while the off-diagonal interactions for $\chi_{1}$ and $\chi_{2}$ are suppressed.

In the second case, the Dirac mass is dominant, $m_{\eta}$, $m_{\xi} \ll m_{D}$. Therefore, the mixing angle $\beta$ is very close to its maximal value $\pi / 4$. The mass of $\chi_{1}$ and $\chi_{2}$ are $m_{\chi_{1}} \approx$ $m_{D}-\left(m_{\xi}+m_{\eta}\right) / 2$ and $m_{\chi_{2}} \approx m_{D}+\left(m_{\xi}+m_{\eta}\right) / 2$, with the mass splitting $\Delta=m_{\xi}+m_{\eta}$. We have $x \approx 2 m_{D} /\left(m_{\xi}-m_{\eta}\right)$ and $|x| \gg 1$. This suggests that the diagonal interactions with the vector boson are suppressed while the off-diagonal interaction to $\chi_{1}$ and $\chi_{2}$ is dominant. For the special case of $m_{\xi}=m_{\eta}$, the diagonal interaction with the vector boson vanishes. Since the IDM relies on the off-diagonal interactions with the vector boson, the DM scattering only happens when the final states are its excited ones and provides very different phenomenology from ordinary elastic scattering in direct detection [106]. However, for scalar interactions, the diagonal interaction with the fermionic DM is proportional to $m_{\xi}+m_{\eta}$, while the offdiagonal interaction is proportional to $m_{\xi}-m_{\eta}$. The scalar mediation to diagonal terms can potentially spoil the IDM setup when the Higgs portal coupling is large.

Coming back to the exotic $Z$ decays, we see that vectorportal IDM motivates the exotic decays of $\tilde{Z} \rightarrow \chi_{2} \chi_{1}$ and $\chi_{2} \chi_{2}$, followed by the subsequent cascade decay $\chi_{2} \rightarrow$ $\tilde{A}^{\prime} \chi_{1}, \tilde{\phi} \chi_{1}$ and $\tilde{A}^{\prime}, \tilde{\phi} \rightarrow \bar{f} f, \chi_{1} \chi_{1}$. This shows that IDM with the vector portal can motivate the topologies of exotic $Z$ decay in Sec. IV.

\section{Magnetic inelastic DM and Rayleigh DM}

The coupling of DM to the Standard Model particles can be very weak. One possible scenario is that the hidden sector interacts with the Standard Model via highdimensional operators. The representative models, the magnetic inelastic DM (MIDM) and Rayleigh DM model (RayDM) [36-40], are introduced, and their relevance to the exotic $Z$ decay is studied in this section.

\section{Model}

The two models, the MIDM and RayDM, can be derived from the same UV model [40],

$$
\begin{aligned}
\mathcal{L}= & \bar{\chi}\left(i \not \supset-m_{\chi}\right) \chi-\frac{1}{2} \delta m \bar{\chi}^{c} \chi+\bar{\psi}\left(i \not D-M_{\psi}\right) \psi \\
& +\left(D^{\mu} \phi\right)^{\dagger}\left(D_{\mu} \phi\right)-M_{\phi}^{2} \phi^{\dagger} \phi+(\lambda \bar{\psi} \chi \phi+\text { H.c. }) .
\end{aligned}
$$

$\chi$ is fermionic DM with a Dirac mass term $m_{\chi}$ and Majorana mass term $\delta m$. It interacts with scalar $\phi$ and another fermion $\psi$ via a Yukawa coupling. The Dirac and Majorana mass terms can split DM $\chi$ into two Majorana fermions $\chi_{1}$ and $\chi_{2}$, where we assume $m_{\chi_{2}}>m_{\chi_{1}}$. The fermion $\psi$ and scalar $\phi$ have the same charge under SM gauge group $\mathrm{SU}(2)_{L} \times \mathrm{U}(1)_{Y}$ [40]. The dark matter will couple to photon via a $\psi$ and $\phi$ loop. Integrating out $\psi$ and $\phi$ will generate two higher-dimensional operators. The first operator is the MIDM operator [36-38], and the second is the RayDM operator [39]. Both of them are given below:

$$
\begin{aligned}
O_{\mathrm{MIDM}} & =\frac{1}{\Lambda_{\mathrm{MIDM}}} \bar{\chi}_{2} \sigma^{\mu \nu} \chi_{1} B_{\mu \nu}+\text { H.c. }, \\
O_{\mathrm{RayDM}} & =\frac{1}{\Lambda_{\mathrm{RayDM}}^{3}} \bar{\chi}_{1} \chi_{1} B^{\mu \nu} B_{\mu \nu} .
\end{aligned}
$$

Note there are also operators including $\gamma_{5}$ in the DM bilinear, which corresponds to the electric dipole operator. For RayDM, the corresponding one is

$$
O_{\mathrm{RayDM}}^{\gamma_{5}}=\frac{i}{\Lambda_{\mathrm{RayDM}}^{3}} \bar{\chi}_{1} \gamma_{5} \chi_{1} B^{\mu \nu} \tilde{B}_{\mu \nu}
$$

where $\tilde{B}_{\mu \nu}=\epsilon_{\mu \nu \alpha \beta} B^{\alpha \beta}$ and $\epsilon_{\mu \nu \alpha \beta}$ is the antisymmetric LeviCività symbol. The interaction scale $\Lambda$ has been calculated in Ref. [40],

$$
\frac{1}{\Lambda_{\mathrm{MIDM}}} \approx \frac{\lambda^{2} g_{Y}}{64 \pi^{2} M_{\psi}}, \quad \frac{1}{\Lambda_{\mathrm{RayDM}}^{3}} \approx \frac{\lambda^{2} g_{Y}^{2}}{48 \pi^{2} M_{\psi}^{3}},
$$

where we have assumed that $\psi$ and $\phi$ are singlet under $S U(2)_{L}$ and are charged under $U(1)_{Y}$. In Eq. (31), we have assumed the $\phi$ mass is similar to $M_{\psi}$, and we take the form factor function to be $\mathcal{O}(1)$. These two operators can lead to the cascade decay $Z \rightarrow \chi_{2} \chi_{1} \rightarrow\left(\chi_{1} \gamma\right) \chi_{1}$ and the three-body decay $Z \rightarrow \chi_{1} \chi_{1} \gamma$ at the $Z$ factory, with Feynman diagrams given in Fig. 5. In the exotic $Z$-decay study, we will choose a significant mass splitting between $\chi_{1}$ and $\chi_{2}$ to get a hard photon signal which can be detected at $Z$ factories.

With this setup, we see that decay topologies $Z \rightarrow$ $\chi_{2} \chi_{1} \rightarrow\left(\chi_{1} \gamma\right) \chi_{1}$ and $Z \rightarrow \chi_{1} \chi_{1} \gamma$ in Fig. 5 can be easily achieved. In the perspective of model building, the cascade decay channel $Z \rightarrow \chi_{2} \chi_{2} \rightarrow\left(\chi_{1} \gamma\right)\left(\chi_{1} \gamma\right)$ would be more complicated. In particular, if $\chi_{2}$ is Majorana fermion, the dipole term $\bar{\chi}_{2} \sigma_{\mu \nu} \chi_{2}$ will vanish. If one would add new 

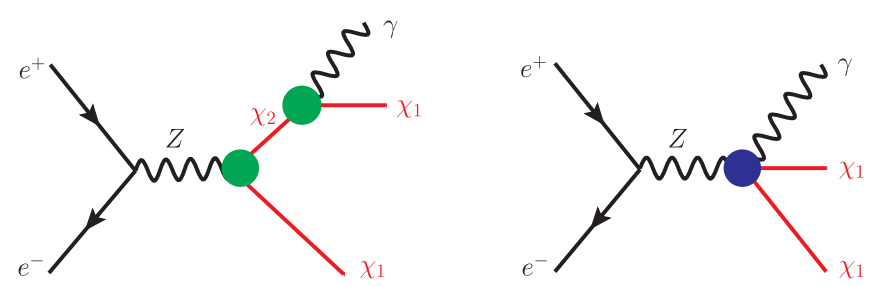

FIG. 5. The Feynman diagrams for the cascade decay process $Z \rightarrow \chi_{2} \chi_{1} \rightarrow \chi_{1} \chi_{1} \gamma$ from $O_{\mathrm{MIDM}}$ and the three-body process $Z \rightarrow \chi_{1} \chi_{1} \gamma$ from $O_{\text {RayDM }}$.

species of Dirac fermion DM $\chi$, then the Yukawa term in Eq. (28) would become $\lambda_{i} \bar{\psi} \chi_{i} \phi$, where $i$ is the number of species [109]. In this case, one can have $\bar{\chi}_{i} \sigma_{\mu \nu} \chi_{j}$ in the MIDM operator and $\bar{\chi}_{i} \chi_{j}$ in the RayDM operator, which provide rich cascade decays for exotic $Z$ decay.

\section{DM relic abundance, indirect and direct searches, and collider constraints}

Relic abundance and indirect detection.-We focus on the case in which there is a significant mass splitting between $\chi_{2}$ and $\chi_{1}$, which can give rise to an interesting photon signal in exotic $Z$ decay. In this case, the relevant annihilation initial state contains only $\chi_{1}$. The annihilation rate is dominated by the Rayleigh operator into $\gamma \gamma, \gamma Z, Z Z$, and $W^{+} W^{-}$. For the mass range $m_{\chi_{1}}<m_{Z}$, we find only the following annihilation cross section relevant [39],

$\sigma v\left(\chi_{1} \chi_{1} \rightarrow \gamma \gamma\right)_{\mathrm{MIDM}}=\frac{\cos ^{2} \theta_{w} m_{\chi_{1}}^{2}}{\pi \Lambda_{\mathrm{MIDM}}^{4}} \frac{16 y^{6}-9 y^{4}-2 y^{2}-2}{y^{4}\left(y^{2}+2\right)^{2}}$,

$$
\begin{aligned}
& \sigma v\left(\chi_{1} \chi_{1} \rightarrow \gamma \gamma\right)_{\text {RayDM }}=\frac{\cos ^{2} \theta_{w}}{\pi} \frac{m_{\chi_{1}}^{4}}{\Lambda_{\mathrm{RayDM}}^{6}} v_{\mathrm{rel}}^{2}, \\
& \sigma v\left(\chi_{1} \chi_{1} \rightarrow \gamma \gamma\right)_{\mathrm{RayDM}}^{\gamma_{5}}=\frac{16 \cos ^{2} \theta_{w}}{\pi} \frac{m_{\chi_{1}}^{4}}{\Lambda_{\mathrm{RayDM}}^{6}},
\end{aligned}
$$

where $y \equiv m_{\chi_{2}} / m_{\chi_{1}}$. The two annihilation cross sections for RayDM are for $O_{\text {RayDM }}$ and $O_{\text {RayDM }}^{\gamma_{5}}$, respectively, where the former one is p-wave while the second one is s-wave. The annihilation process for the MIDM scenario is two-loop suppressed. This can be seen in Eqs. (32), (33), and (34) through the dependence on $\Lambda_{\text {MIDM }}$ and $\Lambda_{\text {RayDM }}$, respectively. The annihilation into gamma-ray lines is constrained by the Fermi-LAT search [74] (blue shaded region) and also by the CMB [77] (purple shaded region), which we use to constrain $M_{\psi}$ as a function of $m_{\chi}$ in Fig. 6. The long dashed lines are for $O_{\text {RayDM }}^{\gamma_{5}}$, while the dashed lines are for $O_{\text {RayDM }}$, which is very weak due to the p-wave suppression.

Direct detection.-In the case of large splitting, only Majorana $\chi_{1}$ is relevant for direct detection because inelastic scattering into $\chi_{2}$ is kinetically forbidden. Therefore, the scattering cross section is dominated by the loop exchange of two photons from the Rayleigh operator, and the spin-independent cross section per nucleon is given below [39],

$$
\sigma_{n}^{\mathrm{SI}} \approx \frac{4 \alpha_{\mathrm{EM}}^{2} Z^{4}}{\pi^{2} A^{4}} \frac{m_{N}^{2} Q_{0}^{2}}{\Lambda_{\mathrm{RayDM}}^{6}},
$$

where $m_{N}$ is the mass of nuclei $N, A$ is the nucleon number, $Z$ is the proton number of nuclei, and $Q_{0}$ is the nuclear
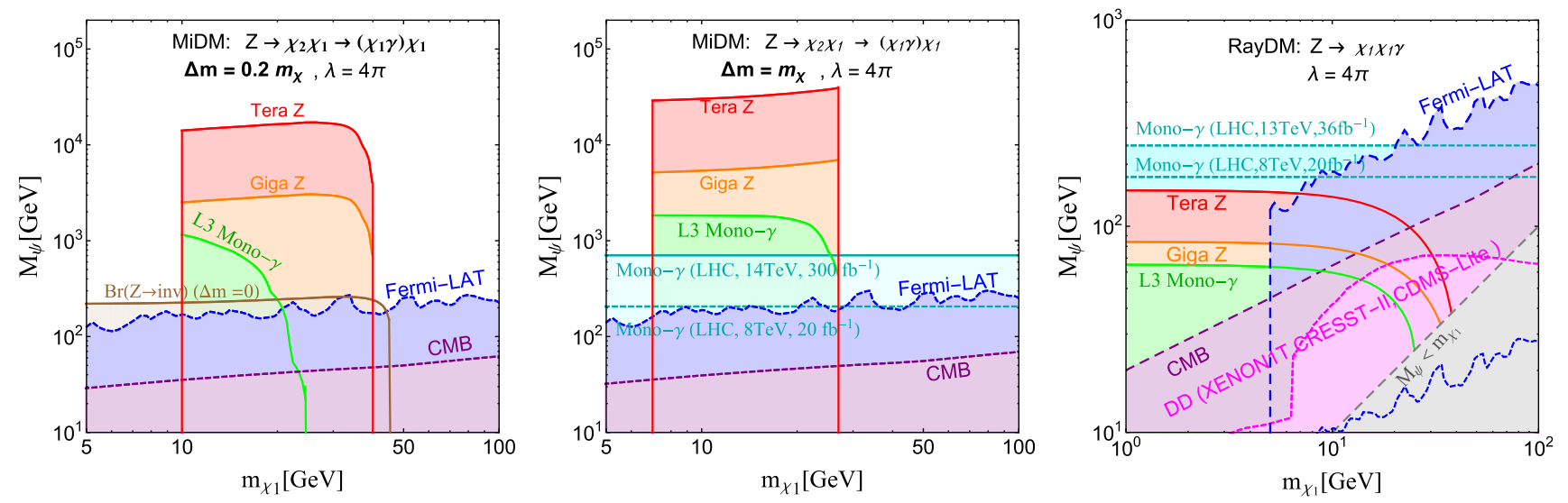

FIG. 6. The 95\% C.L. sensitivity for $M_{\psi}$ from exotic $Z$ decay $Z \rightarrow E+\gamma$, for the MIDM operator in the left (middle) panels with different mass splittings and for the Rayleigh operator in the right panel. The constraints are labeled as Giga $Z$ and Tera $Z$ for a future $Z$ factory with $\lambda=4 \pi$, and the LEP limit from Ref. [64] is shown. We also compare the limits from DM direct detection, indirect detection, monophoton, and monojet searches at the LHC. For RayDM, the gamma-ray constraints from Fermi-LAT and the CMB use a long dashed line for $O_{\text {RayDM }}^{\gamma_{5}}$ and a dashed line for $O_{\text {RayDM }}$. For collider limits, the two operators are similar, and for spin-independent direct detection limits, only $O_{\text {RayDM }}$ is constrained. 
coherence scale $Q_{0}=\sqrt{6}\left(0.3+0.89 A^{1 / 3}\right)^{-1} \mathrm{fm}^{-1}$. The current leading constraints on spin-independent cross sections are XENON1T [69], LUX [70], PANDAX-II [71], and CRESST-II [72] as well as CDMSlite [73]. The limits from direct detection constraints are shown as magenta in Fig. 6. Only $O_{\text {RayDM }}$ is shown as in dashed lines because $O_{\text {RayDM }}^{\gamma_{5}}$ produces the spin-dependent cross section and spin-independent cross section is suppressed.

Existing collider constraints.-Besides DM indirect and direct detection, the MIDM and RayDM operators can also get constraints from monojet and monophoton searches at the LHC and LEP.

The Rayleigh operator $O_{\text {RayDM }}$ has been studied in monophoton, monojet, and mono-V (V stands for vector gauge boson $\mathrm{W}$ and $Z$ ) searches [110]. The limits from monophoton provide the strongest bound and constrain $\Lambda_{\text {RayDM }} \gtrsim 510 \mathrm{GeV}$ at $95 \%$ C.L, for $m_{\chi_{1}} \lesssim 100 \mathrm{GeV}$ from the LHC $8 \mathrm{TeV}$ at $20 \mathrm{fb}^{-1}[111,112]$. Very recently, ATLAS [113] has explored $13 \mathrm{TeV}$ data to search the monophoton signature with integrated luminosity $36 \mathrm{fb}^{-1}$, and it pushes the limit to $\Lambda_{\text {RayDM }} \gtrsim 725 \mathrm{GeV}$. These limits have been integrated in the right panel of Fig. 6 and are denoted "mono- $\gamma$." For $O_{\text {RayDM }}^{\gamma_{5}}$, the limits are similar to $O_{\text {RayDM }}$, and therefore we only show the results for $O_{\text {RayDM }}$.

For the MIDM operator $O_{\text {MIDM }}$, Ref. [109] has studied the limits from the monojet, monophoton, and diphoton searches at the 8 and $14 \mathrm{TeV}$ LHC. For a significant splitting, the authors found the monophoton search [111] is the most stringent, similar to the RayDM operator case. For $m_{\chi_{1}}=10 \mathrm{GeV}$, it requires $\Lambda_{\mathrm{MIDM}} \gtrsim 2400 \mathrm{GeV}$, and the result is roughly unchanged for $m_{\chi_{2}}>20 \mathrm{GeV}$. In the left and middle panels of Fig. 6, we vary DM mass $m_{\chi_{1}}$ from 0 to $40 \mathrm{GeV}$. Since its mass is much smaller than the required photon $p_{T}$ and Missing Transverse Energy (MET), we expect the constraint to be similar to $m_{\chi_{1}}=10 \mathrm{GeV}$. For the monophoton search at the LHC $14 \mathrm{TeV}$ with $300 \mathrm{fb}^{-1}$, the corresponding limit is estimated to be $\Lambda_{\mathrm{MIDM}} \gtrsim$ $8200 \mathrm{GeV}$ [109] and labeled as "mono- $\gamma$ " in Fig. 6.

For the MIDM case, it is interesting to note that, when $m_{\chi_{2}}=m_{\chi_{1}}$, the exotic $Z$ decay $Z \rightarrow E_{\gamma}$ loses its sensitivity at the $Z$ factory and also for the monophoton search at the LHC. The monojet search will be better than the monophoton search in this case. Moreover, Ref. [109] pointed out that actually the invisible decay width measurement of $Z$ can beat the monojet search at the LHC $14 \mathrm{TeV}$ with $3 \mathrm{ab}^{-1}$ integrated luminosity, which suggests $M_{\psi} \gtrsim 226 \mathrm{GeV}$ for $m_{\chi_{1,2}}=10 \mathrm{GeV}$. We have plotted the invisible $Z$ width constraint in panel (a) of Fig. 6.

Given the high center-of-mass energy at the LHC, it can search for the EW charged particles $\psi$ and $\phi$ directly from Drell-Yan production and their subsequent cascade decays [114]. The Drell-Yan search could be more restrictive than mono-object searches, but this conclusion is very model dependent; see Ref. [114]. For example, when $\psi$ and $\phi$ are an $S U(2)_{L}$ singlet, or they decay dominantly to a tau lepton and (or) gauge bosons, the sensitivity from Drell-Yan is very poor, even at the LHC $14 \mathrm{TeV}$ with $300 \mathrm{fb}^{-1}$.

For a monophoton at LEP, the L3 Collaboration has collected data with $137 \mathrm{pb}^{-1}$ at the $Z$ pole, which can limit the BR of exotic decay $Z \rightarrow \gamma E$ down to $1.1 \times 10^{-6}$ if photon energy is greater than $\sim 30 \mathrm{GeV}$ [64]. The OPAL Collaboration has a similar study at the $Z$ pole but with only $40.5 \mathrm{pb}^{-1}$ [65]. There are also many off- $Z$ peak measurements on the single photon final state. The one with $176 \mathrm{pb}^{-1}$ data taken at $189 \mathrm{GeV}$ has been carried out by the L3 Collaboration, which looks for MIDM topology $Z \rightarrow \chi_{2} \chi_{1} \rightarrow\left(\chi_{1} \gamma\right) \chi_{1}$ and bounds the cross section of such topology to be smaller than $0.15-0.4 \mathrm{pb}$ with some dependence on $m_{\chi_{1}}$ and $m_{\chi_{2}}$ [66]. The leading constraint is from L3 measurement at $Z$ pole due to a large resonant cross section, and we label the constraints as "L3 Mono- $\gamma$ " in Fig. 6. We see that this constraint is comparable to the future LHC reach in middle panel of Fig. 6.

\section{Prospects from exotic $Z$ decay}

Exotic $Z$-decay sensitivity.-For exotic $Z$ decay with final state $E \gamma$, we summarize the results of the cascade decay process $Z \rightarrow \chi_{2} \chi_{1} \rightarrow \chi_{1} \chi_{1} \gamma$ from $O_{\mathrm{MIDM}}$ and of the threebody process $Z \rightarrow \chi_{1} \chi_{1} \gamma$ from $O_{\text {RayDM }}$ in Fig. 5 . The limits on such an exotic decay BR are given in Sec. IV. B, and we can calculate the limits for $\Lambda_{\text {MIDM }}$ and $\Lambda_{\text {RayDM }}$ accordingly, then convert them into constraints for $M_{\psi}$ by Eq. (31). The limits are given in Fig. 6 and labeled as "Giga $Z$ " and "Tera Z."

The results of the MIDM operator are presented in the left and middle panels of Fig. 6 . We find exotic $Z$ decay can reach $M_{\psi} \sim \mathcal{O}\left(10^{4}\right) \mathrm{GeV}$, which is much better than monophoton searches at the HL-LHC with $M_{\psi} \sim 10^{3} \mathrm{GeV}$. The production cross sections for $\chi_{2} \chi_{1}$ at the $Z$ factory and LHC both scale as $1 / \Lambda_{\text {MIDM }}^{2}$. However, the cross section at the $Z$ factory benefits from $Z$ resonance compared to the LHC and therefore has larger statistics. Moreover, the $\not E+\gamma$ searches at $Z$ factories have a much cleaner environment than the hadron collider. As a result, exotic $Z$ decay can give the $M_{\psi}$ reach two orders better than the monophoton search at the LHC or HL-LHC. The indirect detection of gamma lines at Fermi-LAT provides a constraint similar to the $8 \mathrm{TeV}$ LHC. The direct detection does not provide any constraint for the MIDM operator because the mass splitting between $\chi_{1}$ and $\chi_{2}$ is too large.

In the right panel of Fig. 6, for the RayDM operator, we find the monophoton search at the LHC can make $M_{\psi}$ reach a few hundreds of $\mathrm{GeV}$, which is better than exotic $Z$ decay with $M_{\psi} \gtrsim 100 \mathrm{GeV}$. The reason is that the cross section for $\chi_{1} \chi_{1} \gamma$ is proportional to $s^{2} / \Lambda_{\mathrm{RayDM}}^{6}$. Since the $Z$ factory has a small center-of-mass energy square, $s \sim m_{Z}^{2}$, it has less sensitivity. The constraint from direct detection is very 
weak because it is a two-loop process. The gamma line constraint from Fermi-LAT is comparable to other constraints and is strongest at $m_{\chi_{1}}$ around $100 \mathrm{GeV}$.

Summary.-We find complementarity between exotic $Z$ decay $Z \rightarrow E_{\gamma}$ at the $Z$ factory and the monojet or monophoton search at the LHC with large mass splitting between $\chi_{1}$ and $\chi_{2}$. For very small mass splitting, the photon from cascade decay $\chi_{2} \rightarrow \chi_{1} \gamma$ becomes very soft, and thus the monophoton and monojet searches via initial state radiation are better. However, invisible $Z$ width measurement can provide a better limit, $M_{\psi} \gtrsim 226 \mathrm{GeV}$. For the MIDM operator, a future $Z$ factory can provide the leading constraints, while for the RayDM operator, the HL-LHC can provide better constraints.

\section{Axionlike particle}

The ALP is a light pseudoscalar which couples to gauge fields via anomalous terms and interacts with fermions with derivatives, $\partial_{\mu} a \bar{\psi} \gamma \gamma^{\mu} \psi$. Its presence is quite generic in UV theories, such as string theory $[30,32,34]$ and supersymmetry [26-28]. It can be a portal connecting dark matter with the Standard Model sector [31], and ultralight ALP is a dark matter candidate by coherent oscillation in the Universe [115-117]. Recently, the dynamics of ALPs in the Universe has also been proposed to solve the Higgs hierarchy problem [118]. For our Z-factory study, we are focusing on the mass range of ALPs from $0.1 \mathrm{GeV}$ to $Z$ boson mass. Although we focus on the case of ALPs, our analysis and results in this section can be applied to the scalar easily.

ALPs can have interactions with Standard Model particle fermions, gauge fields, and Higgs obeying the (discrete-) shift symmetry. Here, we focus on the ALP coupling to the $U(1)_{Y}$ gauge field $B_{\mu}{ }^{4}$,

$$
\mathcal{L}_{\mathrm{ALP}}=\frac{1}{4 \Lambda_{a B B}} a B_{\mu \nu} \tilde{B}^{\mu \nu}
$$

This interaction gives the decay rate of the ALP as

$$
\Gamma(a \rightarrow \gamma \gamma)=\frac{1}{64 \pi} \frac{1}{\Lambda_{a B B}^{2}} \cos \theta_{w}^{4} m_{a}^{3}
$$

and the rate of the $Z$ decay

\footnotetext{
${ }^{4}$ The coupling to fermions is neglected here for simplicity. The ALP coupling to fermions is $c_{f} m_{f} / \Lambda$, where the $c_{f}$ coefficient is model dependent. $a \rightarrow \gamma \gamma$ is the dominant decay channel for very light ALPs, and the decays to fermions are suppressed by $m_{f}^{2} / m_{a}^{2}$ when ALP is significantly heavier than the fermion. If the fermion coupling comes through the gauge field loops, this gets further suppression via the loop effects.
}

$\Gamma(Z \rightarrow \gamma a)=\frac{1}{96 \pi} \frac{1}{\Lambda_{a B B}^{2}} \cos \theta_{w}^{2} \sin \theta_{w}^{2} m_{Z}^{3}\left(1-\frac{m_{a}^{2}}{m_{Z}^{2}}\right)^{3}$.

Depending on the $a \rightarrow \gamma \gamma$ decay length, the analyses are performed in the two separate regimes: one is ALPs decaying inside the detector, and the other is decaying outside the detector. For decay inside the detector, we focus on the prompt search and leave the interesting case of the displaced vertex to future work. For decay outside the detector, the signal is monophoton $+E$. The transverse radius of the detector radius is taken to be $6 \mathrm{~m}$. The decay length of the ALP is computed according to the boost $\gamma_{a}$ of the ALP, $\mathrm{D} \equiv \gamma_{a} c \tau_{a}$, where the $\gamma_{a}=E_{a} / m_{a}$ is the boost and $\tau_{a}=1 / \Gamma_{a}$ is the lifetime of $a$. Since the initial state is a $Z$ boson at rest and the final state is $a \gamma$, the energy $E_{a}$ is fixed by $m_{a}$. D $=6 \mathrm{~m}$ is plotted in Fig. 8 as a dotted black line. Below it, the ALP has a decay length D smaller than $6 \mathrm{~m}$. However, it can still decay outside the detector with a probability of $1-e^{-\mathrm{D} /(6 \mathrm{~m})}$. We account for this probability to rescale the signal events in the detector, which leads to sensitivity below the line. In the prompt decay region, for the high mass axion, the boost of the axion is small, and the dominant channel to search for ALPs is $3 \gamma$. When the mass of the ALP is below $\mathcal{O}(1) \mathrm{GeV}$, the boost of axion makes the two photons from axion decay close enough and cannot be resolved. The $2 \gamma$ search channel is more relevant.

The current constraints for this operator are given by LEP and LHC photon searches. In Fig. 8, the LEP I [119] uses the inclusive diphoton search $e^{+} e^{-} \rightarrow 2 \gamma+X$ covering the small mass region. In the higher mass region, the boost of the axion decreases, and the $3 \gamma$ channel is considered. LEP II and OPAL have $2 \gamma$ and $3 \gamma$ data [120], which are employed to put the bounds on the process, $e^{+} e^{-} \rightarrow \gamma / Z^{\star} \rightarrow a \gamma \rightarrow 2 \gamma+\gamma$. The L3 Collaboration has searched the process $Z \rightarrow a \gamma \rightarrow(\gamma \gamma) \gamma$ at the $Z$ pole, with a limit on the BR of order $10^{-5}$ [67]. The ATLAS $3 \gamma$ and $Z \rightarrow 3 \gamma$ [121,122] search can be translated to the ALP bound as derived in Ref. [123].

For the $E+\gamma$ search, the strongest bound from LEP comes from the L3 Collaboration with $137 \mathrm{pb}^{-1}$ data at the $Z$ pole [64] as discussed in Sec. III C. It can limit the BR of exotic decay $Z \rightarrow \gamma E^{6}$ down to $1.1 \times 10^{-6}$ if photon energy is

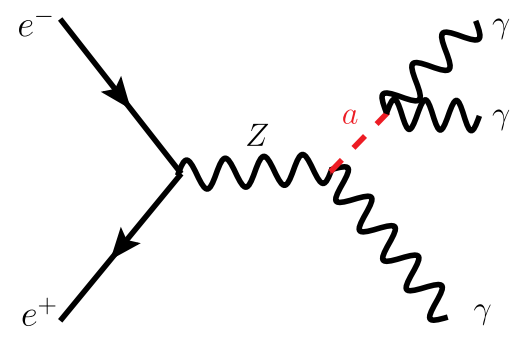

FIG. 7. The Feynman diagram for the exotic $Z$ decay $Z \rightarrow a \gamma \rightarrow(\gamma \gamma) \gamma$. The final state is $3 \gamma$, and in case $m_{a}$ is too small to separate the two photons, the final state is $2 \gamma$. 


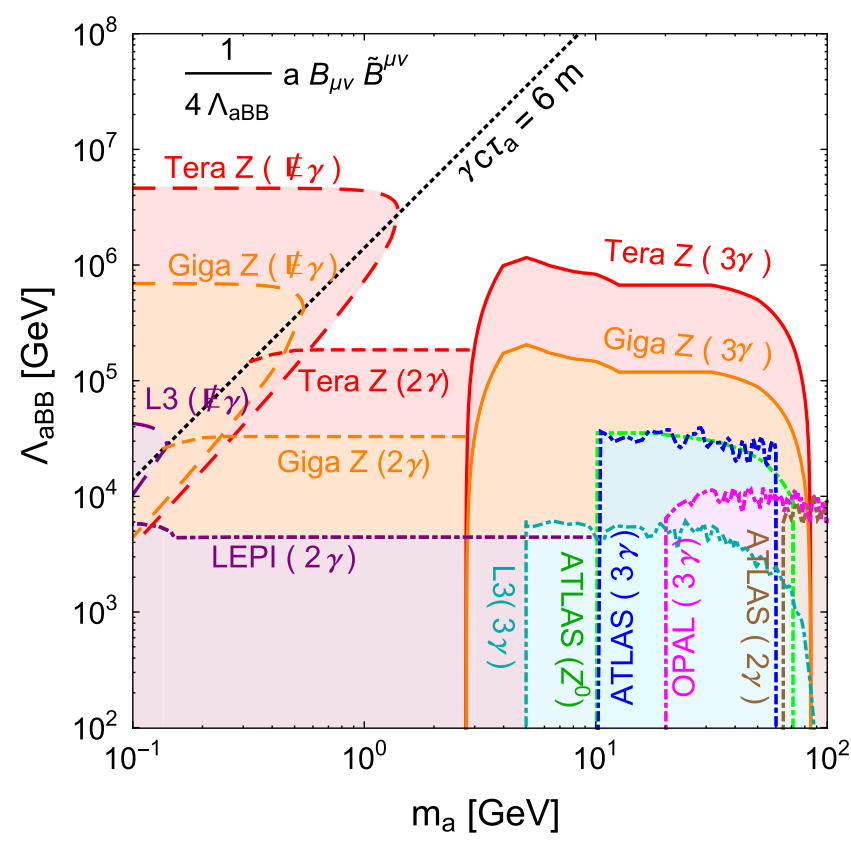

FIG. 8. The limit on $\Lambda_{a \mathrm{BB}}$, ALP coupling to the hypercharge field, from a future $Z$ factory. The limits from the LEP I [119] $\gamma \gamma$ search, LEP II (OPAL) $2 \gamma$ and $3 \gamma$ searches [120], LEP (L3) $3 \gamma$ search at the $Z$ pole [67], ATLAS $3 \gamma$ search, and $Z \rightarrow 3 \gamma$ [121,122] search are translated to limits on $\Lambda_{a \mathrm{BB}}$ following Ref. [123]. There are three types of signals, $Z \rightarrow 2 \gamma, 3 \gamma$, and $E_{\gamma}$, depending on $m_{a}$. In the $E_{\gamma}$ final state where $a$ decay outside the detector, we have set the detector length to be $6 \mathrm{~m}$, and LEP limits on this final state from the L3 Collaboration [64] have been plotted.

greater than $\sim 30 \mathrm{GeV}$. It directly excludes $\Lambda_{\mathrm{aBB}}<4.3 \times 10^{4}$ for $Z \rightarrow E+\gamma$ decay, and we label it as "L3 (E⿱ $\gamma$ )" in Fig. 8.

In the $Z$-decay search, the ALP will give topologies $Z \rightarrow E E+\gamma$ and $Z \rightarrow 3 \gamma, 2 \gamma$, depending on the lifetime and boost of the ALP. We give the Feynman diagram in Fig. 7. $Z$-factory limits on the ALP are given in Fig. 8, which is about 2 orders of magnitude better than the current constraints from the LEP and LHC.

\section{SEARCHING FOR EXOTIC $Z$ DECAYS AT FUTURE $Z$ FACTORIES}

In this section, we make projections for the sensitivity of exotic $Z$-decay searches at future $Z$ factories. Motivated by the previously discussed dark sector models, we classify decay channels by final states, the number of intermediate resonances, and different topologies. In most of the cases, we clarify the connections between the potential models and each topology. As $Z$ is neutral, the final states of its decay can be described as

$$
Z \rightarrow \not E+n_{\gamma} \gamma+n_{\ell^{+} \ell^{-}} \ell^{+} \ell^{-}+n_{\bar{q} q} \bar{q} q .
$$

Since the lepton and quark are charged, they will show up in pairs. The $n$ is referred to as the number of particles or pairs of particles. In our analysis, we choose to consider the number of final state particles to be less than 5. The $E$ can be considered as two particles, since normally it is constituted of two DM particles. It also can be a neutral particle which does not interact with detector and decays outside of it. The final states can be further grouped according to whether they are the decay products of some intermediate resonance. This resonance can be the mother particles for $(\gamma \gamma), \ell^{+} \ell^{-},(\bar{q} q)$, and $E$. The kinematic information of the resonance decay can help us improve the search strategies. The details of classification are given in Table I. The first set of channels has the missing energy in the final states. Since the electron collider has full kinematic information on the initial states, the missing 4-momentum can be fully reconstructed. This is the major advantage of the electron collider compared with the hadron collider in searching for exotic $Z$ decay with missing energy. The second set of channels does not include missing energy. They are pure jet final states $(j j)(j j),(j j)(b b)$, and $(b b)(b b)$ and the threephoton final state $\gamma \gamma \gamma$. They can come from dark sector particle decays, which do not involve dark matter. Due to the cleaner environment of the electron collider, it is better than hadron colliders at measuring pure hadronic final states. For the $j j j j$ final state, since it has a large SM background, we concentrate on the case where it has two resonances. When generating corresponding SM backgrounds, one additional photon is included to count the initial state radiation (ISR). The on-shell intermediate particles should be neutral, since LEP searches have already put severe constraints on charged particles with mass smaller than $m_{Z} / 2$.

In the following subsections, we will discuss the possible models and the sensitivity of each channel at future $Z$ factory. Section IV. A introduces the basic setup and performance for future $Z$ factories at FCC-ee and CEPC and explores the sensitivity of the exotic $Z \mathrm{BR}$ at this future $Z$ factory for different topologies from Secs. IV. A to IV. G. To compare the future $Z$ factory and HL-LHC, Sec. IV. H presents the reach on those exotic $Z$ BRs for the HL-LHC. The summary of this comparison between the future $Z$ factory and HL-LHC is in Fig. 16.

\section{A. Performance of future $Z$ factories}

The exotic $Z$-decay phenomenology at future $Z$ factories at studied in this section. A $Z$-pole run has been considered for both FCC-ee and CEPC [124,125]. Given that the measured cross section of hadronic $Z$ is $30.5 \mathrm{nb}$ [5], the integrated luminosities for Giga $Z\left(10^{9} Z\right)$ and Tera $Z\left(10^{12}\right.$ $Z$ in the plan of FCC-ee) are $22.9 \mathrm{fb}^{-1}$ and $22.9 \mathrm{ab}^{-1}$, respectively.

We simulate the backgrounds and signals in the electron-positron colliders at the $Z$ mass energy using MADGRAPH5_AMC@NLO [126] and analyze them at the parton level. Assuming that the detector performance is similar for different future electron colliders, we follow 
TABLE I. Classification of exotic $Z$-decay channels by particles in final states and number of resonances $\left(n_{\text {res }}\right)$. The $\chi$ and $\chi_{1}$ are fermionic DM, $\chi_{2}$ is an excited state of DM, and $S$ denotes scalar DM. The final state $J$ represents either light flavor jet $j$ or heavy flavor jet $b . A^{\prime}$ is the dark photon, and the $\phi$ is intermediate scalars. The parentheses indicate a resonance in the final states. The details of these models are discussed in the text.

\begin{tabular}{|c|c|c|c|}
\hline Exotic decays & Topologies & $n_{\text {res }}$ & Models \\
\hline$Z \rightarrow \mathscr{E}+\gamma$ & $\begin{aligned} Z \rightarrow & \chi_{1} \chi_{2}, \chi_{2} \rightarrow \chi_{1} \gamma \\
& Z \rightarrow \chi \bar{\chi} \gamma \\
Z \rightarrow a \gamma & \rightarrow(E)^{\prime} \gamma \\
Z \rightarrow A^{\prime} \gamma & \rightarrow(\bar{\chi} \chi) \gamma\end{aligned}$ & $\begin{array}{l}0 \\
0 \\
1 \\
1\end{array}$ & $\begin{array}{l}\text { 1A: } \frac{1}{\Lambda_{1 \mathrm{~A}}} \overline{\chi_{2}} \sigma^{\mu \nu} \chi_{1} B_{\mu \nu} \text { (MIDM) } \\
\text { 1B: } \frac{1}{\Lambda_{1 \mathrm{~B}}^{3}} \bar{\chi} \chi B_{\mu \nu} B^{\mu \nu} \text { (RayDM) } \\
\text { 1C: } \frac{1}{4 \Lambda_{1 C}} a B_{\mu \nu} \tilde{B}^{\mu \nu} \text { (long-lived ALP) } \\
\text { 1D: } \epsilon^{\mu \nu \rho \sigma} A_{\mu}^{\prime} B_{\nu} \partial_{\rho} B_{\sigma} \text { (Wess-Zumino terms) }\end{array}$ \\
\hline$Z \rightarrow E^{t}+\gamma \gamma$ & $\begin{aligned} & Z \rightarrow \phi_{d} A^{\prime}, \phi_{d} \rightarrow(\gamma \gamma), A^{\prime} \rightarrow(\bar{\chi} \chi) \\
& Z \rightarrow \phi_{H} \phi_{A}, \phi_{H} \rightarrow(\gamma \gamma), \phi_{A} \rightarrow(\bar{\chi} \chi) \\
& Z \rightarrow \chi_{2} \chi_{1}, \chi_{2} \rightarrow \chi_{1} \phi, \phi \rightarrow(\gamma \gamma) \\
& Z \rightarrow \chi_{2} \chi_{2}, \chi_{2} \rightarrow \gamma \chi_{1}\end{aligned}$ & $\begin{array}{l}2 \\
2 \\
1 \\
0\end{array}$ & $\begin{array}{l}\text { 2A: Vector portal } \\
\text { 2B: 2HDM extension } \\
\text { 2C: Inelastic DM } \\
\text { 2D: MIDM }\end{array}$ \\
\hline$Z \rightarrow E^{t}+\ell^{+} \ell^{-}$ & $\begin{array}{c}Z \rightarrow \phi_{d} A^{\prime}, A^{\prime} \rightarrow\left(\ell^{+} \ell^{-}\right), \phi_{d} \rightarrow(\bar{\chi} \chi) \\
Z \rightarrow A^{\prime} S S \rightarrow(\ell \ell) S S \\
Z \rightarrow \phi\left(Z^{*} / \gamma^{*}\right) \rightarrow \phi \ell^{+} \ell^{-} \\
Z \rightarrow \chi_{2} \chi_{1} \rightarrow \chi_{1} A^{\prime} \chi_{1} \rightarrow\left(\ell^{+} \ell^{-}\right) E \\
Z \rightarrow \chi_{2} \chi_{1}, \chi_{2} \rightarrow \chi_{1} \ell^{+} \ell^{-} \\
Z \rightarrow \bar{\chi} \chi \ell^{+} \ell^{-}\end{array}$ & $\begin{array}{l}2 \\
1 \\
1 \\
1 \\
0 \\
0\end{array}$ & $\begin{array}{l}\text { 3A: Vector portal } \\
\text { 3B: Vector portal } \\
\text { 3C: Long-lived ALP, Higgs portal } \\
\text { 3D: Vector portal and inelastic DM } \\
\text { 3E: MIDM, supersymmetry } \\
\text { 3F: RayDM, slepton, heavy lepton mixing }\end{array}$ \\
\hline$Z \rightarrow \not t+J J$ & $\begin{array}{c}Z \rightarrow \phi_{d} A^{\prime} \rightarrow(\bar{\chi} \chi)(j j) \\
Z \rightarrow \phi_{d} A^{\prime} \rightarrow(b b)(\bar{\chi} \chi) \\
Z \rightarrow \chi_{2} \chi_{1} \rightarrow b b \chi_{1}+\chi_{1} \rightarrow b b E\end{array}$ & $\begin{array}{l}2 \\
2 \\
0\end{array}$ & $\begin{array}{l}\text { 4A: Vector portal } \\
\text { 4B: Vector portal }+ \text { Higgs portal } \\
\text { 4C: MIDM }\end{array}$ \\
\hline$Z \rightarrow(J J)(J J)$ & $\begin{array}{l}Z \rightarrow \phi_{d} A^{\prime}, \phi_{d} \rightarrow j j, A^{\prime} \rightarrow j j \\
Z \rightarrow \phi_{d} A^{\prime}, \phi_{d} \rightarrow b \bar{b}, A^{\prime} \rightarrow j j \\
Z \rightarrow \phi_{d} A^{\prime}, \phi_{d} \rightarrow b \bar{b}, A^{\prime} \rightarrow b \bar{b}\end{array}$ & $\begin{array}{l}2 \\
2 \\
2\end{array}$ & $\begin{array}{l}\text { 5A: Vector portal }+ \text { Higgs portal } \\
\text { 5B: Vector portal + Higgs portal } \\
\text { 5C: Vector portal }+ \text { Higgs portal }\end{array}$ \\
\hline$Z \rightarrow \gamma \gamma \gamma$ & $Z \rightarrow \phi \gamma \rightarrow(\gamma \gamma) \gamma$ & 1 & 6A: ALP, Higgs portal \\
\hline
\end{tabular}

the detector effects at CEPC [4] and apply the following Gaussian smearing in our analysis:

photon energy resolution: $\frac{\delta E_{\gamma}}{E_{\gamma}}=\frac{0.16}{\sqrt{E_{\gamma} / \mathrm{GeV}}} \oplus 0.01$,

lepton momentum resolution:

$$
\Delta \frac{\mathrm{GeV}}{p_{T}^{\ell}}=2 \times 10^{-5} \oplus \frac{10^{-3} \mathrm{GeV}}{p_{T}^{\ell} \sin \theta},
$$

jet energy resolution: $\frac{\delta E_{j}}{E_{j}}=\frac{0.3}{\sqrt{E_{j} / \mathrm{GeV}}} \oplus 0.02$.

We make conservative assumptions about the tagging efficiency: $80 \%$ for $b$-tagging efficiency, $9 \%$ for the c quark mistagging rate, and $1 \%$ for the light flavor mistagging rate [4]. We also require that all visible particles satisfy $|\eta|<2.3$ ( $\cos \theta<0.98)$. In addition, the photon, lepton, and jet energy should be larger than $10 \mathrm{GeV}$. For events with missing energy, we require $E^{t}>10 \mathrm{GeV}$ as well. Lastly, both the photons and electrons in the final state are separated by $\theta_{i j} \gtrsim 10^{\circ}=0.175 \mathrm{rad}$. The charged leptons normally have better resolution than photons; thus, the separation requirement that we choose here is conservative. For jets, we use a conservative separation requirement $\theta_{i j} \gtrsim 0.4 \mathrm{rad}$ corresponding to $\Delta R \geq 0.4$ at the LHC. ${ }^{5}$ The study for LEP3 (a $240 \mathrm{GeV}$ circular ee collider using the LHC tunnel) with the CMS detector [128] shows the jet angular resolution can be 30 milliradians for energies below $100 \mathrm{GeV}$. The separation requirement for jets at the lepton collider could be optimized due to much less QCD background than the LHC in principal. We leave the optimization for the lepton collider as a future study.

To derive the exclusion limits, the confidential level for the sensitivity calculation adopts Poisson probability [129]. When background event number $B \gg 1$, the significance is about $S / \sqrt{B}$, which is proportional to $\sqrt{L}$, where $L$ is the integrated luminosity. Therefore, the sensitivity reaches of Giga $Z$ and Tera $Z$ differ by about $10^{1.5}$. When background event number $B \ll 1$, the Poisson distribution with zero background assumption leads to a constant limit for the signal. In this case, the exclusion limit is linear to $L$; thus, Giga $Z$ and Tera $Z$ differ by about $10^{3}$. If $B \ll 1$ for Giga $Z$ while $B>1$ for Tera $Z$, the difference of the sensitivity reach is in the range of $10^{1.5}-10^{3}$.

\footnotetext{
${ }^{5}$ For the other separation condition, see Ref. [127].
} 


\section{B. $Z \rightarrow \mathscr{E}+\gamma$}

In this section, we discuss the exotic $Z$ decay with the final state $E^{\prime}+\gamma$. We consider the decay topologies $Z \rightarrow \chi_{2} \chi_{1} \rightarrow \chi_{1} \gamma+\chi_{1}$ and $Z \rightarrow \chi \chi \gamma$, where $\chi$ and $\chi_{1}$ are fermionic DM. $\chi_{2}$ is an excited DM state which decays back to $\chi_{1}$. We also consider two-body decay $Z \rightarrow a \gamma \rightarrow(\mathscr{E}) \gamma$, where $a$ is a pseudoscalar as the missing energy signal if it is stable at the collider scale or it decays to dark matter particles. We denote these three topologies as 1A, 1B, and 1C, respectively, shown in Table I. The UV models for 1A and 1B are the MIDM and RayDM models, while $1 \mathrm{C}$ is motivated by ALPs. The fourth topology, denoted as $1 \mathrm{D}$, is $Z \rightarrow A^{\prime} \gamma \rightarrow(\bar{\chi} \chi) \gamma$. It can come from the Wess-Zumino term $\epsilon^{\mu \nu \rho \sigma} A_{\mu}^{\prime} B_{\nu} \partial_{\rho} B_{\sigma}$ when the dark photons couple to anomalous currents $[130,131,131]$. After integrating by parts, the longitudinal part of $A^{\prime}$ has interaction similar to the topology $1 \mathrm{C}$; thus, the limit on the exotic $Z$ decay BR is similar to $1 \mathrm{C}$.

The SM backgrounds for these final states are mainly $e^{+} e^{-} \rightarrow \gamma \nu \bar{\nu}$. In our simulation, we include one more photon to account for the ISR effect. For $\gamma \nu_{e} \bar{\nu}_{e}$, this process is mediated by either off-shell $W^{ \pm *}$ or off-shell $Z^{*}$, while $\gamma \nu_{\mu} \bar{\nu}_{\mu}$ and $\gamma \nu_{\tau} \bar{\nu}_{\tau}$ are mediated by off-shell $Z^{*}$. In these processes, most of the $\gamma \mathrm{s}$ come from ISR, or internal bremsstrahlung via the t-channel $\mathrm{W}$ boson. The background photons are generally quite soft due to their origin as ISR.

The three models have the different kinematic distributions for the monophoton. For the topology $1 \mathrm{~A}$, $Z \rightarrow \chi_{2}+\chi_{1} \rightarrow \chi_{1} \gamma+\chi_{1}$, the photon energy spectrum has a box shape due to the cascade decay in this process. The minimum and maximum of the photon energy are

$$
\begin{aligned}
& E_{\gamma}^{\max , 1 \mathrm{~A}}=\frac{m_{2}^{2}-m_{1}^{2}}{4 m_{2}^{2}} \frac{s+m_{2}^{2}-m_{1}^{2}+\sqrt{s^{2}+\left(m_{2}^{2}-m_{1}^{2}\right)^{2}-2 s\left(m_{2}^{2}+m_{1}^{2}\right)}}{\sqrt{s}} \\
& E_{\gamma}^{\min , 1 \mathrm{~A}}=\frac{m_{2}^{2}-m_{1}^{2}}{4 m_{2}^{2}} \frac{s+m_{2}^{2}-m_{1}^{2}-\sqrt{s^{2}+\left(m_{2}^{2}-m_{1}^{2}\right)^{2}-2 s\left(m_{2}^{2}+m_{1}^{2}\right)}}{\sqrt{s}} .
\end{aligned}
$$

The distribution of photon energy is flat between $\left[E_{\gamma}^{\min , 1 \mathrm{~A}}, E_{\gamma}^{\max , 1 \mathrm{~A}}\right]$, and the edge of photon energy distribution can be used to determine the mass of DM. Therefore, aside from the preselection cuts, we further impose the cuts below,

$$
1 \mathrm{~A}: E_{\gamma}^{\min , 1 \mathrm{~A}}<E_{\gamma}<E_{\gamma}^{\max , 1 \mathrm{~A}}, \quad m_{\mathrm{inv}} \geq 2 m_{\chi_{1}},
$$

where $m_{\text {inv }}$ is the invariant mass of the missing energy. The second cut comes from momentum conservation that the invariant mass of a set of particles is larger than or equal to the sum of individual masses. According to the recoil mass relation, $E_{\gamma}$ and $m_{\text {inv }}$ are not independent of each other. If we apply the first cut, the second cut is automatically satisfied. Nevertheless, we list the second cut, since this is not redundant in other cases.

For the topology $1 \mathrm{~B}, Z \rightarrow \chi \chi \gamma$, it has a broad distribution in photon energy. The recoil mass $m_{\text {inv }}$ is related to the photon energy $E_{\gamma}$ by

$$
E_{\gamma}=\frac{s-m_{\mathrm{inv}}^{2}}{2 \sqrt{s}} .
$$

In the meantime, the relation $m_{\mathrm{inv}} \geq 2 m_{\chi}$ gives the maximum allowed photon energy

$$
E_{\gamma}^{\max , 1 \mathrm{~B}}=\frac{s-\left(2 m_{\chi}\right)^{2}}{2 \sqrt{s}} .
$$

Thus, in addition to the preselection cut, we impose the following cuts to further suppress the SM background:

$$
1 \mathrm{~B}: \frac{1}{2} E_{\gamma}^{\max , 1 \mathrm{~B}}<E_{\gamma}<E_{\gamma}^{\max , 1 \mathrm{~B}} \text {. }
$$

The lower bound of $E_{\gamma}$ is chosen to keep a significant amount of the signal event and to reject the SM background as much as possible.

For the topology 1C, $Z \rightarrow a \gamma \rightarrow(E) \gamma$, the photon energy spectrum is a delta function with $E_{\gamma}^{2 \mathrm{C}}=$ $\left(s-m_{\phi_{a}}^{2}\right) /(2 \sqrt{s})$. Considering the photon energy $\sim 10 \mathrm{GeV}$, the energy resolution for this photon energy is around 5\% according to Eq. (40). Therefore, we can choose a $2 \mathrm{GeV}$ window on the photon energy,

$$
1 \mathrm{C}: E_{\gamma}^{1 \mathrm{C}}-1 \mathrm{GeV}<E_{\gamma}<E_{\gamma}^{1 \mathrm{C}}+1 \mathrm{GeV} .
$$

After applying the preselection cuts and the specific cuts for the topologies 1A, 1B, and 1C, we obtain the 95\% C.L. exclusion on the exotic $Z$-decay BR in Fig. 9. In panel (a) of Fig. 9 for the topology $1 \mathrm{~A}$, the numbers in each block are $\log _{10}$ (BR) for Tera $Z$ (black) and Giga $Z$ (dark red). It is clear that the sensitivities on the BR for Giga $Z$ and Tera $Z$ differ by a factor of $10^{1.5}$. The reason is the SM background $\gamma \nu \bar{\nu}$ has an event number much larger than 1 for both Tera $Z$ and Giga $Z$; thus, the sensitivity is scaled as $S / \sqrt{B}$. As a result, the sensitivity scales with luminosity as $\sqrt{L}$, so the 


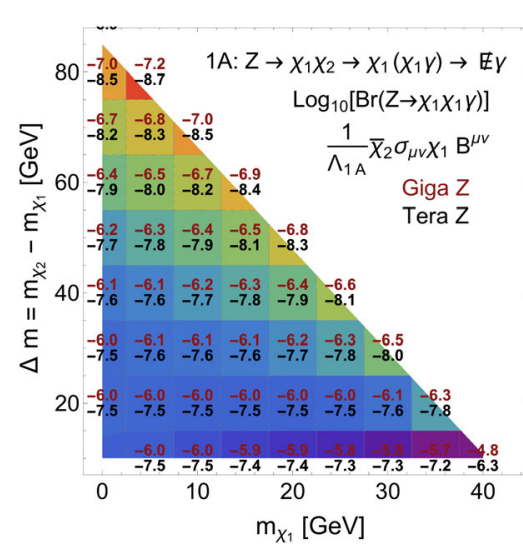

(a)

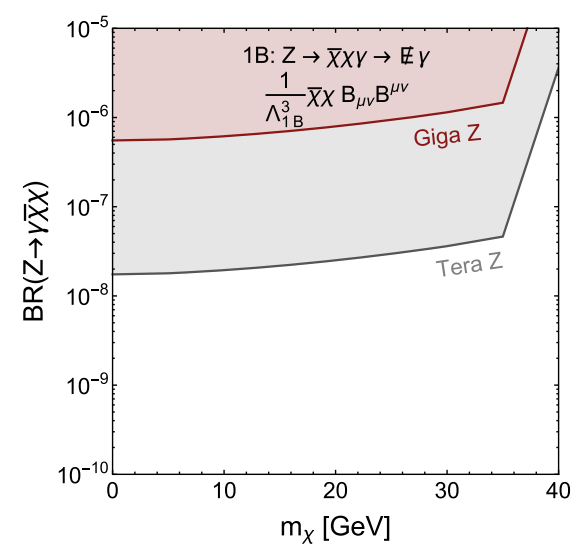

(b)

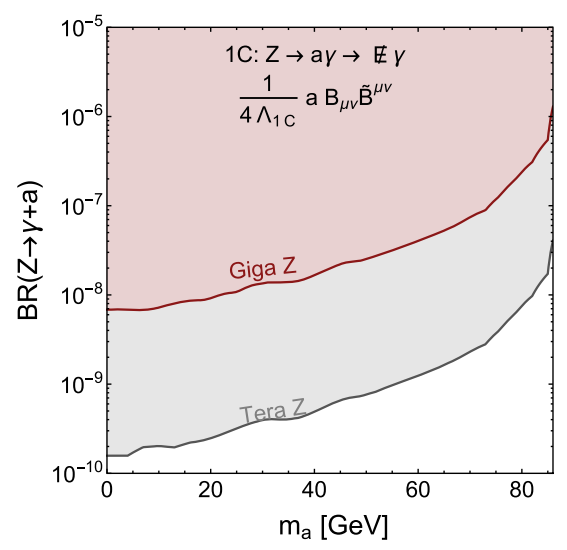

(c)

FIG. 9. The 95\% C.L. exclusion on the exotic $Z$-decay BR for the final state $Z \rightarrow E \gamma$. (a): The decay topology $1 \mathrm{~A}, Z \rightarrow \chi_{2}+\chi_{1} \rightarrow$ $\chi_{1} \gamma+\chi_{1}$ from the MIDM model. The numbers in each block are the sensitivity reach for the exotic $Z$-decay BRs in $\log _{10}$ for Giga $Z$ and Tera $Z$, respectively, while the color mapping is coded for Tera $Z$. (b): The decay topology $1 \mathrm{~B}, Z \rightarrow \chi \chi \gamma$, from the RayDM model. (c): The decay topology $1 \mathrm{C}, Z \rightarrow a \gamma \rightarrow E \gamma$, from the axionlike particle model.

BR sensitivity gets a factor of $10^{1.5}$ increase from Giga $Z$ to Tera $Z$. For Giga $Z$, the limit on the BR falls in the range $10^{-6}-10^{-7}$, while reaches $10^{-7}-10^{-8}$ for Tera $Z$. In panel (b) of Fig. 9 for the topology $1 \mathrm{~B}$, the luminosity scaling between Giga $Z$ and Tera $Z$ is the same as in 1A. The limits on the BR for Giga $Z$ are close to $\sim 10^{-6}$, which is a little bit weaker than $1 \mathrm{~A}$ due to its three-body decay topology. In panel (c) of Fig. 9 for the topology 1C, the luminosity scaling between Giga $Z$ and Tera $Z$ is similar to $1 \mathrm{~A}$ and $1 \mathrm{~B}$. However, the sensitivity on the BR for Giga $Z$ is close to $\sim 10^{-8}$, which is about two orders better than $1 \mathrm{~A}$ and $1 \mathrm{~B}$. The massive resonance in $E^{t}$ implies that the energy of photon is monochromatic, which greatly reduces the SM background.

$$
\text { C. } Z \rightarrow \mathscr{E}+\gamma \gamma
$$

In this section, we focus on the exotic $Z$ decay to the final states $\not E+\gamma \gamma$. The decay topologies can be classified by the number of resonances. The SM background for this final state is coming from $e^{+} e^{-} \rightarrow \gamma \gamma \nu \bar{\nu}$. The general feature of the background is the same as $\gamma \nu \bar{\nu}$, where the photons dominantly come from ISR and tend to be soft.

For topologies with two resonances, the first one is the topology $2 \mathrm{~A}, Z \rightarrow \phi_{d} A^{\prime} \rightarrow(\gamma \gamma)(\bar{\chi} \chi)$, where $A^{\prime}$ is a vector boson which decays into a pair of DM and $\phi_{d}$ is a scalar which decays into a pair of photons. It can be motivated by the vector-portal model in Sec. III. B. 1. The dark Higgs $\phi_{d}$ decays to a diphoton via SM Higgs mixing or by the loop of heavy vectorlike charged particles. The dark photon decays to fermionic DM, which is charged under this $U(1)^{\prime}$.

The second topology with two resonances is the topology 2B. $Z \rightarrow \phi_{A} \phi_{H} \rightarrow(\bar{\chi} \chi)(\gamma \gamma)$, where $\phi_{A}$ and $\phi_{H}$ are $C P$-odd and $C P$-even scalars, respectively. The topology 2B can be motivated well by the two Higgs doublet model
(2HDM). The $C P$-even scalar $\phi_{H}$ is the mixture of $C P$-even scalars in the $2 \mathrm{HDM}$ and can decay to a diphoton via a loop. For the $C P$-odd scalar $\phi_{A}$, decaying to $\bar{\chi} \chi$, one needs to add a singlet $C P$-odd scalar $\phi_{a}$ which couples to $\mathrm{DM}$ via $i \phi_{a} \bar{\chi} \gamma^{5} \chi$. The $\phi_{a}$ can further couple to scalars by $i \phi_{a} H_{1}^{\dagger} H_{2}+$ H.c. [132], where $H_{1,2}$ are the doublet Higgs in the 2HDM. After working out the mass eigenstate, $\phi_{A}$ is the mixture of singlet $C P$-odd scalar $\phi_{a}$ and doublet $C P$-odd scalar in $H_{1,2}$. As a result, it can have the decay topology $Z \rightarrow \phi_{A} \phi_{H}$, and $\phi_{A}$ can further decay to $\bar{\chi} \chi$.

Since the topology $2 \mathrm{~A}$ and $2 \mathrm{~B}$ has the same kinetic feature, the sensitivities to them are similar. Due to the similarity, we take the topology $2 \mathrm{~A}$ as an example. With the presence of two resonances in $\gamma \gamma$ and $\bar{\chi} \chi$, we propose to use the following cuts besides the fiducial selection:

$$
\begin{aligned}
2 \mathrm{~A}:\left|m_{\gamma \gamma}-m_{\phi_{d}}\right| & <2.5 \mathrm{GeV}, \\
\left|m_{i n v}-m_{A^{\prime}}\right| & <2.5 \mathrm{GeV} .
\end{aligned}
$$

Note our invariant mass window cut for the diphoton $\gamma \gamma$ and missing energy $\bar{\chi} \chi$ are conservative. The resolution for diphoton invariant mass is about $0.5 \mathrm{GeV}$ at the LEP [120]. The invariant mass of missing energy is determined by the energy resolution of the diphoton system, which should be smaller than $\lesssim 2 \mathrm{GeV}$ according to Eq. (40).

For the topology with one resonance, we have the topology $2 \mathrm{C}, Z \rightarrow \chi_{2} \chi_{1}$, with the subsequent decays of $\chi_{2} \rightarrow \chi_{1} \phi_{d} \rightarrow \chi_{1}(\gamma \gamma)$, where $\chi_{1,2}$ are the light and heavy $\mathrm{DM}$ and $\phi_{d}$ is a scalar. This topology can be realized by either the MIDM model in Sec. III. 3 or IDM embedded in the vector model in Sec. III. B. 2. Since there is a resonance in $\gamma \gamma$, one can propose the following cuts besides the preselection cuts: 


$$
2 \mathrm{C}:\left|m_{\gamma \gamma}-m_{\phi_{d}}\right|<2.5 \mathrm{GeV}, \quad m_{\mathrm{inv}}>2 m_{\chi_{1}} .
$$

For the topology with 0 resonance, we have 2D, $Z \rightarrow \chi_{2} \chi_{2}$, with the subsequent decay of $\chi_{2} \rightarrow \chi_{1} \gamma$. This topology can be motivated by an extended MIDM model as explained in Sec. III. C. 1. From the event topology, the two photons in the final state have no resonance feature. However, the photon energy distribution has a box shape similar to model 1A. The topology dictates the energy range of both photons,

$$
\begin{aligned}
& E_{\gamma}^{\max , 2 \mathrm{D}}=\frac{m_{2}^{2}-m_{1}^{2}}{4 m_{2}^{2}}\left(\sqrt{s}+\sqrt{s-4 m_{2}^{2}}\right) \\
& E_{\gamma}^{\min , 2 \mathrm{D}}=\frac{m_{2}^{2}-m_{1}^{2}}{4 m_{2}^{2}}\left(\sqrt{s}-\sqrt{s-4 m_{2}^{2}}\right) .
\end{aligned}
$$

Therefore, we propose the following cuts besides the preselection cuts for model $2 \mathrm{D}$,

$$
\text { 2D: } E_{\gamma}^{\max , 2 \mathrm{D}}>E_{\gamma}>E_{\gamma}^{\min , 2 \mathrm{D}}, \quad m_{\mathrm{inv}}>2 m_{\chi_{1}} .
$$

In Fig. 10, we show the 95\% C.L. exclusion on the exotic $Z$-decay BR for the final state $Z \rightarrow E$ r $\gamma$. In panel (a) of Fig. 10 with two resonances and $m_{\chi_{1}}=0 \mathrm{GeV}$, the SM background events are so suppressed that the event number is typically smaller than 1 . As a result, the sensitivity does not scale as $S / \sqrt{B} \sim \sqrt{L}$, but scale as $S / \sqrt{B} \sim L$. This behavior can be seen from panel (a) in Fig. 10 that the BR sensitivity of Giga $Z$ is around $\left[10^{-8.4}, 10^{-6.7}\right]$ and $\left[10^{-11}, 10^{-9.7}\right]$ for Tera $Z$. The sensitivity difference of these two is about $10^{-2}-10^{-3}$. The best sensitivity appears near the region where $m_{A^{\prime}} \geq 10 \mathrm{GeV}$ because of the preselection cut $E_{\text {inv }}>10 \mathrm{GeV}$. The sensitivity gets better when $m_{\phi_{d}}$ becomes large since the photon becomes more energetic and the SM background becomes smaller. In panel (b) of Fig. 10, we assume $m_{\chi_{1}}=0$. With only one resonance, one should expect the sensitivity of figure (b) to be weaker than the sensitivity in figure (a) with two resonances. We do see this point that the sensitivity for figure (a) is better than panel (b) at the same scalar mass $m_{\phi_{d}}=m_{\phi}$ for the Tera $Z$ case but not for Giga $Z$. We have looked into the cut efficiency of signal and background, which explains such behavior. The cut efficiencies for the signal in (a) and (b) are about the same order $\mathcal{O}(0.1-0.8)$. But the panel (b) has a slightly larger efficiency than (a) because in panel (b) the scalar $\phi$ is easier to get a larger energy share by competing with massless $\chi_{1}$, while in figure (a), the scalar $\phi_{d}$ needs to compete with massive $A^{\prime}$. For Figs. 10(a) and 10(b), they have the same SM background. The background efficiencies are $\mathcal{O}\left(10^{-4}\right)$ and $\mathcal{O}\left(10^{-2}\right)$ for (a) and (b), respectively, which shows that the resonance condition for missing energy does help to reduce the SM background. In the Giga $Z$ case, the background event is already smaller than 1 for panel (b); therefore, it has slightly better sensitivity than panel (a) due to higher signal acceptance. For the case of Tera $Z$, the increased luminosity has brought back the need to suppress the SM background; therefore, panel (a) has better sensitivity than (b). In panel (c) of Fig. 10, the limits on the exotic $Z$-decay BR are not as good as panels (a) and (b) because there is no resonance feature in the topology. However, the constraints can still reach $\left[10^{-8.4}, 10^{-7.4}\right]$ for Giga $Z$ and $\left[10^{-10.3}, 10^{-9.2}\right]$ for Tera $Z$.

For the panels (a), (b), and (c) in Fig. 10, one might expect the sensitivity on BR decreases because the number of resonances $n_{\text {res }}$ decreases. This is clearly true when comparing $n_{\text {res }}=1,2$ with $n_{\text {res }}=0$. However, for $n_{\text {res }}=2$ and $n_{\text {res }}=1$, the difference in sensitivity is not very significant, while the sensitivity relies more on the particle mass and the cuts. For example, the best sensitivity for $2 \mathrm{~A}$

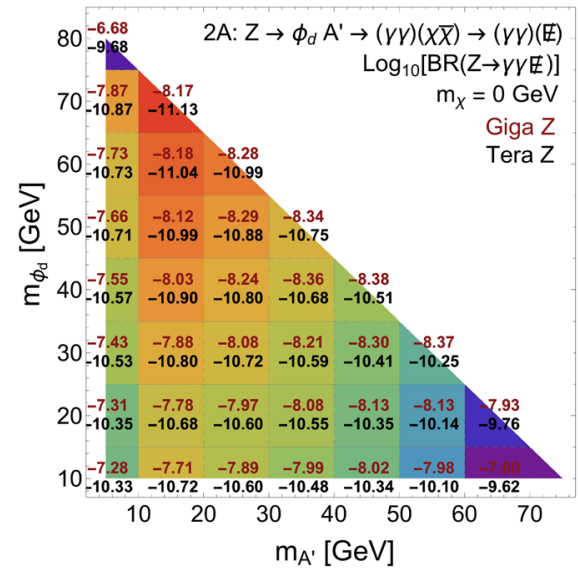

(a)

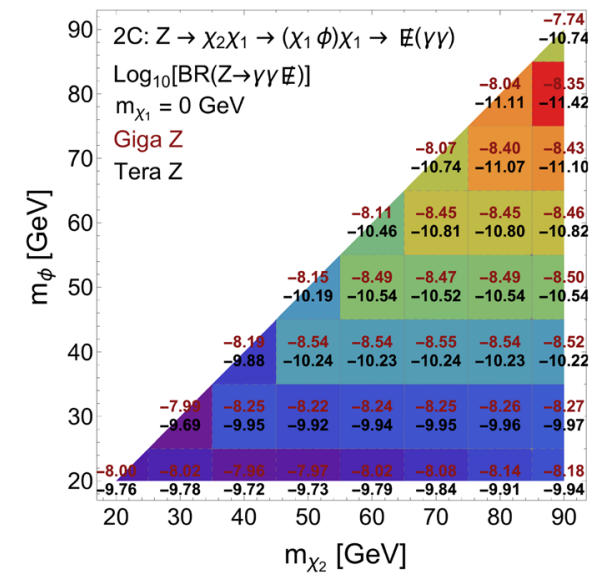

(b)

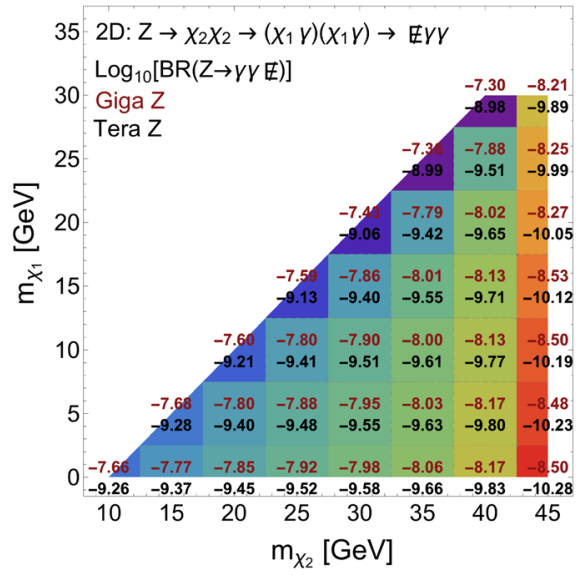

(c)

FIG. 10. The 95\% C.L. exclusion on the exotic $Z$-decay BR for the final state $Z \rightarrow E \gamma \gamma$. The numbers in each block are the sensitivity reaches for the exotic $Z$-decay BR in $\log _{10}$ for Giga $Z$ and Tera $Z$, respectively, and the color coding is based on Tera $Z$. (a): The decay topology $2 \mathrm{~A}, Z \rightarrow \phi_{d} A^{\prime} \rightarrow(\gamma \gamma)(\bar{\chi} \chi)$, from the vector-portal model. (b): The decay topology $2 \mathrm{C}, Z \rightarrow \chi_{2} \chi_{1} \rightarrow \phi \chi_{1} \chi_{1} \rightarrow(\gamma \gamma) E$, from IDM embedded in the vector-portal model. (c): The decay topology $2 \mathrm{D}, Z \rightarrow \chi_{2} \chi_{2} \rightarrow \gamma \gamma \chi_{1} \chi_{1}$, from the MIDM model. 
appears when $m_{A^{\prime}} \sim 15 \mathrm{GeV}$ and $m_{\phi_{d}} \sim 60 \mathrm{GeV}$. The higher the $m_{\phi_{d}}$, the higher the photon energy; however, one should also keep $m_{A^{\prime}}$ large enough to pass the missing energy cut $E>10 \mathrm{GeV}$. The best sensitivity for $2 \mathrm{C}$ appears when $m_{\chi_{2}} \sim 90 \mathrm{GeV}$ and $m_{\phi_{d}} \sim 80 \mathrm{GeV}$ if fixing $m_{\chi_{1}}=0 \mathrm{GeV}$. This high $m_{\phi_{d}}$ mass can guarantee a harder photon spectrum than $2 \mathrm{~A}$. Therefore, even without the resonance cut on $E$, the $\mathrm{SM}$ background of $2 \mathrm{C}$ is similar to that of $2 \mathrm{~A}$, making the sensitivities on the BRs similar.

\section{D. $Z \rightarrow E+\ell^{+} \ell^{-}$}

In this section, we focus on the exotic $Z$ decay to final state $E^{+}+\ell^{+} \ell^{-}$. The SM background for this final state is coming from $\ell^{+} \ell^{-} \bar{\nu} \nu$, mediated by off-shell gauge boson $\gamma^{*}, Z^{*}$, and $W^{*}$. Compared to the ISR photon, the energy spectrum of leptons is harder. And the spectrum of the invariant mass of $\ell^{+} \ell^{-}$is softer than that of $\bar{\nu} \nu$. Given the fact that $\ell$ and $\nu$ share similar kinetic distribution when mediated by $W^{*}$, this can not lead to different invariant masses between $\ell^{+} \ell^{-}$and $\bar{\nu} \nu$. The difference originates from that $\ell^{+} \ell^{-}$can be produced from $\gamma^{*}$ favoring smaller invariant masses, while $\bar{\nu} \nu$ from $Z^{*}$ has a much harder spectrum because small $m_{\text {inv }}$ are suppressed by a factor $m_{\text {inv }}^{4} / m_{Z}^{4}$.

We have listed six topologies with the number of resonances from 2 to 0 in Table I. The event topology with two resonances is $3 \mathrm{~A}, Z \rightarrow \phi_{d} A^{\prime}$, with subsequent decays $A^{\prime} \rightarrow\left(\ell^{+} \ell^{-}\right)$and $\phi_{d} \rightarrow(\bar{\chi} \chi)$. The dark Higgs bremsstrahlung process can be naturally realized by the vector-portal model with a dark Higgs in Sec. III. B. 1.

The topologies with one resonance are 3B, 3C, and 3D. The topology $3 \mathrm{~B}$ is a three-body decay $Z \rightarrow A^{\prime} S^{*} S \rightarrow$ $\left(\ell^{+} \ell^{-}\right) E$, which can be motivated from the vector-portal model with scalar DM in Sec. III. B. 1. The topology $3 \mathrm{C}$ is also a three-body process mediated by an off-shell $Z$ or photon, $Z \rightarrow \phi\left(Z^{*} / \gamma^{*}\right) \rightarrow(E) \ell^{+} \ell^{-}$, where $\phi$ is assumed to decay outside of the detector. It can be motivated by the axionlike particle model in Sec. III. D or Higgs portal model in Sec. III. A where $\phi$ is a singlet scalar which mixes with SM Higgs and can decay to DM pair $\bar{\chi} \chi$. The topology 3D is a two-body cascade decay, $Z \rightarrow \chi_{2} \chi_{1} \rightarrow$ $A^{\prime} \chi_{1}+\chi_{1} \rightarrow\left(\ell^{+} \ell^{-}\right)+E$, which can be motivated by the vector portal and inelastic DM in Sec. III. B. 2.

The topologies without a resonance are $3 \mathrm{E}$ and $3 \mathrm{~F}$. The topology $3 \mathrm{E}$ is a cascade decay $Z \rightarrow \chi_{2} \chi_{1}$, with subsequent decay $\chi_{2} \rightarrow \chi_{1} Z^{*} / \gamma^{*} \rightarrow \chi_{1} \ell^{+} \ell^{-}$, where the last step is a three-body decay. Such a process can be motivated from the MIDM operator in Sec. III. C. 1. The topology $3 \mathrm{~F}$ is a fourbody decay process $Z \rightarrow \bar{\chi} \chi\left(Z^{*} / \gamma^{*}\right) \rightarrow \bar{\chi} \chi \ell^{+} \ell^{-}$, which can be motivated from the RayDM operator in Sec. III. C. 1.

We will study the constraints from exotic $Z$ decay in topologies 3A, 3B, 3E, and 3F. They are chosen to represent different $n_{\text {res }}$ and numbers of particles in the cascade decay, from two body to four body. Besides the same preselection cuts, we propose the following different cuts for different topologies:

$3 \mathrm{~A}:\left|m_{\ell^{+} \ell^{-}}-m_{A^{\prime}}\right|<2.5 \mathrm{GeV}, \quad\left|m_{\mathrm{inv}}-m_{\phi_{d}}\right|<2.5 \mathrm{GeV}$,

3B: $\left|m_{\ell^{+} \ell^{-}}-m_{A^{\prime}}\right|<2.5 \mathrm{GeV}, \quad 2 m_{S}<m_{\mathrm{inv}}<m_{Z}-m_{A^{\prime}}$,

3E: $m_{\ell^{+} \ell^{-}}<m_{\chi_{2}}-m_{\chi_{1}}, \quad m_{\mathrm{inv}}>2 m_{\chi_{1}}$,

3F: $m_{\ell^{+} \ell^{-}}<20 \mathrm{GeV}, \quad m_{\mathrm{inv}}>2 m_{\chi}$.

In Fig. 11, we show the constraints on the exotic $Z$-decay branching ratio $\mathrm{BR}\left(Z \rightarrow E \ell^{+} \ell^{-}\right)$. For Giga $Z$, the topologies with $n_{\text {res }}>0$ will probe the exotic $Z$-decay BR down to $\sim 10^{-8.5}$, while for $n_{\text {res }}=0$, the sensitivity of the can reach $\sim 10^{-8}$. With more resonances, the SM background events are suppressed so much that the event number is typically smaller than 1 . As a result, the sensitivity reaches scales as $L$. For $n_{\text {res }}>0$ in panels (a) and (b) of Fig. 11, the sensitivity on the BR between Giga $Z$ and Tera $Z$ differs by factor of $10^{2} \rightarrow 10^{3}$ due to the small SM background, while for $n_{\text {res }}=0$ in panels (c) and (d), the sensitivity on the BR between Giga $Z$ and Tera $Z$ differs by factor of $\sim 10^{1.5}$, which is a very typical scaling from $S / \sqrt{B} \sim \sqrt{L}$.

\section{E. $Z \rightarrow \mathbb{E}+J \boldsymbol{J}$}

In this section, we focus on the exotic $Z$ decay to the final state $\mathscr{E}+J J$. The $J$ includes both the light flavor jets $j$ and bottom quark jets $b$. The topologies are similar as $Z \rightarrow E^{+}+\ell^{+} \ell^{-}$, and the limits on the exotic $Z$-decay $\mathrm{BR}$ are calculated through the same procedure. The SM background for this final state is dominantly from $\bar{\nu} \nu+J J$, mediated by off-shell gauge bosons $\gamma^{*}, Z^{*}$, and $W^{*}$.

We choose three topologies, $4 \mathrm{~A}, 4 \mathrm{~B}$, and $4 \mathrm{C}$, to study the sensitivity reach of the exotic $Z$-decay BR. The topology $4 \mathrm{~A}$ is $Z \rightarrow \phi_{d} A^{\prime} \rightarrow(\bar{\chi} \chi)(j j)$, and $4 \mathrm{~B}$ is $Z \rightarrow \phi_{d} A^{\prime} \rightarrow(b b)(\bar{\chi} \chi)$. Both topologies can be motivated by the vector-portal model in Sec. III. B. Here, we do not use $\phi_{d} \rightarrow j j$ because Yukawa coupling is a suppressed light quark mass. The last topology $4 \mathrm{C}$ is $Z \rightarrow \chi_{2} \chi_{1} \rightarrow b b \chi_{1}+\chi_{1} \rightarrow b b E$, which can be motivated from the MIDM operator in Sec. III. C. 1 . Besides the fiducial cuts, we propose the following cuts for different topologies:

4A: $\left|m_{j j}-m_{A^{\prime}}\right|<5 \mathrm{GeV}, \quad\left|m_{\mathrm{inv}}-m_{\phi_{d}}\right|<5 \mathrm{GeV}$,

4B: $\left|m_{b b}-m_{\phi_{d}}\right|<5 \mathrm{GeV}, \quad\left|m_{\mathrm{inv}}-m_{A^{\prime}}\right|<5 \mathrm{GeV}$,

4C: $2 m_{b}<m_{b b}<m_{\chi_{2}}-m_{\chi_{1}}, \quad m_{\mathrm{inv}}>2 m_{\chi_{1}}$. 


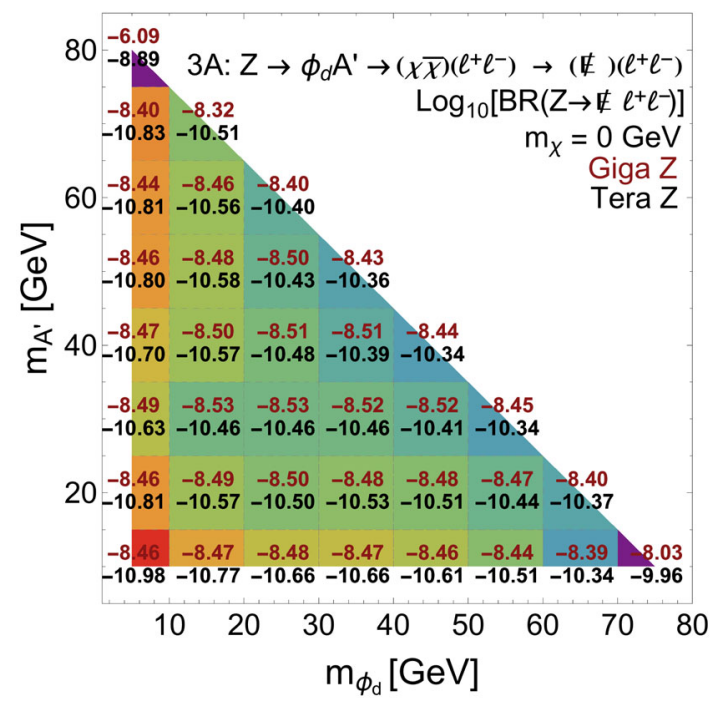

(a)

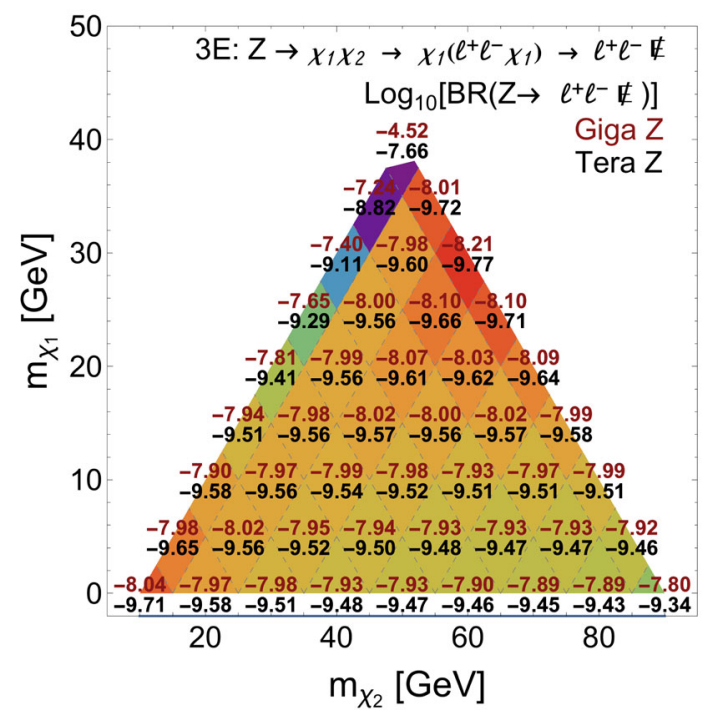

(c)

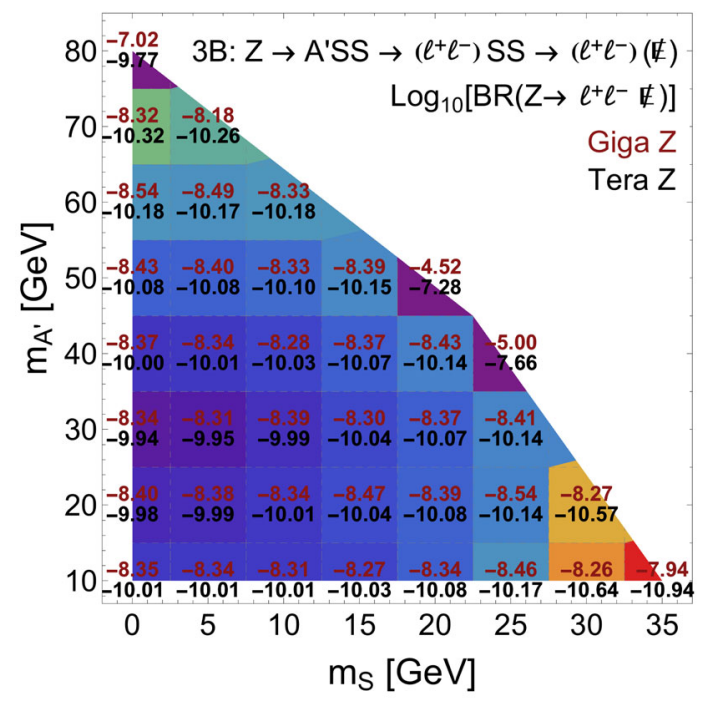

(b)

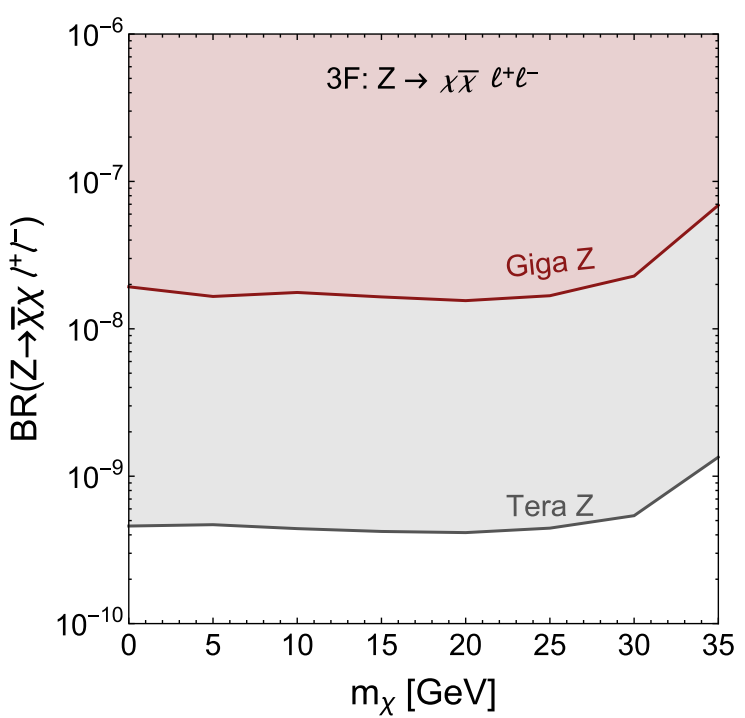

(d)

FIG. 11. The 95\% C.L. exclusion on the exotic $Z$-decay BR for the final state $Z \rightarrow E \ell^{+} \ell^{-}$. The numbers in each block are the sensitivity reach for the exotic $Z$-decay BR in $\log _{10}$ for Giga $Z$ and Tera $Z$, respectively, while the color mapping is coded for Tera $Z$. (a): The decay topology $3 \mathrm{~A}, Z \rightarrow \phi_{d} A^{\prime} \rightarrow(\bar{\chi} \chi)\left(\ell^{+} \ell^{-}\right)$, from the vector-portal model. The numbers in each block are reaches for the exotic $Z$-decay BR in $\log _{10}$ for Giga $Z$ and Tera $Z$. (b): The decay topology $3 \mathrm{~B}$, three-body decay, $Z \rightarrow A^{\prime} S^{*} S \rightarrow\left(\ell^{+} \ell^{-}\right) E^{\star}$, from the vectorportal model with scalar DM $S$. (c): The decay topology $3 \mathrm{E}$, a cascade decay $Z \rightarrow \chi_{2} \chi_{1}$, with subsequent decay $\chi_{2} \rightarrow \chi_{1} Z^{*} / \gamma^{*} \rightarrow$ $\chi_{1} \ell^{+} \ell^{-}$motivated by the MIDM operator. (d): The decay topology $3 \mathrm{~F}$, a four-body decay process, $Z \rightarrow \bar{\chi} \chi\left(Z^{*} / \gamma^{*}\right) \rightarrow \bar{\chi} \chi \ell^{+} \ell^{-}$, which can be motivated from the RayDM operator.

In Fig. 12, we show the constraints on the exotic Z-decay branching ratio $\mathrm{BR}(Z \rightarrow \not E J J)$. For Giga $Z$, the exotic $Z$ decay $\mathrm{BR}$ can be probed down to $10^{-7}-10^{-8}$, while the sensitivity of Tera $Z$ is generally better by factor of $\sim 10^{1.5}$ compared to Giga $Z$. Comparing the BR sensitivity of $4 \mathrm{~A}$ and $4 \mathrm{~B}$, we see that the difference between the light flavor jet $j$ and heavy flavor jet $b$ is not large. One might expect the sensitivity of $E(b \bar{b})$ to be better than $E(j j)$, due to smaller SM background. However, the topologies $4 \mathrm{~A}$ and
4B are not the same, because in $4 \mathrm{~A}$ the jets come from $A^{\prime}$ while in $4 \mathrm{~B}$ the $b$ jets come from $\phi_{d}$.

$$
\text { F. } \boldsymbol{Z} \rightarrow(\boldsymbol{J} \boldsymbol{J})(\boldsymbol{J} \boldsymbol{J})
$$

In this section, we focus on the exotic $Z$ decay to the final state $(J J)+(J J)$. Note that we only discuss the cases where there are two jet resonances in the final states. The SM background for this final state is mostly from the electroweak process, mediated by off-shell gauge bosons $\gamma^{*}, Z^{*}$, and $W^{*}$. 


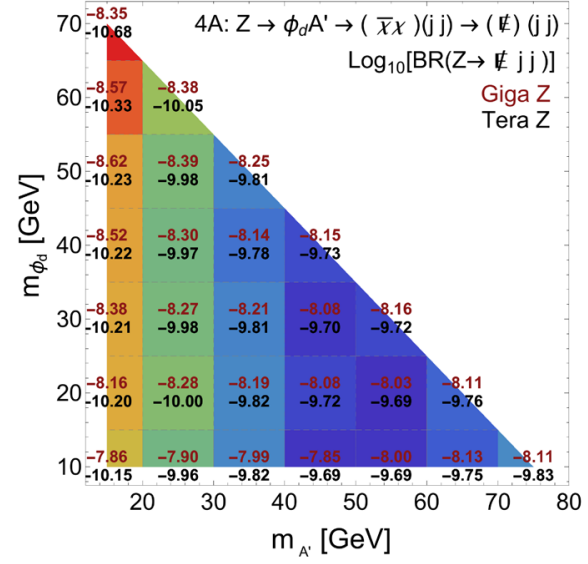

(a)

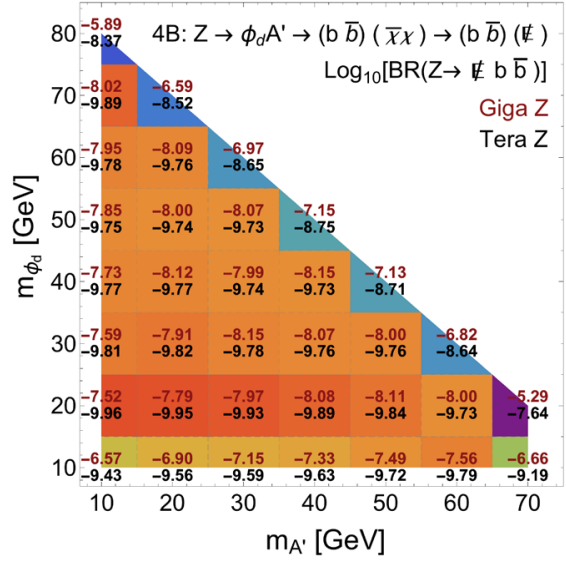

(b)

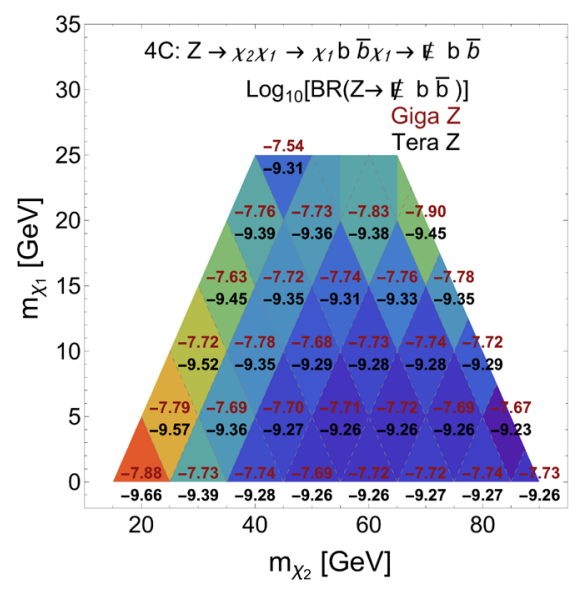

(c)

FIG. 12. The 95\% C.L. exclusion on the exotic $Z$-decay BR for the final state $Z \rightarrow E J J$, where $J$ includes both the light flavor jet $j$ and bottom quark jet $b$. The numbers in each block are the sensitivity reach for the exotic $Z$-decay BR in $\log _{10}$ for Giga $Z$ and Tera $Z$, respectively, while the color mapping is coded for Tera $Z$. (a): The decay topology $4 \mathrm{~A}, Z \rightarrow \phi_{d} A^{\prime} \rightarrow(\bar{\chi} \chi)(j j)$, from the vector-portal model. The numbers in each block are reaches for the exotic $Z$-decay BR in $\log _{10}$ for Giga $Z$ and Tera $Z$. (b): The decay topology 4B, $Z \rightarrow \phi_{d} A^{\prime} \rightarrow(b b)(\bar{\chi} \chi)$, from the vector-portal model. (c): The decay topology $4 \mathrm{C}, \quad Z \rightarrow \chi_{2} \chi_{1} \rightarrow \chi_{1} b b \chi_{1} \rightarrow E b b$, from the MIDM model.

In Table I, we have listed the topologies. 5A could be motivated from Higgs bremsstrahlung in the vectorand scalar-portal models. We will choose the topology $5 \mathrm{~A}$ to illustrate the sensitivity to the $\mathrm{BR}$ of the $(J J)+(J J)$ final state. We divide the final states with three combinations, $(j j)+(j j),(j j)+(b b)$, and $(b b)+(b b)$, where the last two are denoted as $5 \mathrm{~B}$ and $5 \mathrm{C}$. There could be other topologies like $Z \rightarrow$ $\phi_{A} \phi_{H} \rightarrow(J J)(J J)$ from the 2HDM, but the topology and kinematics are similar; therefore, their sensitivity should be similar to $5 \mathrm{~A}$. Beside the preselection cuts, we add the following similar cuts for the topologies $5 \mathrm{~A}$, $5 \mathrm{~B}$, and $5 \mathrm{C}$ :

$$
\begin{aligned}
& \text { 5A: }\left|m_{j j}-m_{A^{\prime}}\right|<5 \mathrm{GeV}, \quad\left|m_{j j}-m_{\phi_{d}}\right|<5 \mathrm{GeV} \\
& \text { 5B: }\left|m_{j j}-m_{A^{\prime}}\right|<5 \mathrm{GeV}, \quad\left|m_{b b}-m_{\phi_{d}}\right|<5 \mathrm{GeV} \\
& \text { 5C: }\left|m_{b b}-m_{A^{\prime}}\right|<5 \mathrm{GeV}, \quad\left|m_{b b}-m_{\phi_{d}}\right|<5 \mathrm{GeV} \text {. }
\end{aligned}
$$

The $\chi^{2}$ method is employed to determine which pair of jets is from $A^{\prime}$ decay or $\phi_{d}$ decay. The mass window that we take is conservative. For example, at $E_{j}=40 \mathrm{GeV}$, the jet energy resolution is about $5 \%$, leading to $\Delta E_{j}=2 \mathrm{GeV}$ from Eq. (42).

In Fig. 13, we show the constraints on the exotic $Z$-decay branching ratio $\mathrm{BR}(Z \rightarrow(J J)(J J))$. For Giga $Z$, the exotic
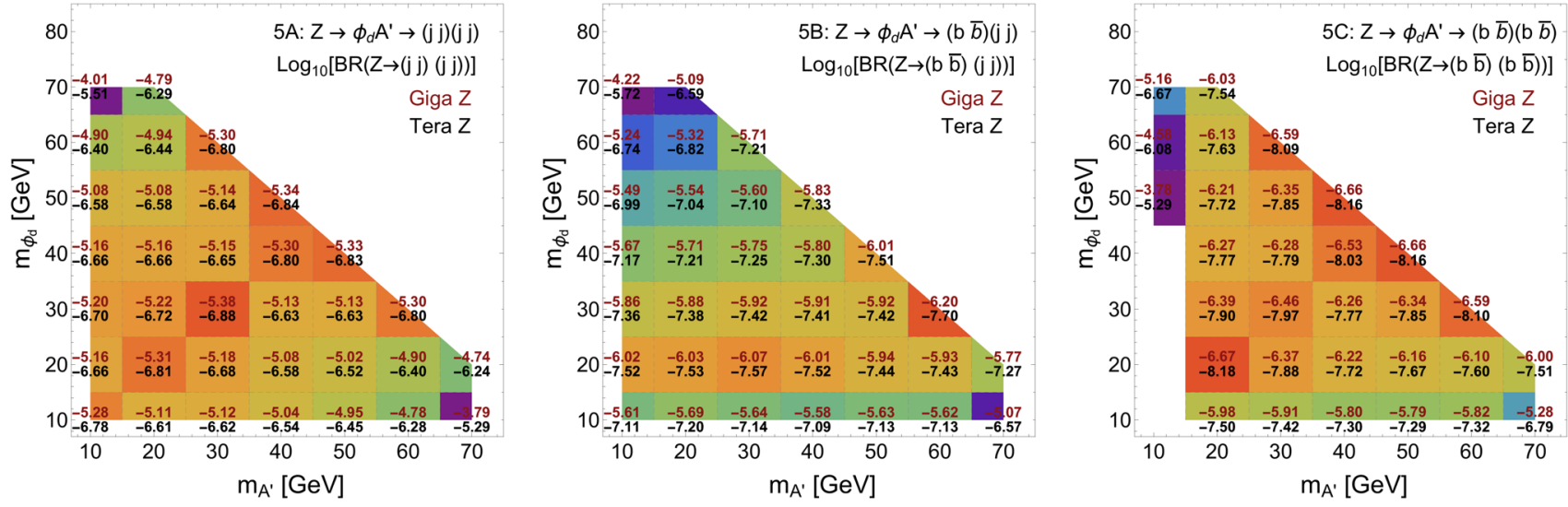

FIG. 13. The $95 \%$ C.L. sensitivity for exotic $Z$ decay $Z \rightarrow(J J)(J J)$, where $J$ could be light flavor jet or $b$ jet. The numbers in each block are the sensitivity reach for the exotic $Z$-decay BR in $\log _{10}$ for Giga $Z$ and Tera $Z$, respectively, while the color mapping is coded for Tera $Z$. The decay process is $Z \rightarrow \phi_{d} A^{\prime}$ with subsequent decays $\phi_{d} \rightarrow j j$ and $A^{\prime} \rightarrow j j$. We show three combinations, $(j j)(j j)$, $(j j)(b b)$, and $(b b)(b b)$, in the figure. 
$Z$-decay BR can be probed down to $\sim 10^{-5}$ for the $(j j)(j j)$ final state, $\sim 10^{-6}$ for $(j j)(b b)$ and $10^{-6.5}$ for $(b b)(b b)$. The sensitivity of Tera $Z$ is generally better by factor of $\sim 10^{1.5}$ compared to Giga $Z$, from the integrated luminosity scaling $S / \sqrt{B} \approx \sqrt{L}$. It is clear that the sensitivity for the heavy flavor jet is slightly better than the light flavor jet. This is because the heavy flavor jet has fewer SM background events, by a factor of $N_{f}^{1 / 2} \approx 10^{0.5}$, where $N_{f}$ is the number of flavor in jets.

\section{G. $Z \rightarrow \gamma \gamma \gamma$}

In this section, we discuss the exotic $Z$ decay to final state $(\gamma \gamma) \gamma$. The SM background for this final state $\gamma \gamma \gamma$ is dominated by the QED process $e^{+} e^{-} \rightarrow \gamma \gamma$ with an extra $\gamma$ from initial state radiation; therefore, the photon energy generally tends to be soft. The signal topology $6 \mathrm{~A}$ in Table I, $Z \rightarrow \phi \gamma \rightarrow(\gamma \gamma) \gamma$, could be motivated from an axionlike particle or from the Higgs portal scalar, which can decay to $\gamma \gamma$ from the top loop. We take the axionlike particle as an example in Fig. 14, and the result should also apply to the Higgs portal scalar. Besides the preselection cuts, we propose the following cuts for topology 6A,

$$
6 \mathrm{~A}:\left|m_{\gamma \gamma}-m_{\phi}\right|<1 \mathrm{GeV}, \quad\left|E_{\gamma}^{3 \mathrm{rd}}-E_{\gamma}^{6 \mathrm{~A}}\right|<1 \mathrm{GeV},
$$

where $E_{\gamma}^{6 \mathrm{~A}}=\left(s-m_{\phi}^{2}\right) /(2 \sqrt{s})$. We use the $\chi^{2}$ method to determine the pair of photons from $\phi$ decay and single out the third photon. The energy of the third photon $E_{\gamma}^{3 \text { rd }}$ is very close to $E_{\gamma}^{6 \mathrm{~A}}$; therefore, we add an energy window cut. In Fig. 14, we see the sensitivity on the $\mathrm{BR}$ for exotic $Z$ decay for topology $6 \mathrm{~A}$ can reach $\sim 10^{-7}$ for Giga $Z$ and $3 \times 10^{-9}$

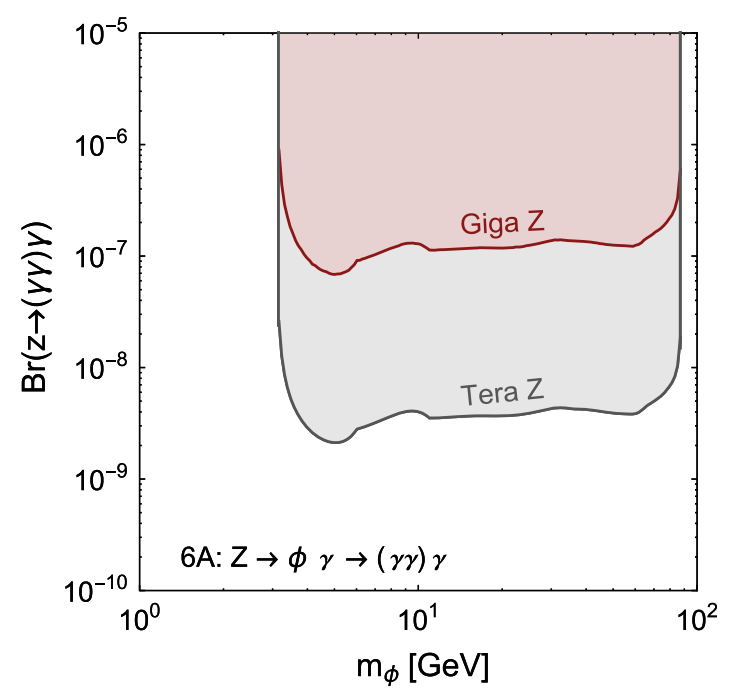

FIG. 14. The $95 \%$ C.L. exclusion for exotic $Z$-decay topology $6 \mathrm{~A}, Z \rightarrow \phi \gamma \rightarrow(\gamma \gamma) \gamma$. The Giga- (Tera-) $Z$ exclusion region is shown in dark red (gray). The $m_{\phi}<2 \mathrm{GeV}$ region is not limited due to photon separation failure but can be constrained by $\gamma \gamma$ search; see Fig. 8. for Tera $Z$. For $m_{\phi}<2 \mathrm{GeV}$, it is hard to separate the two photons from $\phi$ decay, and the signal efficiency goes to zero. Instead of three photons in the final state, one could look for two photons because the photons from $m_{\phi}$ cannot be distinguished and therefore cover this mass range as in Fig. 8.

\section{H. Sensitivity reach of the HL-LHC}

The HL-LHC $\left(3 \mathrm{ab}^{-1}\right)$ also produces a lot of $Z \mathrm{~s}$, which can be sensitive to some of the exotic $Z$-decay modes. In this section, we would like to study the sensitivities for the HL-LHC and compare its reaches with the ones from $Z$ factories. A full-fledged study with realistic detector simulations is beyond the scope of this paper. Instead, we perform simplified simulations aiming to gain an order of magnitude estimation. As we will see, the capabilities of the HL-LHC and $Z$ factories are very different. Our approach is sufficient to highlight the relative strengths of the two experiments. For each topology, we only pick up one benchmark parameter (zero for DM mass and $40 \mathrm{GeV}$ for other new physics mediated particles) to set the HLLHC sensitivity and do not scan the parameter spaces of models because the cut efficiency is not strongly depending on the mass. We have chosen the benchmark mass parameters to give the most energetic $Z$-decay products. In addition, we do not consider fake photons from QCD, which will significantly reduce the HL-LHC sensitivity. In this sense, our projection for the HL-LHC should be considered optimistic. In order to suppress the huge QCD background and avoid prescaling, we search $Z$ production in association with a high $p_{T}$ jet or high $p_{T}$ photon. For all the visible particles, we require $|\eta|<2.5$.

$$
\text { 1. } Z \rightarrow \gamma+E^{h}
$$

For exotic decay $Z \rightarrow \gamma+E$, we generate a $j Z$ event with the MAGDRAPH5 at the $13 \mathrm{TeV} \mathrm{LHC}$ and require the jet to be $p_{T}^{j_{1}}>60 \mathrm{GeV}$ to make $Z$ have enough $p_{T}$ to produce the energetic photon and large enough $\mathscr{E}$ to suppress the SM background. Specifically, we require $E_{T}>50 \mathrm{GeV}$ and $p_{T}^{\gamma}>20 \mathrm{GeV}$ together with $p_{T}^{j_{1}}>60 \mathrm{GeV}$ as the basic cuts. After the parton-level event generation, it is passed to PyTHIA6.4 [133] for showering and hadronization and to DELPHES3.2 [134] for detector simulation. In the detector, missing energy could come from the jet reconstruction due to jet energy resolution and uncertainty. Therefore, we include the SM background $j \gamma$ and irreducible SM background $j \gamma \nu \bar{\nu}$. We list the cross sections after basic cuts for signal $j Z \rightarrow j+\gamma+E^{t}$ and each SM background in Table II in the column labeled with " $\sigma_{\text {basic }}$."

To further optimize the signal, we make the differential distribution for kinetic variables $p_{T}^{j_{1}}, E_{T}^{\prime}$ and $p_{T}^{\gamma}$ in Fig. 15. We compare the distribution of SM background $j \gamma$ and $j \gamma \nu \bar{\nu}$ with signal $j Z$ with exotic $Z$-decay topologies $1 \mathrm{~A}, 1 \mathrm{~B}$, and 1C. Based on Fig. 15, we further impose the following cuts: 
TABLE II. The exotic $Z$-decay final states are listed for both SM backgrounds and signals. The " $\sigma_{\text {basic }}$ " column gives the cross section after basic cuts, and the $\epsilon$ gives the cut efficiency for the further optimized cuts. The above cut efficiencies do not including the $b$-tagging efficiency. In the final sensitivity calculation, we use the $b$-tagging efficiency 0.7 and mistagged efficiency 0.015 [135] to reweight the

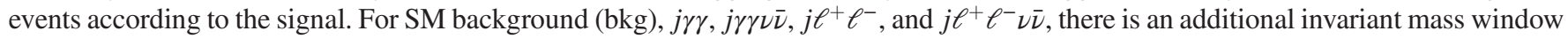
cut for $\gamma \gamma$ or $\ell^{+} \ell^{-}$, which should multiply the efficiency given in parentheses $(\times())$. This additional efficiency is given as a range because the mass window changes with the mediator mass in the signal topology. Such a change is indicated by the light brown shaded region for the HL-LHC in Fig. 16.

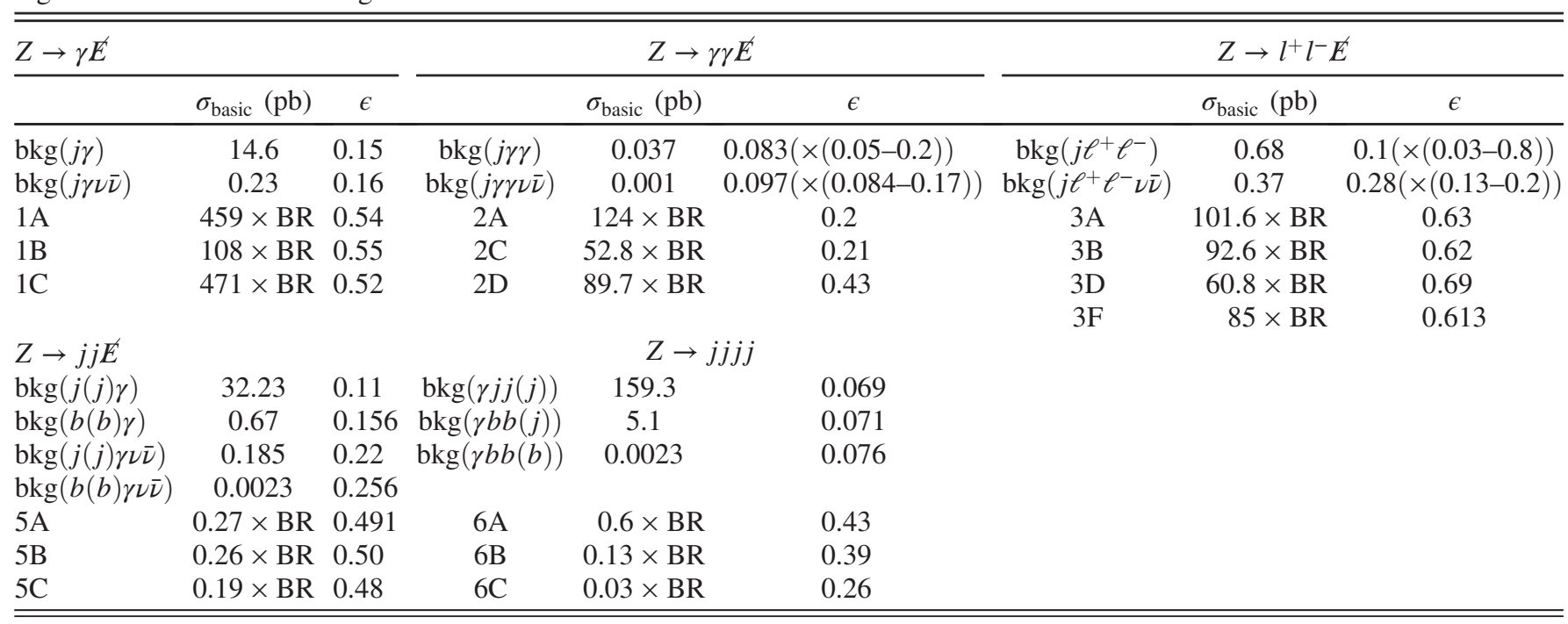

$$
\mathscr{E}_{T}<100 \mathrm{GeV}, \quad p_{T}^{\gamma}<150 \mathrm{GeV}
$$

We do not use additional cuts on $p_{T}^{j_{1}}$ because the distributions of the SM background and signal are quite similar. After applying the above cuts, we list the corresponding cut efficiency in Table II in the column labeled "e." For the HL-LHC $\left(3 \mathrm{ab}^{-1}\right)$, we can reach the sensitivity for the exotic Z-decay BRs of $5.6 \times 10^{-6}, 2.3 \times 10^{-5}$, and $5.76 \times 10^{-6}$ for signal topologies $1 \mathrm{~A}, 1 \mathrm{~B}$, and $1 \mathrm{C}$. The sensitivities for the HL-LHC for each topology are given in the summary plot, Fig. 16.

\section{2. $Z \rightarrow \gamma \gamma+E$}

The SM background we consider is $j \gamma \gamma$ with $E$ from misreconstruction and irreducible $j \gamma \gamma \nu \bar{\nu}$. The basic cuts are $p_{T}^{j}>60 \mathrm{GeV}, E_{T}>50 \mathrm{GeV}$, and two photons with $p_{T}^{\gamma}>20 \mathrm{GeV}$. The cross sections after cuts for the signal and SM background are again listed in Table II. We use the following cuts to further optimize our signal:

$$
\begin{aligned}
p_{T}^{j_{1}} & >80 \mathrm{GeV}, \quad 50 \mathrm{GeV}<\ell_{T}<100 \mathrm{GeV}, \\
40 \mathrm{GeV} & <p_{T}^{\gamma_{1}}<100 \mathrm{GeV} .
\end{aligned}
$$

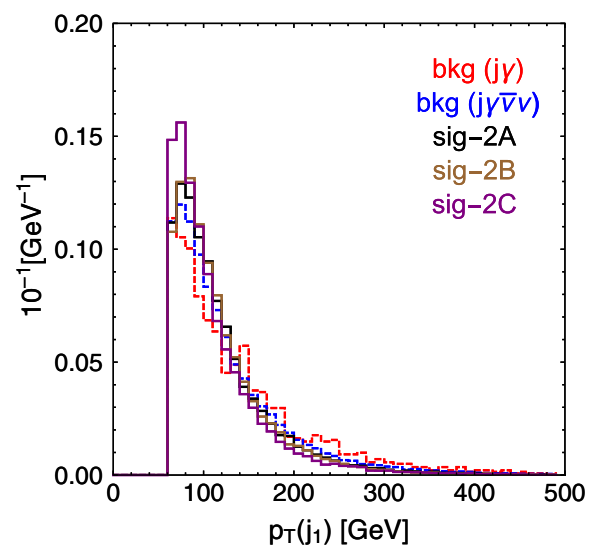

(a)

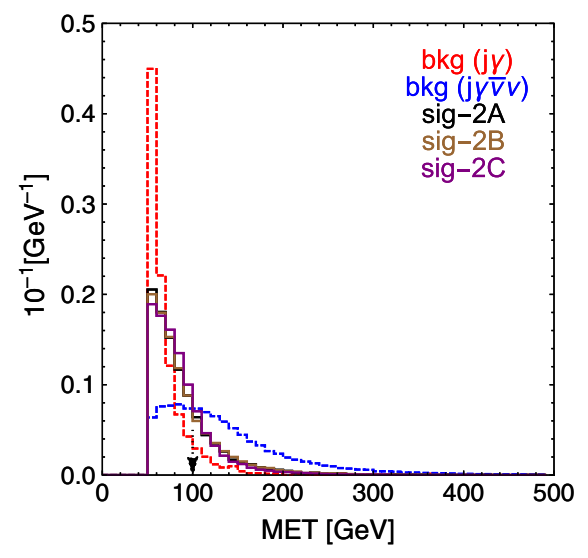

(b)

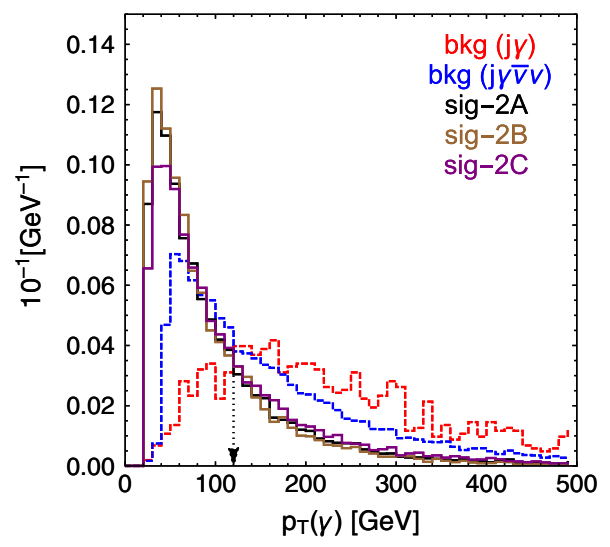

(c)

FIG. 15. The normalized event distributions for kinematic variables $p_{T}^{j_{1}}, p_{T}^{\gamma}$, and $\mathscr{E}_{T}$ for signal $j Z \rightarrow j+\gamma+\mathscr{E}$ and the corresponding SM background. The distributions have been normalized to 1 . 


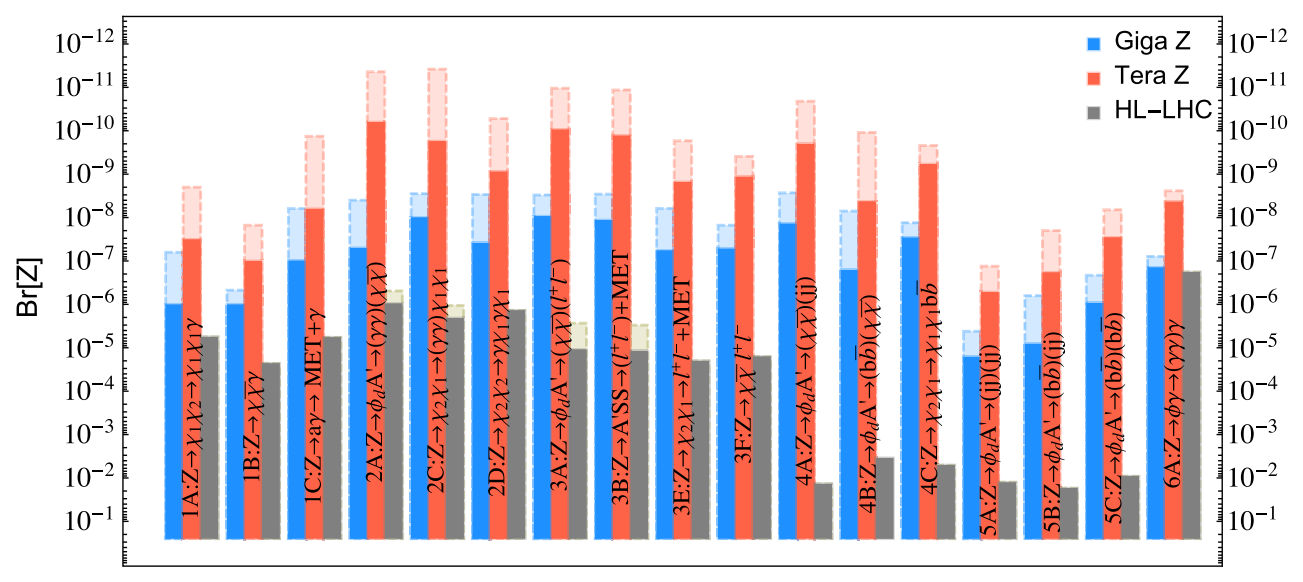

FIG. 16. The sensitivity reach for the BR for various exotic $Z$-decay topologies at the future $Z$ factory (Giga $Z$ and Tera $Z$ ) and the HL-LHC at $13 \mathrm{TeV}$ with $\mathcal{L}=3 \mathrm{ab}^{-1}$. The BR sensitivity generally depends on the model parameter, for example, the mediator mass and dark matter mass. The dark colored region with the solid line as a boundary indicates the worst reach for the topology, while the lighter region with the dashed line indicates the best reach. For the HL-LHC, we add the light shaded region for the topologies $2 \mathrm{~A}, 2 \mathrm{C}, 3 \mathrm{~A}$, and 3B to indicate the effect of an invariant mass window cut for the diphoton and dilepton. For the topology 6A, the HL-LHC limit is obtained by rescaling the ATLAS study at the $8 \mathrm{TeV}$ LHC [122] with $\mathcal{L}=20 \mathrm{fb}^{-1}$.

The cut efficiencies for the signal and SM background are listed in Table II. For topologies 2A and 2C, we can make an additional $5 \mathrm{GeV}$ window cut on the invariant mass of the diphoton to suppress the SM background, while the signal remains unaffected. The corresponding efficiency is listed in parentheses in the $\epsilon$ column in Table II. It is a range for the SM background due to the change of mediator mass. For the HL-LHC $\left(3 a b^{-1}\right)$, the future sensitivity reaches for exotic Z-decay topologies $2 \mathrm{~A}, 2 \mathrm{C}$, and $2 \mathrm{D}$ are $(5-10) \times 10^{-7},(1-2) \times 10^{-6}$, and $1.4 \times 10^{-6}$, respectively, and have been plotted in Fig. 16. The sensitivity range for the topologies $2 \mathrm{~A}$ and $2 \mathrm{C}$ has been indicated by the light brown shaded region.

$$
\text { 3. } Z \rightarrow \boldsymbol{\ell}^{+} \boldsymbol{e}^{-}+\boldsymbol{E}
$$

For decay topology $Z \rightarrow \ell^{+} \ell^{-}+\not ̈$, we consider SM background $j \ell^{+} \ell^{-}$and irreducible $j \ell^{-} \ell^{+} \nu \bar{\nu}$ with the same reason. The basic cuts are one jet with $p_{T}^{j}>60 \mathrm{GeV}$, missing energy $E_{T}>50 \mathrm{GeV}$, and two leptons with $p_{T}^{\ell}>20 \mathrm{GeV}$. After checking the kinematic variable distribution, we propose further cuts to optimize our signal,

$$
p_{T}^{j}>90 \mathrm{GeV}, \quad p_{T}^{\ell_{1}}<80 \mathrm{GeV} .
$$

For topologies $3 \mathrm{~A}$ and $3 \mathrm{~B}$, we have added the same additional $5 \mathrm{GeV}$ window cut on the invariant mass of the dilepton. The corresponding efficiency has been listed in parentheses in the $\epsilon$ column in Table II. For the HL-LHC $\left(3 \mathrm{ab}^{-1}\right)$, the future sensitivity reaches for exotic $Z$-decay topologies $3 \mathrm{~A}, 3 \mathrm{~B}, 3 \mathrm{D}$, and $3 \mathrm{~F}$ are $(3-11) \times 10^{-6}$, $(3 \sim 12) \times 10^{-6}, 2.0 \times 10^{-5}$, and $1.6 \times 10^{-5}$, respectively, and are plotted in Fig. 16. The sensitivity range for the topology 3A and 3B is indicated by the light brown shaded region in Fig. 16.

$$
\text { 4. } Z \rightarrow \ddot{j}+\boldsymbol{E}^{\prime}
$$

For decay topology $Z \rightarrow j j+\not{E}$, we generate signal events $\gamma Z$ to suppress the QCD background and consider the SM background $\gamma j$ and irreducible $\gamma j j \nu \bar{\nu}$. The basic cuts are two jets with $p_{T}^{j}>30 \mathrm{GeV}$, missing energy $E_{T}>50 \mathrm{GeV}$, and one photon with $p_{T}^{\gamma}>60 \mathrm{GeV}$. After checking the kinematic variable distribution, we propose further cuts to optimize our signal:

$p_{T}^{j_{1}}<100 \mathrm{GeV}, \quad E_{T}^{\dagger}>60 \mathrm{GeV}, \quad p_{T}^{\gamma}>90 \mathrm{GeV}$.

For the HL-LHC $\left(3 \mathrm{ab}^{-1}\right)$, the future sensitivity reaches for exotic $Z$-decay topologies $4 \mathrm{~A}, 4 \mathrm{~B}$, and $4 \mathrm{C}$ are 0.0136 , $3.45 \times 10^{-3}$, and $5.07 \times 10^{-3}$, respectively, and are plotted in Fig. 16.

$$
\text { 5. } \boldsymbol{Z} \rightarrow(\boldsymbol{J J})(\boldsymbol{J} \boldsymbol{J})
$$

For decay topology $Z \rightarrow(J J)(J J)$ which is fully hadronic, we generate signal events $\gamma Z$ to suppress the QCD background and consider the SM background $\gamma J$ matched with $\gamma J J$ by PYTHIA and irreducible $\gamma J \nu \bar{\nu}$ matched with $\gamma J J \nu \bar{\nu}$, where $J$ can be light flavor jets $j$ or a $b$-tagged jet $b$. We require at least four jets with $p_{T}^{J}>60 \mathrm{GeV}$ and one photon with $p_{T}^{\gamma}>60 \mathrm{GeV}$. We propose further cuts to optimize our signal,

$$
p_{T}^{J_{1}}>120 \mathrm{GeV}, \quad m_{J J J J}<250 \mathrm{GeV},
$$

and the cut efficiencies for the signal and SM background are given in Table II. Note we have generated the SM 
backgrounds with the light flavor jet and $b$ jet separately. Both of them can contribute to the background of the corresponding signal topologies $5 \mathrm{~A}, 5 \mathrm{~B}$, and $5 \mathrm{C}$ with $b$-tagging efficiency reweighting. For the HL-LHC $\left(3 \mathrm{ab}^{-1}\right)$, the sensitivity reaches for exotic Z-decay topologies 5A, 5B, and 5C are 0.0126, 0.0172, and 0.00915, respectively, and are plotted in Fig. 16. It is not surprising that the sensitivity for fully hadronic decay of $Z$ at the HL-LHC cannot compete with future $e^{+} e^{-}$collider because of the huge QCD background.

Using the jet substructure technique can probably achieve better sensitivities in the exotic hadronic $Z$-decay topologies. CMS at the $13 \mathrm{TeV}$ LHC has searched for light vector resonance which decays into quark pair in association with a high $p_{T}$ jet to make the light vector gauge boson highly boosted [136], the decay products of which are merged into a single jet. The characteristic feature of the signal is a single massive jet with a two-prong substructure produced in association with a jet from initial state radiation. The SM process $j Z \rightarrow j(j j)$ has been nicely reconstructed. In the exotic decay topology $Z \rightarrow(J J)(J J)$, one would look for the four-prong substructure in the fat jet to suppress the SM $j Z$ background. For the final state including $b$ jets, $b$-tagging techniques in the jet substructure could further help to reduce the SM QCD background, which already helps with observing a local significance of 5.1 standard deviations for the first time in the single jet topology in the $Z \rightarrow b b$ process [137].

\section{6. $Z \rightarrow \gamma \gamma \gamma$}

The last exotic $Z$-decay search is $Z \rightarrow \gamma \gamma \gamma$, which has been performed by ATLAS at the $8 \mathrm{TeV}$ LHC [122] with $\mathcal{L}=20 \mathrm{fb}^{-1}$. The corresponding constraint is $\operatorname{BR}(Z \rightarrow \gamma \gamma \gamma)<2.2 \times 10^{-6}$. It is hard for us to reliably study this topology due to the difficulty in simulating the fake photons from QCD backgrounds. Instead, we do a simple rescaling according to the HL-LHC integrated luminosity $3 \mathrm{ab}^{-1}$, which gives the limit $\mathrm{BR}(Z \rightarrow \gamma \gamma \gamma)<1.8 \times 10^{-7}$.

\section{Summary}

In Fig. 16, we see the sensitivity of the exotic Z-decay branching ratios at the HL-LHC generally cannot compete with the future $Z$ factory because of the large QCD background. The $Z$ exotic decay products are typically rather soft. Requiring another hard radiation can help with triggering and making the Z-decay products more energetic. At the same time, it will reduce the signal rate significantly. For exotic $Z$ decay with missing energy in the final state, another important background can come from the mismeasurement of the QCD jets. Since the missing energy from $Z$ decay tends to be small, this background can be significant. For photons in final states, there can be fake photons from QCD, which we have not considered. For hadronic exotic $Z$ decay, the situation at the HL-LHC is even worse.

\section{CONCLUSION AND DISCUSSION}

We have presented a comprehensive study on exotic $Z$ decay at future $Z$ factories, with emphasis on its prospects for exploring dark sector models. There are many dark sector models that can give rise to exotic Z-decay modes, many of which contain missing energy in the final states. A $Z$ factory provides a clean environment for decay modes which can be overwhelmed by a large background at hadron colliders. Another advantage of searching for such exotic $Z$ decay at future $e^{+} e^{-}$colliders is the ability to reconstruct the full missing 4-momentum, while we can only reconstruct the missing transverse momentum at hadron colliders. We have demonstrated the capability of exotic $Z$ decay at a future $Z$ factory to provide the leading constraint in comparison with existing collider limits, future HL-LHC projections, and current DM searches.

We classify final states of the exotic decays with the number of resonances and possible topologies it could have. We make projections on the sensitivity on the branching ratio of exotic $Z$ decay at a future $Z$ factory. For final states with missing energy, it can provide limits on the BR down to $10^{-6}-10^{-8.5}$ for Giga $Z$ and $10^{-7.5}-10^{-11}$ for Tera $Z$. The sensitivities on the BR for different final states are roughly ordered from high to low as $E^{+} \ell^{+} \ell^{-} \sim E_{\gamma \gamma} \gamma E^{\prime} J J$, and $E_{\gamma}$, due to the size of the SM backgrounds for each mode. In the same final states, it is quite clear the SM backgrounds for signal with more resonances can be better suppressed. In addition to the final states with missing energy, we also selectively studied the fully visible final states $(J J)(J J)$ and $(\gamma \gamma) \gamma$, where the first one contains two resonances and the second one contains one resonance. It is interesting to look for purely hadronic final states at future $Z$ factories because it has much less QCD background in comparison with the hadron collider. We found it can provide limits on the BR down to $10^{-5}-10^{-6.5}$ for Giga $Z$ and $10^{-6.5}-10^{-8}$ for Tera $Z$. The sensitivity to the final states with a $b$ jet is better than those with light flavor jets due to smaller SM backgrounds.

We have also made estimates of the reach of the HL-LHC on the exotic $Z$-decay modes. The decay products tend to be soft and difficult for the LHC searches. There are also large QCD backgrounds. We considered the cases with additional energetic initial state radiation, which can help suppress these backgrounds. However, this also reduced the signal rate. Therefore, for the channels we considered here, it is very hard for the HL-LHC to compete with the future $Z$ factory. The one exception is the $(\gamma \gamma) \gamma$ channel. The sensitivity on the BR can reach $10^{-7}$ for Giga $Z$ and a few $10^{-9}$ for Tera $Z$. The corresponding HL-LHC sensitivity is rescaled from an exiting study at $8 \mathrm{TeV}$ by rescaling, and it can be comparable to that of the $Z$ factory. 
We have studied four representative models in Sec. III, namely the Higgs portal with scalar DM, vector-portal DM model, MIDM and RayDM, and axionlike particle model. In the Higgs portal model with DM, the decay topology $Z \rightarrow \tilde{s} Z^{*} \rightarrow(\bar{\chi} \chi)+\ell^{+} \ell^{-}$has been studied. Future $Z$ factories can provide the leading constraint on mixing angle $\sin \alpha$ between the SM Higgs and dark singlet scalar mediator. The constraint from $Z \rightarrow \tilde{s} \gamma$ via the loop effect has also been considered but is weaker due to loop suppression and a larger SM background. In the vectorportal DM model, the decay topologies $\tilde{Z} \rightarrow \tilde{A}^{\prime} S S^{*} \rightarrow$ $\left(\ell^{+} \ell^{-}\right) E^{\prime}$ and $\tilde{Z} \rightarrow \tilde{A}^{\prime} \tilde{\phi} \rightarrow \ell^{+} \ell^{-}\left(\not E^{\prime}\right)$ are studied. The first one simply arises when DM is a scalar and charged under $U(1)_{D}$, and the second one is a dark Higgs bremsstrahlung process. We found that the limits from the exotic $Z$ decay provide competitive and complementary constraints with DM direct detection, while the other collider limits are much weaker. In the MIDM and RayDM models, the decay topologies $Z \rightarrow \chi_{2} \chi_{1} \rightarrow\left(\chi_{1} \gamma\right) \chi_{1}$ from the MIDM operator and $Z \rightarrow \chi_{1} \chi_{1} \gamma$ from the RayDM operator has been considered. Both operators can originate from heavy fermions and scalars in the loop, which couples to DM. The constraint on the MIDM operator is much stronger than the constraint on RayDM. It is also much better than the gamma-line search in indirect detection and future hadron collider projections. In the axionlike particles model, the decay topologies $Z \rightarrow a \gamma \rightarrow(\gamma \gamma) \gamma$ and $Z \rightarrow a \gamma \rightarrow(\mathscr{E}) \gamma$ have been considered, in which, in the first one, the axionlike particles decay promptly into two photons and, in the second one, they decay outside the detector. We find the future $Z$ factory can provide the leading constraint on $\Lambda_{\mathrm{aBB}}$ compared to limits from the LEP and LHC.

All in all, the exotic $Z$-decay searches can provide unique tests on dark sector models at the future $Z$ factory, especially when missing energy and/or hadronic objects appear in the final states. We explicitly analyze four representative dark sector models and find the exotic $Z$-decay searches can provide the leading and complementary limits to the current and future collider searches and DM searches. It can also cover parameter spaces of DM models with the relic abundance requirement, which provides a complementary cross-check on the DM problem.

\section{ACKNOWLEDGMENTS}

We would like to thank Oliver Fischer, Manqi Ruan, and Felix Yu for useful discussions and comments. J. L. is supported by the Oehme Fellowship. L. T. W. is supported by the DOE Grant No. DE-SC-0013642. X. P. W. is supported by the U.S. Department of Energy under Contract No. DE-AC02-06CH11357. W. X. is supported by the U.S. Department of Energy under Grants No. DESC-00012567, No. DE-SC-00015476, and No. 669668NEO-NAT-ERC-AdG-2014. The work of J. L. and X. P. W. was partially supported by the Cluster of Excellence Precision Physics, Fundamental Interactions and Structure of Matter (PRISMA-EXC 1098), the German Research Foundation (DFG) under Grants No. KO 4820/11 and No. FOR 2239 and by the European Research Council (ERC) under the European Unions Horizon 2020 research and innovation program (Grant No. 637506, "vDirections").

\section{APPENDIX: ANNIHILATION CROSS SECTION FOR SCALAR DM WITH VECTOR PORTAL}

We calculate the annihilation cross sections of scalar DM into SM fermions. The scalar DM is charged under $U(1)_{D}$ as in Eq. (17), and the kinetic mixing induced interactions with the SM sector are given in Eq. (16), which includes both s-channel $\tilde{A}^{\prime}$ and $\tilde{Z}$ mediation. The annihilation cross sections for one generation are given,

$$
\begin{aligned}
\sigma v_{S S \rightarrow u \bar{u}}= & \frac{g_{D}^{2} e^{2} \epsilon^{2} \sqrt{s-4 m_{u}^{2}}\left(s-4 m_{S}^{2}\right)}{576 \pi s^{3 / 2} \cos \theta_{w}^{4}\left(m_{\tilde{K}}^{2}-m_{Z}^{2}\right)^{2}\left(\left(s-m_{\tilde{K}}^{2}\right)^{2}+m_{\tilde{K}}^{2} \Gamma_{\tilde{K}}^{2}\right)\left(\left(s^{2}-m_{\tilde{Z}}^{2}\right)^{2}+m_{\tilde{Z}}^{2} \Gamma_{\tilde{Z}}^{2}\right)} \\
& \times\left[\left(17 s+7 m_{u}^{2}\right)\left(s^{2}\left(m_{\tilde{K}}^{2}-m_{\tilde{Z}}^{2}\right)^{2}+m_{\tilde{K}}^{2} m_{\tilde{Z}}^{2}\left(m_{\tilde{Z}} \Gamma_{\tilde{K}}-m_{\tilde{K}} \Gamma_{\tilde{Z}}\right)^{2}\right)\right. \\
& -40 \cos \theta_{w}^{2} m_{\tilde{Z}}^{2}\left(s+2 m_{u}^{2}\right)\left(s\left(m_{\tilde{K}}^{2}-m_{\tilde{Z}}^{2}\right)^{2}+m_{\tilde{K}} m_{\tilde{Z}}\left(m_{\tilde{Z}} \Gamma_{\tilde{K}}-m_{\tilde{K}} \Gamma_{\tilde{Z}}\right)\left(m_{\tilde{K}} \Gamma_{\tilde{K}}-m_{\tilde{Z}} \Gamma_{\tilde{Z}}\right)\right) \\
& \left.+32 \cos \theta_{w}^{4} m_{\tilde{Z}}^{4}\left(s+2 m_{u}^{2}\right)\left(m_{\tilde{K}}^{4}+m_{\tilde{K}}^{2}\left(\Gamma_{\tilde{K}}^{2}-2 m_{\tilde{Z}}^{2}\right)-2 m_{\tilde{K}} m_{\tilde{Z}} \Gamma_{\tilde{K}} \Gamma_{\tilde{Z}}+m_{\tilde{Z}}^{2}\left(m_{\tilde{Z}}^{2}+\Gamma_{\tilde{Z}}^{2}\right)\right)\right], \\
\sigma v_{S S \rightarrow d \bar{d}}= & \frac{g_{D}^{2} e^{2} \epsilon^{2} \sqrt{s-4 m_{d}^{2}}\left(s-4 m_{S}^{2}\right)}{} \\
& \times\left[\left(5 s-17 m_{d}^{2}\right)\left(s^{2}\left(m_{\tilde{K}}^{2}-m_{\tilde{Z}}^{2}\right)^{2}+m_{\tilde{K}}^{2} m_{\tilde{Z}}^{2}\left(m_{\tilde{Z}} \Gamma_{\tilde{K}}-m_{\tilde{K}} \Gamma_{\tilde{Z}}\right)^{2}\right)\right. \\
& -4 \cos \theta_{w}^{2} m_{\tilde{Z}}^{2}\left(s+2 m_{d}^{2}\right)\left(s\left(m_{\tilde{K}}^{2}-m_{\tilde{Z}}^{2}\right)^{2}+m_{\tilde{K}} m_{\tilde{Z}}\left(m_{\tilde{Z}} \Gamma_{\tilde{K}}-m_{\tilde{K}} \Gamma_{\tilde{Z}}\right)\left(m_{\tilde{K}} \Gamma_{\tilde{K}}-m_{\tilde{Z}} \Gamma_{\tilde{Z}}\right)\right) \\
& \left.+8 \cos \theta_{w}^{4} m_{\tilde{Z}}^{4}\left(s+2 m_{d}^{2}\right)\left(m_{\tilde{K}}^{4}+m_{\tilde{K}}^{2}\left(-2 m_{\tilde{Z}}^{2}+\Gamma_{\tilde{K}}^{2}\right)-2 m_{\tilde{K}} m_{\tilde{Z}} \Gamma_{\tilde{K}} \Gamma_{\tilde{Z}}+m_{\tilde{Z}}^{2}\left(m_{\tilde{Z}}^{2}+\Gamma_{\tilde{Z}}^{2}\right)\right)\right],
\end{aligned}
$$




$$
\begin{aligned}
\sigma v_{S S \rightarrow \bar{\nu}}= & \frac{g_{D}^{2} e^{2} \epsilon^{2}\left(s-4 m_{S}^{2}\right)\left(s^{2}\left(m_{\tilde{K}}^{2}-m_{\tilde{Z}}^{2}\right)^{2}+m_{\tilde{K}}^{2} m_{\tilde{Z}}^{2}\left(m_{\tilde{Z}} \Gamma_{\tilde{K}}-m_{\tilde{K}} \Gamma_{\tilde{Z}}\right)^{2}\right)}{192 \cos \theta_{w}^{4} \pi\left(m_{\tilde{K}}^{2}-m_{\tilde{Z}}^{2}\right)^{2}\left(\left(s-m_{\tilde{K}}^{2}\right)^{2}+m_{\tilde{K}}^{2} \Gamma_{\tilde{K}}^{2}\right)\left(\left(s-m_{\tilde{Z}}^{2}\right)^{2}+m_{\tilde{Z}}^{2} \Gamma_{\tilde{Z}}^{2}\right)}, \\
\sigma v_{S S \rightarrow \bar{l}}= & \frac{g_{D}^{2} e^{2} \epsilon^{2} \sqrt{s-4 m_{l}^{2}}\left(s-4 m_{S}^{2}\right)}{192 \pi \cos \theta_{w}^{4} s^{3 / 2}\left(m_{\tilde{K}}^{2}-m_{\tilde{Z}}^{2}\right)^{2}\left(\left(s-m_{\tilde{K}}^{2}\right)^{2}+m_{\tilde{K}}^{2} \Gamma_{\tilde{K}}^{2}\right)\left(\left(s-m_{\tilde{Z}}^{2}\right)^{2}+m_{\tilde{Z}}^{2} \Gamma_{\tilde{Z}}^{2}\right)} \\
& \times\left[\left(5 s+7 m_{l}^{2}\right)\left(s^{2}\left(m_{\tilde{K}}^{2}-m_{\tilde{Z}}^{2}\right)^{2}+m_{\tilde{K}}^{2} m_{\tilde{Z}}^{2}\left(m_{\tilde{Z}} \Gamma_{\tilde{K}}-m_{\tilde{K}} \Gamma_{\tilde{Z}}\right)^{2}\right)\right. \\
& -12 \cos \theta_{w}^{2} m_{\tilde{Z}}^{2}\left(s+2 m_{l}^{2}\right)\left(s\left(m_{\tilde{K}}^{2}-m_{\tilde{Z}}^{2}\right)^{2}+m_{\tilde{K}} m_{\tilde{Z}}\left(m_{\tilde{Z}} \Gamma_{\tilde{K}}-m_{\tilde{K}} \Gamma_{\tilde{Z}}\right)\left(m_{\tilde{K}} \Gamma_{\tilde{K}}-m_{\tilde{Z}} \Gamma_{\tilde{Z}}\right)\right) \\
& \left.+8 \cos \theta_{w}^{4} m_{\tilde{Z}}^{4}\left(s+2 m_{l}^{2}\right)\left(m_{\tilde{K}}^{4}+m_{\tilde{K}}^{2}\left(-2 m_{\tilde{Z}}^{2}+\Gamma_{\tilde{K}}^{2}\right)-2 m_{\tilde{K}} m_{\tilde{Z}} \Gamma_{\tilde{K}} \Gamma_{\tilde{Z}}+m_{\tilde{Z}}^{2}\left(m_{\tilde{Z}}^{2}+\Gamma_{\tilde{Z}}^{2}\right)\right)\right],
\end{aligned}
$$

where we see the cross sections are p-wave suppressed.

[1] M. Bicer et al. (TLEP Design Study Working Group Collaboration), First look at the physics case of TLEP, J. High Energy Phys. 01 (2014) 164.

[2] D. d'Enterria, Physics case of FCC-ee, Frascati Phys. Ser. 61, 17 (2016).

[3] D. d'Enterria, Particle Physics at the Year of Light, in Proceedings of the 17th Lomonosov Conference on Elementary Particle Physics (World Scientific, Singapore, 2017), p. $182-191$.

[4] CEPC-SPPC Study Group, CEPC-SPPC Preliminary Conceptual Design Report. 1. Physics and Detector, Reports No. IHEP-CEPC-DR-2015-01, IHEP-TH-2015-01, IHEPEP-2015-01.

[5] S. Schael et al. (SLD Electroweak Group, DELPHI, ALEPH, SLD, SLD Heavy Flavour Group, OPAL, LEP Electroweak Working Group, and L3 Collaborations), Precision electroweak measurements on the $Z$ resonance, Phys. Rep. 427, 257 (2006).

[6] R. Essig et al., in Proceedings of the 2013 Community Summer Study on the Future of U.S. Particle Physics: Snowmass on the Mississippi (CSS2013), Minneapolis, MN, 2013 (unpublished).

[7] J. Alexander et al., Dark Sectors 2016 Workshop: Community report (2016), arXiv:1608.08632.

[8] M. Battaglieri et al., US Cosmic Visions: New Ideas in Dark Matter 2017: Community report, arXiv:1707.04591.

[9] V. Silveira and A. Zee, Scalar phantoms, Phys. Lett. 161B, 136 (1985).

[10] J. McDonald, Gauge singlet scalars as cold dark matter, Phys. Rev. D 50, 3637 (1994).

[11] C. P. Burgess, M. Pospelov, and T. ter Veldhuis, The minimal model of nonbaryonic dark matter: A singlet scalar, Nucl. Phys. B619, 709 (2001).

[12] B. Patt and F. Wilczek, Higgs-field portal into hidden sectors, arXiv:hep-ph/0605188.

[13] Y. G. Kim and K. Y. Lee, The minimal model of fermionic dark matter, Phys. Rev. D 75, 115012 (2007).

[14] V. Barger, P. Langacker, M. McCaskey, M. J. RamseyMusolf, and G. Shaughnessy, LHC phenomenology of an extended standard model with a real scalar singlet, Phys. Rev. D 77, 035005 (2008).
[15] Y. G. Kim, K. Y. Lee, and S. Shin, Singlet fermionic dark matter, J. High Energy Phys. 05 (2008) 100.

[16] J. M. Cline, K. Kainulainen, P. Scott, and C. Weniger, Update on scalar singlet dark matter, Phys. Rev. D 88, 055025 (2013); Erratum, 92, 039906 (2015).

[17] L. B. Okun, Limits of electrodynamics: Paraphotons?, Zh. Eksp. Teor. Fiz. 83, 892 (1982) [Sov. Phys. JETP 56, 502 (1982)].

[18] B. Holdom, Two U(1)'s and epsilon charge shifts, Phys. Lett. 166B, 196 (1986).

[19] M. Pospelov, A. Ritz, and M. B. Voloshin, Secluded WIMP dark matter, Phys. Lett. B 662, 53 (2008).

[20] N. Arkani-Hamed and N. Weiner, LHC signals for a superunified theory of dark matter, J. High Energy Phys. 12 (2008) 104.

[21] N. Arkani-Hamed, D. P. Finkbeiner, T. R. Slatyer, and N. Weiner, A theory of dark matter, Phys. Rev. D 79, 015014 (2009).

[22] M. Pospelov, Secluded U(1) below the weak scale, Phys. Rev. D 80, 095002 (2009).

[23] R. D. Peccei and H. R. Quinn, $C P$ Conservation in the Presence of Instantons, Phys. Rev. Lett. 38, 1440 (1977).

[24] S. Weinberg, A New Light Boson?, Phys. Rev. Lett. 40, 223 (1978).

[25] F. Wilczek, Problem of Strong p and t Invariance in the Presence of Instantons, Phys. Rev. Lett. 40, 279 (1978).

[26] J. M. Frere, D. R. T. Jones, and S. Raby, Fermion masses and induction of the weak scale by supergravity, Nucl. Phys. B222, 11 (1983).

[27] A. E. Nelson and N. Seiberg, R symmetry breaking versus supersymmetry breaking, Nucl. Phys. B416, 46 (1994).

[28] J. Bagger, E. Poppitz, and L. Randall, The R axion from dynamical supersymmetry breaking, Nucl. Phys. B426, 3 (1994).

[29] J. P. Conlon, The QCD axion and moduli stabilisation, J. High Energy Phys. 05 (2006) 078.

[30] P. Svrcek and E. Witten, Axions in string theory, J. High Energy Phys. 06 (2006) 051. 
[31] Y. Nomura and J. Thaler, Dark matter through the axion portal, Phys. Rev. D 79, 075008 (2009).

[32] A. Arvanitaki, S. Dimopoulos, S. Dubovsky, N. Kaloper, and J. March-Russell, String axiverse, Phys. Rev. D 81, 123530 (2010).

[33] J. Jaeckel and A. Ringwald, The low-energy frontier of particle physics, Annu. Rev. Nucl. Part. Sci. 60, 405 (2010).

[34] B. S. Acharya, K. Bobkov, and P. Kumar, An M theory solution to the strong $C P$ problem and constraints on the axiverse, J. High Energy Phys. 11 (2010) 105.

[35] A. Ringwald, Exploring the role of axions and other WISPs in the dark universe, Phys. Dark Universe 1, 116 (2012).

[36] K. Sigurdson, M. Doran, A. Kurylov, R. R. Caldwell, and M. Kamionkowski, Dark-matter electric and magnetic dipole moments, Phys. Rev. D 70, 083501 (2004); Erratum, 73, 089903 (2006).

[37] E. Masso, S. Mohanty, and S. Rao, Dipolar Dark Matter, Phys. Rev. D 80, 036009 (2009).

[38] S. Chang, N. Weiner, and I. Yavin, Magnetic inelastic dark matter, Phys. Rev. D 82, 125011 (2010).

[39] N. Weiner and I. Yavin, How dark are Majorana WIMPs? Signals from MiDM and Rayleigh dark matter, Phys. Rev. D 86, 075021 (2012).

[40] N. Weiner and I. Yavin, UV completions of magnetic inelastic and Rayleigh dark matter for the Fermi Line(s), Phys. Rev. D 87, 023523 (2013).

[41] A. Hook, E. Izaguirre, and J. G. Wacker, Model independent bounds on kinetic mixing, Adv. High Energy Phys. 2011, 859762 (2011).

[42] D. Curtin, R. Essig, S. Gori, and J. Shelton, Illuminating dark photons with high-energy colliders, J. High Energy Phys. 02 (2015) 157.

[43] A. Blondel, E. Graverini, N. Serra, M. Shaposhnikov (FCC-ee study Team Collaboration), Search for heavy right handed neutrinos at the FCC-ee, Nucl. Part. Phys. Proc. 273-275, 1883 (2016).

[44] A. Abada, V. De Romeri, S. Monteil, J. Orloff, and A. M. Teixeira, Indirect searches for sterile neutrinos at a highluminosity Z-factory, J. High Energy Phys. 04 (2015) 051.

[45] M. Carena, A. de Gouvea, A. Freitas, and M. Schmitt, Invisible $\mathrm{Z}$ boson decays at $e^{+} e^{-}$colliders, Phys. Rev. D 68, 113007 (2003).

[46] H.-W. Ke and X.-Q. Li, Search for $B_{c}(n s)$ via the $B_{c}(n s) \rightarrow B_{c}(m s) \pi^{+} \pi^{-}$transition at LHCb and $Z^{0}$ factory, Sci. China Phys. Mech. Astron. 53, 2019 (2010).

[47] Y. Jin, Z. Si, Q. Xie, and T. Yao, An investigation of hadronization mechanism at $Z^{0}$ factory, Sci. China Phys. Mech. Astron. 53, 1998 (2010).

[48] T.-C. Huang and F. Petriello, Rare exclusive decays of the Z-boson revisited, Phys. Rev. D 92, 014007 (2015).

[49] Q.-J. Xu and C.-H. Chang, Suggestion for measuring the weak dipole moment of $\tau$ lepton at Z factory, Phys. Rev. D 91, 013011 (2015).

[50] G. Durieux, Y. Grossman, M. König, E. Kuflik, and S. Ray, Rare $\mathrm{Z}$ decays and neutrino flavor universality, Phys. Rev. D 93, 093005 (2016).

[51] J. Cao, Z. Heng, and J. M. Yang, Rare Z-decay into light $C P$-odd Higgs bosons: A comparative study in different new physics models, J. High Energy Phys. 11 (2010) 110.
[52] L. Wang and X.-F. Han, Rare Z-decay into light pseudoscalar bosons in the simplest little Higgs model, Nucl. Phys. B853, 625 (2011).

[53] F. Domingo and T. Lenz, W mass and Leptonic Z-decays in the NMSSM, J. High Energy Phys. 07 (2011) 101.

[54] L. Wang and X.-F. Han, Light Higgs decay modes of Z-boson in the left-right twin Higgs model, Nucl. Phys. B850, 233 (2011).

[55] P. Ghosh, D. E. Lpez-Fogliani, V. A. Mitsou, C. Muoz, and R. Ruiz de Austri, Hunting physics beyond the standard model with unusual $W^{ \pm}$and $Z$ decays, Phys. Rev. D 91, 035020 (2015).

[56] T. Flacke, C. Frugiuele, E. Fuchs, R. S. Gupta, and G. Perez, Phenomenology of relaxion-Higgs mixing, J. High Energy Phys. 06 (2017) 050.

[57] N. Blinov, E. Izaguirre, and B. Shuve, Rare $Z$ boson decays to a hidden sector, Phys. Rev. D 97, 015009 (2018).

[58] Y. Gao and M. Jin, Z-pole test of effective dark matter diboson interactions at the CEPC, arXiv:1712.02140.

[59] Z.-H. Yu, X.-J. Bi, Q.-S. Yan, and P.-F. Yin, Dark matter searches in the mono- $Z$ channel at high energy $e^{+} e^{-}$ colliders, Phys. Rev. D 90, 055010 (2014).

[60] M. Fabbrichesi, E. Gabrielli, and B. Mele, $Z$ boson decay into light and darkness, arXiv:1712.05412.

[61] M. Acciarri et al. (L3 Collaboration), Search for neutral Higgs boson production through the process $e^{+} e^{-} \rightarrow$ $Z^{*} H^{0}$, Phys. Lett. B 385, 454 (1996).

[62] X. Guo, T. Feng, S. Zhao, H.-W. Ke, and X.-Q. Li, Constraining parameter space of the little Higgs model using data from tera-Z factory and ILC, Chin. Phys. C 39, 023103 (2015).

[63] S. Schael et al. (DELPHI, OPAL, ALEPH, LEP Working Group for Higgs Boson Searches, and L3 Collaborations), Search for neutral MSSM Higgs bosons at LEP, Eur. Phys. J. C 47, 547 (2006).

[64] M. Acciarri et al. (L3 Collaboration), Search for new physics in energetic single photon production in $e^{+} e^{-}$ annihilation at the $Z$ resonance, Phys. Lett. B 412, 201 (1997).

[65] R. Akers et al. (OPAL Collaboration), Measurement of single photon production in $e^{+} e^{-}$collisions near the $Z^{0}$ resonance, Z. Phys. C 65, 47 (1995).

[66] M. Acciarri et al. (L3 Collaboration), Single and multiphoton events with missing energy in $e^{+} e^{-}$collisions at $\sqrt{S}$-189-GeV, Phys. Lett. B 470, 268 (1999).

[67] M. Acciarri et al. (L3 Collaboration), Search for anomalous $Z \rightarrow \gamma \gamma \gamma$ events at LEP, Phys. Lett. B345, 609 (1995).

[68] P. J. Fox, R. Harnik, J. Kopp, and Y. Tsai, LEP shines light on dark matter, Phys. Rev. D 84, 014028 (2011).

[69] E. Aprile et al. (XENON Collaboration), First Dark Matter Search Results from the XENON1T Experiment, Phys. Rev. Lett. 119, 181301 (2017).

[70] D. S. Akerib et al. (LUX Collaboration), Results from a Search for Dark Matter in the Complete LUX Exposure, Phys. Rev. Lett. 118, 021303 (2017).

[71] A. Tan et al. (PandaX-II Collaboration), Dark Matter Results from First 98.7 Days of Data from the PandaX-II Experiment, Phys. Rev. Lett. 117, 121303 (2016). 
[72] G. Angloher et al. (CRESST Collaboration), Results on light dark matter particles with a low-threshold CRESST-II detector, Eur. Phys. J. C 76, 25 (2016).

[73] R. Agnese et al. (SuperCDMS Collaboration), New Results from the Search for Low-Mass Weakly Interacting Massive Particles with the CDMS Low Ionization Threshold Experiment, Phys. Rev. Lett. 116, 071301 (2016).

[74] M. Ackermann et al. (Fermi-LAT Collaboration), Search for gamma-ray spectral lines with the Fermi Large Area Telescope and dark matter implications, Phys. Rev. D 88, 082002 (2013).

[75] M. Ackermann et al. (Fermi-LAT Collaboration), Searching for Dark Matter Annihilation from Milky Way Dwarf Spheroidal Galaxies with Six Years of Fermi Large Area Telescope Data, Phys. Rev. Lett. 115, 231301 (2015).

[76] M. Aguilar et al. (AMS Collaboration), Electron and Positron Fluxes in Primary Cosmic Rays Measured with the Alpha Magnetic Spectrometer on the International Space Station, Phys. Rev. Lett. 113, 121102 (2014).

[77] P. A. R. Ade et al. (Planck Collaboration), Planck 2015 results. XIII. Cosmological parameters, Astron. Astrophys. 594, A13 (2016).

[78] S. Baek, P. Ko, and W.-I. Park, Search for the Higgs portal to a singlet fermionic dark matter at the LHC, J. High Energy Phys. 02 (2012) 047.

[79] S. Baek, P. Ko, W.-I. Park, and E. Senaha, Vacuum structure and stability of a singlet fermion dark matter model with a singlet scalar messenger, J. High Energy Phys. 11 (2012) 116.

[80] M. Fairbairn and R. Hogan, Singlet fermionic dark matter and the electroweak phase transition, J. High Energy Phys. 09 (2013) 022.

[81] S. Esch, M. Klasen, and C. E. Yaguna, Detection prospects of singlet fermionic dark matter, Phys. Rev. D 88, 075017 (2013).

[82] S. Baek, P. Ko, and W.-I. Park, Invisible Higgs decay width vs. dark matter direct detection cross section in Higgs portal dark matter models, Phys. Rev. D 90, 055014 (2014).

[83] Z. Bagherian, M. M. Ettefaghi, Z. Haghgouyan, and R. Moazzemi, A new parameter space study of the fermionic cold dark matter model, J. Cosmol. Astropart. Phys. 10 (2014) 033.

[84] A. Freitas, S. Westhoff, and J. Zupan, Integrating in the Higgs portal to fermion dark matter, J. High Energy Phys. 09 (2015) 015.

[85] J. Kumar and D. Marfatia, Matrix element analyses of dark matter scattering and annihilation, Phys. Rev. D 88, 014035 (2013).

[86] K. Kainulainen, K. Tuominen, and V. Vaskonen, Selfinteracting dark matter and cosmology of a light scalar mediator, Phys. Rev. D 93, 015016 (2016); Erratum, 95, 079901 (2017).

[87] G. Aad et al. (ATLAS Collaboration), Constraints on new phenomena via Higgs boson couplings and invisible decays with the ATLAS detector, J. High Energy Phys. 11 (2015) 206.

[88] V. Khachatryan et al. (CMS Collaboration), Searches for invisible decays of the Higgs boson in pp collisions at $\sqrt{s}=7,8$, and 13 TeV, J. High Energy Phys. 02 (2017) 135.
[89] The ATLAS Collaboration, Projections for measurements of Higgs boson cross sections, branching ratios and coupling parameters with the ATLAS detector at a HLLHC, Technical Report ATL-PHYS-PUB-2013-014, 2013.

[90] CMS Collaboration, in Proceedings of the 2013 Community Summer Study on the Future of U.S. Particle Physics: Snowmass on the Mississippi (CSS2013), Minneapolis, MN, 2013 (unpublished).

[91] K. Fujii et al., Physics Case for the $250 \mathrm{GeV}$ Stage of the International Linear Collider, arXiv:1710.07621.

[92] G. Aad et al. (ATLAS and CMS Collaborations), Measurements of the Higgs boson production and decay rates and constraints on its couplings from a combined ATLAS and CMS analysis of the LHC pp collision data at $\sqrt{s}=7$ and 8 TeV, J. High Energy Phys. 08 (2016) 045.

[93] S. Dawson et al., in Proceedings of the 2013 Community Summer Study on the Future of U.S. Particle Physics: Snowmass on the Mississippi (CSS2013), Minneapolis, MN, 2013 (unpublished).

[94] M. Acciarri et al. (L3 Collaboration), Missing mass spectra in hadronic events from $e^{+} e^{-}$collisions at $s^{* *}(1 / 2)=$ $161-\mathrm{GeV}-172-\mathrm{GeV}$ and limits on invisible Higgs decay, Phys. Lett. B 418, 389 (1998).

[95] P. Abreu et al. (DELPHI Collaboration), A search for invisible Higgs bosons produced in $e^{+} e^{-}$interactions at LEP-2 energies, Phys. Lett. B 459, 367 (1999).

[96] OPAL, DELPHI, LEP Higgs Working for Higgs boson searches, L3 CERN, and ALEPH Collaborations, in Proceedings of the 20th International Symposium, LP 2001, Rome, 2001 (unpublished).

[97] J. Liu, X.-P. Wang, and F. Yu, A tale of two portals: Testing light, hidden new physics at future $e^{+} e^{-}$colliders, J. High Energy Phys. 06 (2017) 077.

[98] M. Ruan, Higgs measurement at $e^{+} e^{-}$circular colliders, Nucl. Part. Phys. Proc. 273-275, 857 (2016).

[99] S. Cassel, D. M. Ghilencea, and G. G. Ross, Electroweak and dark matter constraints on a Z-prime in models with a hidden valley, Nucl. Phys. B827, 256 (2010).

[100] G. Aad et al. (ATLAS Collaboration), Search for highmass dilepton resonances in pp collisions at $\sqrt{s}=8 \mathrm{TeV}$ with the ATLAS detector, Phys. Rev. D 90, 052005 (2014).

[101] V. Khachatryan et al. (CMS Collaboration), Search for physics beyond the standard model in dilepton mass spectra in proton-proton collisions at $\sqrt{s}=8 \mathrm{TeV}$, J. High Energy Phys. 04 (2015) 025.

[102] J. M. Cline, G. Dupuis, Z. Liu, and W. Xue, The windows for kinetically mixed Z'-mediated dark matter and the galactic center gamma ray excess, J. High Energy Phys. 08 (2014) 131.

[103] I. Hoenig, G. Samach, and D. Tucker-Smith, Searching for dilepton resonances below the $\mathrm{Z}$ mass at the LHC, Phys. Rev. D 90, 075016 (2014).

[104] J. P. Lees et al. (BABAR Collaboration), Search for a Dark Photon in $e^{+} e^{-}$Collisions at BABAR, Phys. Rev. Lett. 113, 201801 (2014).

[105] R. Aaij et al. (LHCb Collaboration), Search for Dark Photons Produced in $13 \mathrm{TeV} p p$ Collisions, Phys. Rev. Lett. 120, 061801 (2018).

[106] D. Tucker-Smith and N. Weiner, Inelastic dark matter, Phys. Rev. D 64, 043502 (2001). 
[107] D. Tucker-Smith and N. Weiner, The Status of inelastic dark matter, Phys. Rev. D 72, 063509 (2005).

[108] E. Izaguirre, G. Krnjaic, and B. Shuve, Discovering inelastic thermal-relic dark matter at colliders, Phys. Rev. D 93, 063523 (2016).

[109] R. Primulando, E. Salvioni, and Y. Tsai, The dark penguin shines light at colliders, J. High Energy Phys. 07 (2015) 031.

[110] A. Crivellin, U. Haisch, and A. Hibbs, LHC constraints on gauge boson couplings to dark matter, Phys. Rev. D 91, 074028 (2015).

[111] V. Khachatryan et al. (CMS Collaboration), Search for new phenomena in monophoton final states in proton-proton collisions at $\sqrt{s}=8 \mathrm{TeV}$, Phys. Lett. B 755, 102 (2016).

[112] G. Aad et al. (ATLAS Collaboration), Search for new phenomena in events with a photon and missing transverse momentum in $p p$ collisions at $\sqrt{s}=8 \mathrm{TeV}$ with the ATLAS detector, Phys. Rev. D 91, 012008 (2015); Erratum, 92, 059903 (2015).

[113] M. Aaboud et al. (ATLAS Collaboration), Search for dark matter at $\sqrt{s}=13 \mathrm{TeV}$ in final states containing an energetic photon and large missing transverse momentum with the ATLAS detector, Eur. Phys. J. C 77, 393 (2017).

[114] J. Liu, B. Shuve, N. Weiner, and I. Yavin, Looking for new charged states at the LHC: Signatures of Magnetic and Rayleigh Dark Matter, J. High Energy Phys. 07 (2013) 144.

[115] J. Preskill, M. B. Wise, and F. Wilczek, Cosmology of the invisible axion, Phys. Lett. 120B, 127 (1983).

[116] L. F. Abbott and P. Sikivie, A cosmological bound on the invisible axion, Phys. Lett. 120B, 133 (1983).

[117] M. Dine and W. Fischler, The not so harmless axion, Phys. Lett. 120B, 137 (1983).

[118] P. W. Graham, D. E. Kaplan, and S. Rajendran, Cosmological Relaxation of the Electroweak Scale, Phys. Rev. Lett. 115, 221801 (2015).

[119] J. Jaeckel and M. Spannowsky, Probing MeV to $90 \mathrm{GeV}$ axion-like particles with LEP and LHC, Phys. Lett. B 753, 482 (2016).

[120] G. Abbiendi et al. (OPAL Collaboration), Multiphoton production in $e^{+} e^{-}$collisions at $s^{* *}(1 / 2)=181-\mathrm{GeV}$ to 209-GeV, Eur. Phys. J. C 26, 331 (2003).

[121] G. Aad et al. (ATLAS Collaboration), Search for Scalar Diphoton Resonances in the Mass Range 65-600 GeV with the ATLAS Detector in $p p$ Collision Data at $\sqrt{s}=8 \mathrm{TeV}$, Phys. Rev. Lett. 113, 171801 (2014).

[122] G. Aad et al. (ATLAS Collaboration), Search for new phenomena in events with at least three photons collected in $p p$ collisions at $\sqrt{s}=8 \mathrm{TeV}$ with the ATLAS detector, Eur. Phys. J. C 76, 210 (2016).
[123] S. Knapen, T. Lin, H. K. Lou, and T. Melia, Searching for Axionlike Particles with Ultraperipheral Heavy-Ion Collisions, Phys. Rev. Lett. 118, 171801 (2017).

[124] The FCC-ee design study, http://tlep.web.cern.ch/.

[125] Circular electron positron collider, http://cepc.ihep.ac.cn/.

[126] J. Alwall, R. Frederix, S. Frixione, V. Hirschi, F. Maltoni, O. Mattelaer, H. S. Shao, T. Stelzer, P. Torrielli, and M. Zaro, The automated computation of tree-level and nextto-leading order differential cross sections, and their matching to parton shower simulations, J. High Energy Phys. 07 (2014) 079.

[127] Z. Liu, L.-T. Wang, and H. Zhang, Exotic Decays of the $125 \mathrm{GeV}$ Higgs Boson at Future $e^{+} e^{-}$Lepton Colliders, Chin. Phys. C 41, 063102 (2017).

[128] P. Azzi, C. Bernet, C. Botta, P. Janot, M. Klute, P. Lenzi, L. Malgeri, and M. Zanetti, Prospective studies for LEP3 with the CMS detector, arXiv:1208.1662.

[129] C. Patrignani et al. (Particle Data Group Collaboration), Review of particle physics, Chin. Phys. C 40, 100001 (2016).

[130] J. A. Dror, R. Lasenby, and M. Pospelov, New constraints on light vectors coupled to anomalous currents, Phys. Rev. Lett. 119, 141803 (2017).

[131] A. Ismail and A. Katz, Anomalous $Z^{\prime}$ and Diboson Resonances at the LHC, arXiv:1712.01840.

[132] S. Ipek, D. McKeen, and A. E. Nelson, A renormalizable model for the galactic center gamma ray excess from dark matter annihilation, Phys. Rev. D 90, 055021 (2014).

[133] T. Sjostrand, S. Mrenna, and P. Z. Skands, PYTHIA 6.4 physics and manual, J. High Energy Phys. 05 (2006) 026.

[134] J. de Favereau, C. Delaere, P. Demin, A. Giammanco, V. Lematre, A. Mertens, and M. Selvaggi (DELPHES 3 Collaboration), DELPHES 3, A modular framework for fast simulation of a generic collider experiment, J. High Energy Phys. 02 (2014) 057.

[135] S. Chatrchyan et al. (CMS Collaboration), Identification of b-quark jets with the CMS experiment, J. Instrum. 8, P04013 (2013).

[136] CMS Collaboration, Search for light vector resonances decaying to a quark pair produced in association with a jet in proton-proton collisions at $\sqrt{s}=13 \mathrm{TeV}$, Technical Report CMS-PAS-EXO-17-001, 2017.

[137] CMS Collaboration, Inclusive search for the standard model Higgs boson produced in pp collisions at $\sqrt{\mathrm{s}}=$ $13 \mathrm{TeV}$ using $\mathrm{H} \rightarrow \mathrm{b} \overline{\mathrm{b}}$ decays, Technical Report CMSPAS-HIG-17-010, 2017. 